International Journal of Bifurcation and Chaos, Vol. 26, No. 7 (2016) 1630016 (40 pages)

(C) The Author(s)

DOI: $10.1142 / \mathrm{S} 0218127416300160$

\title{
Quasi-Periodic Bifurcations of Higher-Dimensional Tori
}

\author{
Motomasa Komuro \\ Center for Fundamental Education, Teikyo University of Science, \\ 2525 Yatsusawa, Uenohara-shi, Yamanashi 409-0193, Japan \\ komuro@ntu.ac.jp \\ Kyohei Kamiyama* and Tetsuro Endo ${ }^{\dagger}$ \\ Department of Electronics and Bioinformatics, \\ Meiji University, 1-1-1 Higashi-Mita, Tama-ku, \\ Kawasaki-shi, Kanagawa 214-8571, Japan \\ *kamiyama@sat.t.u-tokyo.ac.jp \\ †endoh@meiji.ac.jp \\ Kazuyuki Aihara \\ Institute of Industrial Science, The University of Tokyo, \\ Komaba, Meguro-ku, Tokyo 153-8505, Japan \\ aihara@sat.t.u-tokyo.ac.jp
}

Received December 23, 2015

\begin{abstract}
We classify the local bifurcations of quasi-periodic $d$-dimensional tori in maps (abbr. MTd) and in flows (abbr. FTd) for $d \geq 1$. It is convenient to classify these bifurcations into normal bifurcations and resonance bifurcations. Normal bifurcations of MTd can be classified into four classes: namely, saddle-node, period doubling, double covering, and Neimark-Sacker bifurcations. Furthermore, normal bifurcations of FT $d$ can be classified into three classes: saddle-node, double covering, and Neimark-Sacker bifurcations. These bifurcations are determined by the type of the dominant Lyapunov bundle. Resonance bifurcations are well known as phase locking of quasi-periodic solutions. These bifurcations are classified into two classes for both MTd and FTd: namely, saddle-node cycle and heteroclinic cycle bifurcations of the $(d-1)$-dimensional tori. The former is reversible, while the latter is irreversible. In addition, we propose a method for analyzing higher-dimensional tori, which uses one-dimensional tori in sections (abbr. ST1) and zero-dimensional tori in sections (abbr. ST0). The bifurcations of ST1 can be classified into five classes: saddle-node, period doubling, component doubling, double covering, and NeimarkSacker bifurcations. The bifurcations of ST0 can be classified into four classes: saddle-node, period doubling, component doubling, and Neimark-Sacker bifurcations. Furthermore, we clarify the relationship between the bifurcations of ST1/ST0 and the bifurcations of MTd/FTd. We present examples of all of these bifurcations.
\end{abstract}

Keywords: Continuous-time dynamical system; discrete-time dynamical system; invariant closed curve; high-dimensional torus; quasi-periodic bifurcation; local bifurcation; normal bifurcation; resonance bifurcation; dominant Lyapunov bundle (DLB); one-dimensional torus in section; zero-dimensional torus in section.

\footnotetext{
This is an Open Access article published by World Scientific Publishing Company. It is distributed under the terms of the Creative Commons Attribution 4.0 (CC-BY) License. Further distribution of this work is permitted, provided the original work is properly cited.
} 


\section{Introduction}

Since quasi-periodic oscillations are ubiquitous in this real world full of various rhythms, bifurcations of quasi-periodic tori have been intensively studied (for example, see [Ruelle \& Takens, 1971; Broer et al., 1996, 2008]). The purpose of this paper is to investigate local bifurcations of quasi-periodic $d$-dimensional tori in maps (abbr. MTd) and in flows (abbr. FTd), for $d \geq 1$. The local bifurcations of quasi-periodic invariant tori can be classified into two classes; namely, resonance bifurcations and normal bifurcations (see Fig. 1). Resonance bifurcations are well known as phase locking of quasi-periodic solutions. For example, by resonance bifurcations, quasi-periodic invariant closed curves (abbr. ICC) in maps can be converted into stable periodic points and saddle periodic points, and quasi-periodic invariant two-tori in map can be converted into stable periodic ICCs and saddle periodic ICCs. We investigate such bifurcations in Sec. 4. On the other hand, normal bifurcations occur under the assumption that a resonance bifurcation does not occur, and include saddle-node (abbr. SN), period doubling (abbr. PD), double covering (abbr. DC) and Neimark-Sacker (abbr. NS) bifurcations. In our previous paper [Kamiyama et al., 2014], we classified the normal bifurcations of MT1 and FT2 by dominant Lyapunov bundles. In this paper, we extend those results to higher-dimensional tori. Furthermore, we investigate one-dimensional tori in sections (abbr. ST1) and zero-dimensional tori in sections (abbr. ST0), which constitute the numerical analysis of bifurcations of higher-dimensional tori.

The rest of this paper is structured as follows. In Sec. 2, we introduce technical terms, and state the normal bifurcation conjecture for quasiperiodic high-dimensional tori. In Sec. 3, we define $k$-dimensional tori in sections (abbr. ST $k$ ) for MTd and FTd, and consider bifurcations of STk especially for $k=0$ and 1. In Sec. 4, we investigate saddle-node cycle bifurcations and heteroclinic cycle bifurcations, which constitute resonance bifurcations. In Secs. 5 and 6 , we provide examples of possible bifurcations in both discrete-time and continuous-time dynamical systems. Our conclusions are presented in Sec. 7.

\section{Normal Bifurcations}

In order to avoid overlap with our previous paper [Kamiyama et al., 2014], we will focus our attention on new results. However, to make this article selfcontained, first we reiterate some necessary results.

\subsection{Lyapunov bundles of quasi-periodic invariant tori}

We denote continuous-time and discrete-time smooth dynamical systems by the terms flow and map, respectively. For simplicity, we will use $t$ to denote both continuous time $t \in \mathbb{R}$ in a flow $f^{t}$, and discrete time $t \in \mathbb{Z}$ in a map $f .^{1}$ A translational flow/map on the standard $d$-dimensional torus $\mathbb{T}^{d}=\mathbb{R}^{d} / \mathbb{Z}^{d}$, given by

$$
\begin{gathered}
x(t)=\Phi^{t}(x(0))=x(0)+\omega t, \\
x=\left(x_{1}, \ldots, x_{d}\right) \in \frac{\mathbb{R}^{d}}{\mathbb{Z}^{d}}, \quad \omega=\left(\omega_{1}, \ldots, \omega_{d}\right) \in \mathbb{R}^{d},
\end{gathered}
$$

is quasi-periodic if $\omega$ is rationally independent, meaning that $\langle k, \omega\rangle=\sum_{i=1}^{d} k_{i} \omega_{i} \neq 0$ holds for all $k=\left(k_{1}, \ldots, k_{d}\right) \in \mathbb{Z}^{d} \backslash\{0\}$.

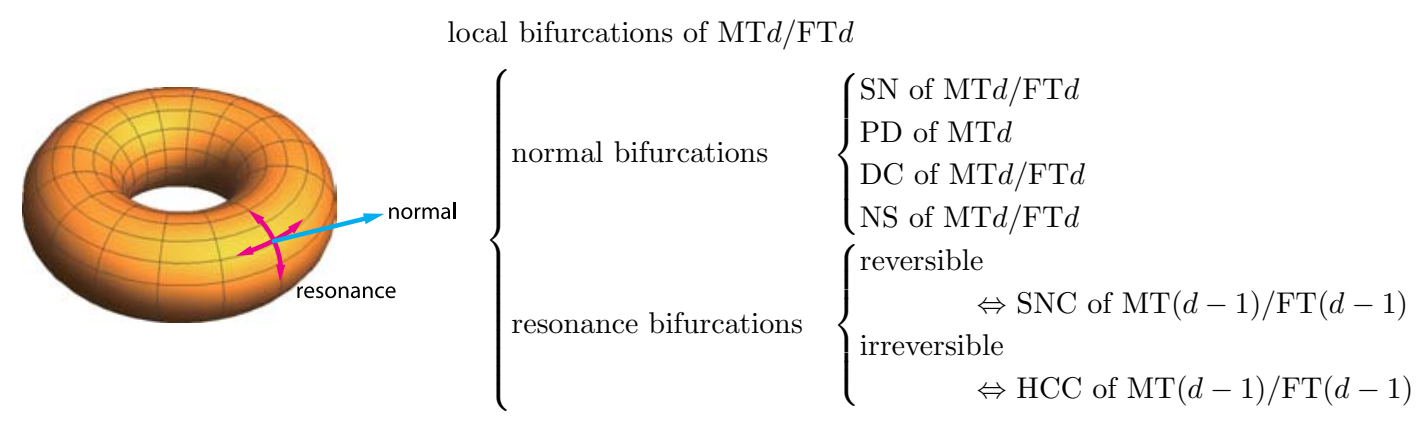

Fig. 1. Illustration of normal bifurcations and resonance bifurcations, where MTd/FTd means that either MTd or FTd.

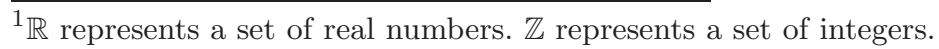


A flow-invariant subset that is topologically conjugate to a quasi-periodic translational flow on a standard $d$-dimensional torus is called a quasi-periodic $d$-dimensional torus in a flow. We denote such a subset by FTd. Similarly, a mapinvariant subset that is topologically conjugate to a quasi-periodic translational map on a standard $d$-dimensional torus is called a quasi-periodic $d$ dimensional torus in a map, and is denoted by MTd. We regard FT0 as an equilibrium point in a flow, and MT0 as a fixed point in a map. We also regard FT1 as a periodic orbit in a flow, and MT1 as a quasi-periodic invariant closed curve in a map.

Assume that a flow $f^{t}$ or map $f$ on $\mathbb{R}^{N}$ admits a quasi-periodic invariant $d$-dimensional torus $\Gamma$. We denote the tangent space of $\mathbb{R}^{N}$ at $x \in \mathbb{R}^{N}$ by $T_{x} \mathbb{R}^{N}$. According to the multiplicative ergodic theorem, proposed by Oseledets [Oseledets, 1968; Ruelle, 1979], for all points $x \in \Gamma$ except for measure zero subsets, there exist a real-number sequence $\lambda_{1}>\lambda_{2}>\cdots>\lambda_{m}$ and a direct sum decomposition of tangent space

$$
T_{x} \mathbb{R}^{N}=E_{1}(x) \oplus E_{2}(x) \oplus \cdots \oplus E_{m}(x),
$$

such that

$$
\begin{array}{r}
\lambda_{i}=\lim _{t \rightarrow \pm \infty} \frac{1}{t} \log \frac{\left\|d f^{t}(x) v\right\|}{\|v\|}, \quad v \in E_{i}(x) \backslash\{0\}, \\
1 \leq i \leq m(\leq N) .
\end{array}
$$

In Eq. (3), $d f^{t}(x)$ is the Jacobian matrix of $f^{t}$ at $x$.

For each $i(1 \leq i \leq m)$, the real number $\lambda_{i}$, the subspace $E_{i}(x)$, and the vector $v \in E_{i}(x)$ are called the Lyapunov exponent (abbr. LE), the Lyapunov eigenspace, and the Lyapunov vector, respectively.

The tangent bundle $T \mathbb{R}^{N}$ is the following set of all pairs $(x, v)$, where $x \in \mathbb{R}^{N}$ and $v \in T_{x} \mathbb{R}^{N}$ :

$$
T \mathbb{R}^{N}=\left\{(x, v): x \in \mathbb{R}^{N}, v \in T_{x} \mathbb{R}^{N}\right\} .
$$

Because each tangent space $T_{x} \mathbb{R}^{N}$ is homeomorphic to $\mathbb{R}^{N}, T \mathbb{R}^{N}$ is homeomorphic to the direct product space $\mathbb{R}^{N} \times \mathbb{R}^{N}$.

The restriction of $T \mathbb{R}^{N}$ to $\Gamma$ is given by

$$
T_{\Gamma} \mathbb{R}^{N}=\left\{(x, v): x \in \Gamma, v \in T_{x} \mathbb{R}^{N}\right\}
$$

and is called the tangent bundle on the base space $\Gamma$. For each $\lambda_{i}$, the Lyapunov bundle $\operatorname{LB}\left(\lambda_{i}\right)$ is defined by

$$
\operatorname{LB}\left(\lambda_{i}\right)=\left\{(x, v): x \in \Gamma, v \in E_{i}(x)\right\}
$$

and is a subbundle of $T_{\Gamma} \mathbb{R}^{N}$. In general, a Lyapunov bundle $\operatorname{LB}\left(\lambda_{i}\right)$ is locally (but not necessarily totally) homeomorphic to the direct product $\Gamma \times \mathbb{R}^{N}$. Essentially, the Lyapunov bundle collects the Lyapunov eigenspaces $E_{i}(x)$ for all $x \in \Gamma$. For FT1 (a periodic orbit in flow), the Lyapunov exponent, Lyapunov vector, and Lyapunov eigenspace coincide with the Floquet exponent, Floquet vector, and Floquet eigenspace, respectively. Therefore, the Lyapunov bundle in FT1 should be called a Floquet bundle. The Lyapunov bundle generalizes the Floquet bundle to $\mathrm{MT} d(d \geq 1)$ or FTd $(d \geq 2)$.

Given a map $f$, we define the tangent map $T f$ on $T \mathbb{R}^{N}$ by

$$
\begin{aligned}
T f & : \mathbb{R}^{N} \times T_{x} \mathbb{R}^{N} \ni(x, v) \\
& \mapsto(f(x), d f(x) v) \in \mathbb{R}^{N} \times T_{f(x)} \mathbb{R}^{N},
\end{aligned}
$$

where $d f(x)$, the Jacobian matrix of $f$ at $x$, is a linear map from $T_{x} \mathbb{R}^{N}$ to $T_{f(x)} \mathbb{R}^{N}$. Because $\Gamma$ is invariant, $T f$ is well defined on $T_{\Gamma} \mathbb{R}^{N}$. In addition, because the Jacobian matrix $d f(x)$ maps $E_{i}(x)$ to $E_{i}(f(x))$ for $x \in \Gamma$, the tangent map $T f$ is well defined on the Lyapunov bundle $\operatorname{LB}\left(\lambda_{i}\right)$ for each $i$.

Similarly, for a flow $f^{t}$ we define the tangent flow $T f^{t}$ on $T \mathbb{R}^{N}$ by

$$
\begin{aligned}
T f^{t} & : \mathbb{R}^{N} \times T_{x} \mathbb{R}^{N} \ni(x, v) \\
& \mapsto\left(f^{t}(x), d f^{t}(x) v\right) \in \mathbb{R}^{N} \times T_{f^{t}(x)} \mathbb{R}^{N},
\end{aligned}
$$

where $d f^{t}(x)$, the Jacobian matrix of $f^{t}$ at $x$, is a linear map from $T_{x} \mathbb{R}^{N}$ to $T_{f^{t}(x)} \mathbb{R}^{N}$. Because $\Gamma$ is invariant, $T f^{t}$ is well defined on $T_{\Gamma} \mathbb{R}^{N}$. Furthermore, because the Jacobian matrix $d f^{t}(x)$ maps $E_{i}(x)$ to $E_{i}\left(f^{t}(x)\right)$ for $x \in \Gamma$, the tangent flow $T f^{t}$ is well defined on the Lyapunov bundle $\operatorname{LB}\left(\lambda_{i}\right)$ for each $i$.

The dimension of $E_{i}(x)$ is called the multiplicity of $\lambda_{i}$, and is denoted by $M\left(\lambda_{i}\right)$. On a $d$ dimensional invariant torus, the multiplicity of zero LEs is $d$ with $d \geq 1$. For flows (excluding an equilibrium point), because the LE along the flow is always zero, the multiplicity of zero LEs is one or higher. The LE related to the bifurcation of the quasi-periodic invariant torus is the nonzero $\mathrm{LE}$ with magnitude closest to zero. We call this quantity the dominant Lyapunov exponent (abbr. DLE), and its associated Lyapunov bundle is called the dominant Lyapunov bundle (abbr. DLB).

LEs provide the rates of exponential divergence or convergence in the directions of the Lyapunov tangent vectors. These are generally defined at $x \in \Gamma$, excluding the zero-measure points on $\Gamma$. 
We assume that the DLE exists at all $x \in \Gamma$, and that the associated DLB is smooth with respect to $x \in \Gamma$, although these properties are not necessarily guaranteed.

If all nonzero LEs are negative, the MTd/FTd is stable. If some positive LEs exist, the MTd/FTd is unstable. We assume that the saddle MTd/FTd has only one positive LE, in this paper.

\subsection{Classification of Lyapunov bundles}

The vector bundle whose fibers are $\mathbb{R}^{k}$ is called an $\mathbb{R}^{k}$-bundle. For $k=1,2$, we will topologically classify $\mathbb{R}^{k}$-bundles on a $d$-dimensional torus $\mathbb{T}^{d}$ for $d \geq 1$.

First, we classify the $\mathbb{R}^{1}$-bundle on the onedimensional torus $\mathbb{T}^{1}=\mathbb{R}^{1} / \mathbb{Z}^{1}$. Because an $\mathbb{R}^{1}$ bundle on $\mathbb{T}^{1}$ is locally the direct product of $\mathbb{T}^{1}$ and $\mathbb{R}^{1}$, it is homeomorphic to either the annulus or the Möbius band. We classify the former and the latter as types $A$ and $M$, respectively. Type $A$ is orientable, while type $M$ is nonorientable.

Next, we investigate the $\mathbb{R}^{1}$-bundle on the twodimensional torus $\mathbb{T}^{2}=\mathbb{R}^{2} / \mathbb{Z}^{2}$. There exist two closed curves $\gamma_{1}$ and $\gamma_{2}$ on $\mathbb{T}^{2}$ that are components of a basis of the fundamental group of $\mathbb{T}^{2}$. Because the restriction of the $\mathbb{R}^{1}$-bundle to $\gamma_{i}$ for $i=1,2$ is an $\mathbb{R}^{1}$-bundle of the one-dimensional torus, it is classified as either type $A$ or type $M$. Hence, an $\mathbb{R}^{1}$-bundle on $\mathbb{T}^{2}$ can be formally described as type $A \times A, M \times A$, or $M \times M$. However, because type $M \times A$ is homeomorphic to type $M \times M$ (see Appendix of [Kamiyama et al., 2014]), an $\mathbb{R}^{1}$-bundle on $\mathbb{T}^{2}$ is classified as either type $A \times A$ or $M \times M$. The former is orientable, while the latter is nonorientable.

Similarly, we can classify the $\mathbb{R}^{1}$-bundle on the $d$-dimensional torus $\mathbb{T}^{d}=\mathbb{R}^{d} / \mathbb{Z}^{d}$. Indeed, there exist $d$ closed curves $\gamma_{1}, \ldots, \gamma_{d}$ on $\mathbb{T}^{d}$ that are components of a basis of the fundamental group of $\mathbb{T}^{n}$. Because the restriction of the $\mathbb{R}^{1}$-bundle to $\gamma_{i}$ for $i=1, \ldots, d$ is an $\mathbb{R}^{1}$-bundle of the onedimensional torus, it is classified as either type $A$ or type $M$. Hence, an $\mathbb{R}^{1}$-bundle on $\mathbb{T}^{d}$ can be formally described as of type $A \times \cdots \times A, M \times A \times \cdots \times A$, $M \times M \times A \times \cdots \times A, \ldots, M \times \cdots \times M \times A$, or $M \times \cdots \times M$.

By repeatedly applying the fact that $M \times A$ type $\mathbb{R}^{1}$-bundles on $\mathbb{T}^{2}$ are homeomorphic to $M \times M$ type $\mathbb{R}^{1}$-bundles on $\mathbb{T}^{2}$, we have that the types
$M \times A \times \cdots \times A, M \times M \times A \times \cdots \times A, \ldots$, and $M \times \cdots \times M \times A$ are all homeomorphic to the type $M \times \cdots \times M$. Hence, an $\mathbb{R}^{1}$-bundle on $\mathbb{T}^{d}$ is classified as type $A \times \cdots \times A$ or $M \times \cdots \times M$. The former is simply called type $A^{d}$, and is orientable, while the latter is called type $M^{d}$, and is nonorientable.

Finally, we investigate the $\mathbb{R}^{2}$-bundle on $\mathbb{T}^{d}$ for $d \geq 1$. Because it is homeomorphic to the direct product $\mathbb{T}^{d} \times \mathbb{R}^{2}$, it corresponds to the case of complex conjugate eigenvalues in the Floquet bundle. Hence, we name it as type $F^{d}$ (a $d$-dimensional focus type).

To classify DLBs, we consider the action of tangent flow/map. Assume that $\Gamma$ is an MTd of a map $f$ for $d \geq 1$. If $\Gamma$ has a DLE $\lambda$ with multiplicity $M(\lambda)=1$, then the DLB is an $\mathbb{R}^{1}$-bundle on $\mathbb{T}^{d}$. Therefore, it is classified as either type $A^{d}$ or type $M^{d}$. The tangent map $T f$ on a type $A^{d}$ DLB, which is orientable, is either orientation preserving or orientation reversing. In the orientation preserving case, we denote the pairing of the DLB and $T f$ by $A^{d+}$. In the orientation reversing case, the pairing of the DLB and $T f$ is denoted by $A^{d-}$. Because a type $M^{d}$ DLB is itself nonorientable, the orientation of the associated $T f$ cannot be determined. Hence, we classify the pairing of the DLB and $T f$ as type $M^{d}$. If $\Gamma$ has a DLE $\lambda$ with multiplicity $M(\lambda)=2$, then the associated DLB is an $\mathbb{R}^{2}$ bundle on $\mathbb{T}^{d}$, hence it is of type $F^{d}$. Consequently, in maps, the pairing of the DLB and $T f$ can be classified as one of four types: $A^{d+}, A^{d-}, M^{d}$, or $F^{d}$. Figure 2 depicts the DLBs of MT1. The red circles denote MT1. Figure 2(a) depicts the DLB of type $A^{+}$, which is denoted by a one-sided annulus. The tangent space is mapped to the same side by $T f$. Figure 2(b) depicts the DLB of type $A^{-}$, which is denoted by a two-sided annulus. The tangent space is mapped to the opposite side by $T f$. Figure 2(c) depicts the DLB of type $M$, which is denoted by a Möbius band. Figure 2(d) depicts the DLB of type $F$, which is denoted by a shape that resembles a test tube brush.

In the same manner, let us assume that $\Gamma$ is FT $d$ of a flow $f^{t}$ for $d \geq 1$. In maps, types $A^{d+}$ and $A^{d-}$ can be distinguished by the action of $T f$. However, in flows no such distinction is required, because the tangent flow $T f^{t}$ always preserves the orientation by continuous isotropic deformation of the identity map $T f^{0}$. Therefore, in flows the pairing of the DLB and $T f^{t}$ can be classified as one of three types: $A^{d+}, M^{d}$, or $F^{d}$. 
(b)

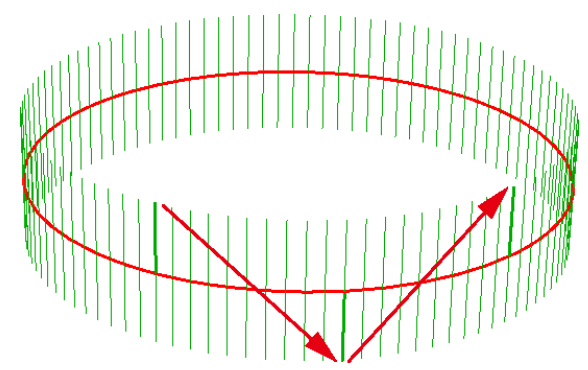

(c)
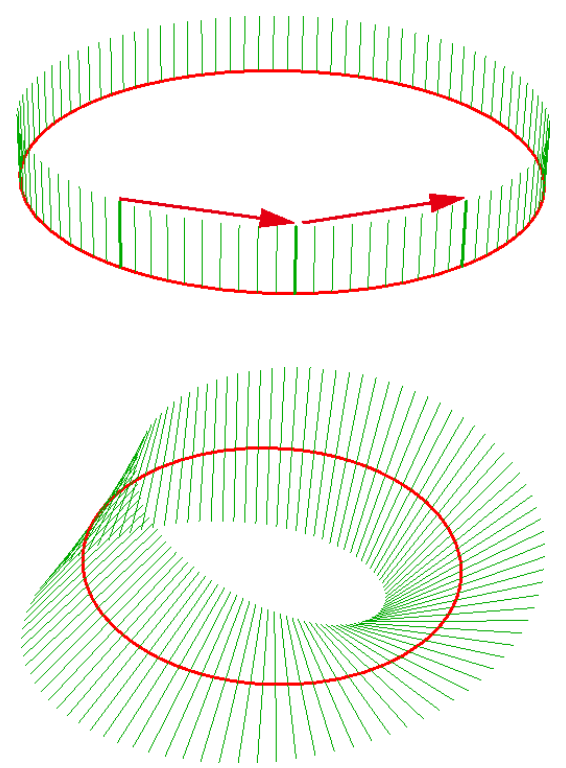

(a)

(c)

Fig. 2. Illustration of the DLBs of MT1. (a) Type $A^{+}$, (b) type $A^{-}$, (c) type $M$ and (d) type $F$. (d)

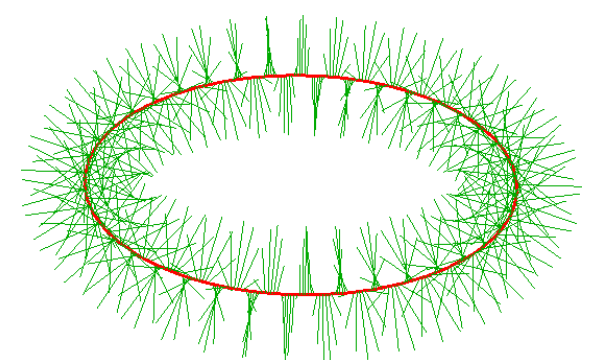

\subsection{Normal bifurcation conjecture}

Based on the above considerations, we present the following conjecture concerning the normal bifurcations of $\mathrm{MT} d(d \geq 1)$.

\section{MTdSN (saddle-node) bifurcation}

A saddle MTd and a stable MTd collide and vanish. This bifurcation occurs when the DLB is of type $A^{d+}$ prior to the bifurcation.

\section{MTdPD (period doubling) bifurcation}

A stable MTd becomes unstable, and stable twoperiodic MTds emerge in a neighborhood of the unstable MTd. ${ }^{2}$ This bifurcation occurs when the DLB is of type $A^{d-}$ prior to the bifurcation.

\section{MTdDC (double covering) bifurcation}

A stable MTd becomes unstable, and a stable double covering MTd emerges in a neighborhood of the unstable MTd. The MTdDC bifurcation doubles the covering degree of the MTd. This bifurcation occurs when the DLB is of type $M^{d}$ prior to the bifurcation.

\section{MTdNS (Neimark-Sacker) bifurcation}

A stable MTd becomes unstable, and a stable $\operatorname{MT}(d+1)$ emerges in a neighborhood of the unstable MTd. This bifurcation occurs when the DLB is of type $F^{d}$ prior to the bifurcation.
Similarly, we present the following conjecture concerning the normal bifurcations of FTd $(d \geq 1)$.

\section{FTdSN (saddle-node) bifurcation}

A saddle FTd and a stable FTd collide and vanish. This bifurcation occurs when the DLB is of type $A^{d+}$ prior to the bifurcation.

\section{FTdDC (double covering) bifurcation}

A stable FTd becomes unstable, and a stable double covering FTd emerges in a neighborhood of the unstable FTd. The FTdDC bifurcation doubles the covering degree of FTd. This bifurcation occurs when the DLB is of type $M^{d}$ prior to the bifurcation.

\section{FTdNS (Neimark-Sacker) bifurcation}

A stable FTd becomes unstable, and a stable $\mathrm{FT}(d+1)$ emerges in a neighborhood of the unstable FTd. This bifurcation occurs when the DLB is of type $F^{d}$ prior to the bifurcation.

\section{Bifurcations of Tori in Sections}

Here, we define one-dimensional tori in sections (abbr. ST1) and zero-dimensional tori in sections (abbr. ST0), which both play an important role in the numerical analysis of higher-dimensional tori.

Let $\Gamma \subset \mathbb{R}^{N}$ be MTd for a map $f$, or FTd for a flow $f^{t}$, for $d \geq 1$. For $k$ with $d \geq k \geq 0$, assume

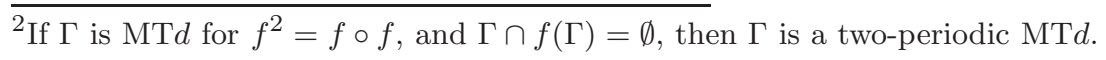


that $\Sigma$ is a $(N-d+k)$-dimensional surface, i.e. a codimension $(d-k)$ surface in $\mathbb{R}^{N}$. Then, the intersection $\gamma=\Gamma \cap \Sigma$ is $k$-dimensional in general. If the intersection $\gamma$ is a $k$-dimensional torus, then it is called a $k$-dimensional torus in a section (abbr. $\mathrm{ST} k)$ of $\mathrm{MT} d / \mathrm{FT} d$. Note that $\mathrm{ST} k$ is not invariant under the map $f$ or the flow $f^{t}$. The terms "stable", "unstable", "periodic", "Lyapunov bundle", or "bifurcation" are usually used for an invariant set of a dynamical system. Although STk is not an invariant set, it behaves in the same manner as an invariant set. Therefore, we propose here to apply these terms for STk in the following manner. STk of MTd/FTd will be called stable or unstable if the $\mathrm{MT} d / \mathrm{FT} d$ is stable or unstable, respectively. STk of MTd will be called $p$-periodic ${ }^{3}$ if the MT $d \Gamma$ is $p$-periodic; that is,

$$
\begin{aligned}
f^{p}(\Gamma)=\Gamma \quad & \text { and } \\
f^{i}(\Gamma) \cap \Gamma=\emptyset & (\forall i=1, \ldots, p-1) .
\end{aligned}
$$

If $\gamma$ is ST $k$ of the MTd/FTd, and $\lambda_{i}$ is a Lyapunov exponent of the $\mathrm{MT} d / \mathrm{FT} d$, then the restriction of the Lyapunov bundle $\operatorname{LB}\left(\lambda_{i}\right)$ to $\gamma$, defined by

$$
\operatorname{LB}_{\gamma}\left(\lambda_{i}\right)=\left\{(x, v): x \in \gamma, v \in E_{i}(x)\right\},
$$

is called the Lyapunov bundle of $\gamma$ for $\lambda_{i}$. The Lyapunov bundle of $\gamma$ for the dominant Lyapunov exponent is called the dominant Lyapunov bundle of $\gamma$ (abbr. DLB of $\gamma$ ). For the numerical calculation of STk, we take the following $\epsilon$ neighborhood of the codimension $(d-k)$ surface $\Sigma$

$$
\Sigma^{\epsilon}=\left\{x \in \mathbb{R}^{N}: \operatorname{dist}(\Sigma, x)<\epsilon\right\}
$$

and extract all the orbital points included in $\Sigma^{\epsilon}{ }^{4}$ We call this $\Sigma$ the codimension $(d-k)$-section, and $\Sigma^{\epsilon}$ the codimension $(d-k)$-slice with radius $\epsilon$. For the numerical calculation of the DLB of STk, we collect the DLBs of all the orbital points included in $\Sigma^{\epsilon}$.

In the following, we will consider only ST0 and $\mathrm{ST} 1$, because these are convenient for numerical analysis. It is worth noting that the DLB of an MTd can be classified into one of four types, $A^{d+}, A^{d-}$, $M^{d}$, or $F^{d}$, which correspond to $\mathrm{MT} d \mathrm{SN}, \mathrm{MT} d \mathrm{PD}$, $\mathrm{MT} d \mathrm{DC}$, and MTdNS bifurcations, respectively. Additionally, the DLB of FTd can be classified as one of three types, $A^{d+}, M^{d}$, or $F^{d}$, which correspond to FTdSN, FTdDC, and FTdNS bifurcations, respectively. ${ }^{5}$

We define the types of the DLB and the bifurcations for ST1 and ST0 (see Table 1). These definitions are described in detail in Secs. 3.1-3.4, using illustrations. A DLB of ST1 is described as type $A^{+}$ or $F$ if the corresponding DLB of the MTd/FTd is of type $A^{d+}$ or $F^{d}$, respectively. A DLB of ST1 is described as type $A^{-}$if the corresponding DLB of the MTd is of type $A^{d-}$. When the DLB of the $\mathrm{MT} d / \mathrm{FT} d$ is of type $M^{d}$, then the DLB of the ST1 is homeomorphic to either an annulus or a Möbius band. If it is homeomorphic to an annulus, then it is described as type $A^{*}$, and if it is homeomorphic to a Möbius band, then it is described as type $M$. A DLB of ST0 is described as type $A^{0+}, A^{0 *}$, or $F^{0}$ if the corresponding DLB of the MTd/FTd is of type $A^{d+}, M^{d}$, or $F^{d}$, respectively. Furthermore, a DLB of ST0 is described as type $A^{0-}$ if the corresponding DLB of the MTd is of type $A^{d-}$.

Table 1. The bifurcations and the types of DLB.

\begin{tabular}{|l||l|l|}
\hline $\mathrm{MT} d$ & $\mathrm{ST} 0$ & $\mathrm{ST} 1$ \\
\hline \hline $\mathrm{SN}, A^{d+}$ & $\mathrm{SN}, A^{0+}$ & $\mathrm{SN}, A^{+}$ \\
\hline $\mathrm{PD}, A^{d-}$ & $\mathrm{PD}, A^{0-}$ & $\mathrm{PD}, A^{-}$ \\
\hline \multirow{2}{*}{$\mathrm{DC}, M^{d}$} & \multirow{2}{*}{$\mathrm{CD}, A^{0 *}$} & $\mathrm{CD}, A^{*}$ \\
\cline { 3 - 3 } & & $\mathrm{DC}, M$ \\
\hline $\mathrm{NS}, F^{d}$ & $\mathrm{NS}, F^{0}$ & $\mathrm{NS}, F$ \\
\hline
\end{tabular}

\begin{tabular}{|l||l|l|}
\hline $\mathrm{FT} d$ & $\mathrm{ST} 0$ & $\mathrm{ST} 1$ \\
\hline \hline $\mathrm{SN}, A^{d+}$ & $\mathrm{SN}, A^{0+}$ & $\mathrm{SN}, A^{+}$ \\
\hline \multirow{2}{*}{$\mathrm{DC}, M^{d}$} & \multirow{2}{*}{$\mathrm{CD}, A^{0 *}$} & $\mathrm{CD}, A^{*}$ \\
\cline { 3 - 3 } & & $\mathrm{DC}, M$ \\
\hline $\mathrm{NS}, F^{d}$ & $\mathrm{NS}, F^{0}$ & $\mathrm{NS}, F$ \\
\hline
\end{tabular}

\footnotetext{
${ }^{3}$ Because the concept of the "period" of a quasi-periodic torus in a flow is not defined, the concept of a "period" is also not defined for STk of the FTd.

${ }^{4} \operatorname{dist}(A, x)$ is defined by $\inf \{\|y-x\|: y \in A\}$ for a closed set $A$ and a point $x$.

${ }^{5}$ The PD bifurcation is not defined for FTd.
} 
A bifurcation of ST1 is defined as ST1SN or ST1NS, if the bifurcation of the MTd/FTd is $\mathrm{MT} d \mathrm{SN} / \mathrm{FT} d \mathrm{SN}$ or MTdNS/FTdNS, respectively. A bifurcation of ST1 is defined as ST1PD, if the bifurcation of the MTd is MTdPD. When an MTdDC/FTdDC bifurcation occurs, the DLB of the ST1 is either of type $A^{*}$ or of type $M$. A bifurcation of ST1 is defined as ST1CD (the component doubling bifurcation of ST1), if the DLB of the ST1 is of type $A^{*}$. A bifurcation of ST1 is defined as ST1DC if the DLB of the ST1 is of type $M$. A bifurcation of ST0 is defined as ST0SN, ST0CD (the component doubling bifurcation of ST0), or STONS if the bifurcation of the $\mathrm{MT} d / \mathrm{FT} d$ is $\mathrm{MT} d \mathrm{SN} / \mathrm{FT} d \mathrm{SN}, \mathrm{MT} d \mathrm{DC} / \mathrm{FT} d \mathrm{DC}$, or $\mathrm{MT} d \mathrm{NS} / \mathrm{FT} d \mathrm{NS}$, respectively.

\section{1. $S N$ (saddle-node) bifurcations}

First, we explain saddle-node bifurcations of ST1 and ST0. Figure 3(a) illustrates the saddle-node bifurcation of MTd/FTd. In Fig. 3(a), the green rectangle $\Sigma_{1}$ denotes a codimension $(d-1)$ section in $\mathbb{R}^{N}$. The red torus $\Gamma_{s}$ and the blue torus $\Gamma_{u}$ denote a stable $\mathrm{MT} d / \mathrm{FT} d$ and a saddle $\mathrm{MT} d / \mathrm{FT} d$, respectively. The red circle $\gamma_{s}$ and the blue circle $\gamma_{u}$ represent examples of ST1 which are intersections of the red and blue tori, respectively, with $\Sigma_{1}$. The DLBs of $\gamma_{s}$ and $\gamma_{u}$ are of type $A^{+}$, and denoted by a one-sided annulus. By changing the parameter, the stable MTd/FTd and the saddle MTd/FTd collide and vanish. The notation $\Phi$ means the vanishing. Figure 3(b) depicts this development on $\Sigma_{1}$, which is shown by the green box. The blue rectangle $\Sigma_{2}$ denotes a codimension $d$ section. The red point $p_{s}$ and the blue point $p_{u}$ are examples of ST0 which are intersections of $\gamma_{s}$ and $\gamma_{u}$, respectively, with $\Sigma_{2}$. The DLBs of $\gamma_{s}$ and $\gamma_{u}$ are of type $A^{+}$, and denoted by a one-sided annulus. By changing the parameter, $\gamma_{s}$ and $\gamma_{u}$ collide and vanish. Figure 3(c) depicts this development on $\Sigma_{2}$. The stable ST0 $p_{s}$ and the saddle ST0 $p_{u}$ have DLBs of type $A^{0+}$, which are denoted by one-sided lines. By changing the parameter, $p_{s}$ and $p_{u}$ collide and vanish.

Based on the above considerations, we define saddle-node bifurcations of ST1 and ST0 as follows.

ST1SN: A saddle-node bifurcation of ST1 (abbr. ST1SN) is a bifurcation of ST1 where MTdSN/FTdSN occurs. A stable ST1 on a stable $\mathrm{MT} d / \mathrm{FT} d$ and a saddle ST1 on a saddle MTd/FTd collide and vanish. The type $A^{+}$DLB of the ST1 is

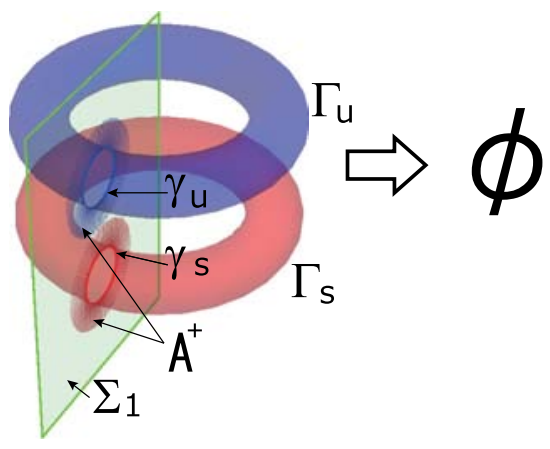

(a)

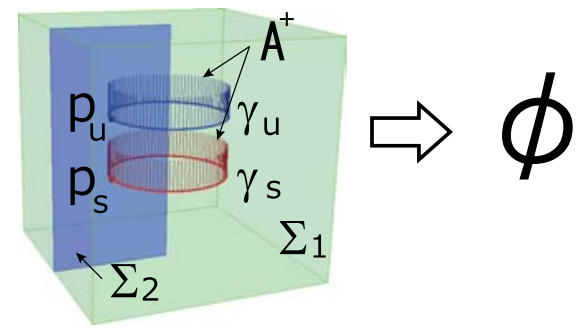

(b)
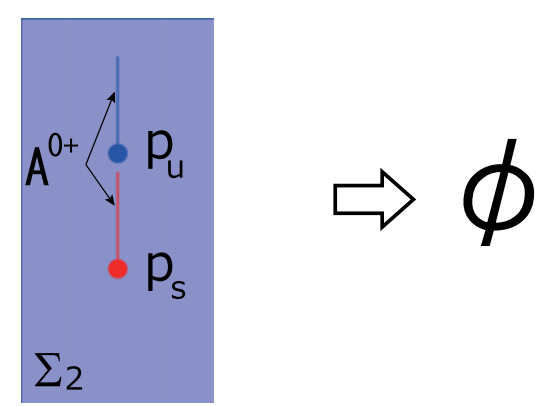

(c)

Fig. 3. Schematics of SN (saddle-node) bifurcations of $\mathrm{MTd} / \mathrm{FT} d$, ST1, and ST0. (a) MTdSN/FTdSN, (b) ST1SN and (c) STOSN.

homeomorphic to the annulus. In a numerical simulation, it appears on one side of the ST1.

ST0SN: A saddle-node bifurcation of ST0 (abbr. STOSN) is a bifurcation of ST0 where MTdSN/FTdSN occurs. A stable ST0 on a stable $\mathrm{MT} d / \mathrm{FT} d$ and a saddle ST0 on a saddle MTd/FTd collide and vanish. The type $A^{0+}$ DLB of the ST0 is homeomorphic to a line. In a numerical simulation, it appears on one side of the ST0.

\section{2. $P D$ (period doubling) bifurcations}

Next, we explain period doubling bifurcations of ST1 and ST0. Such bifurcations are only defined 


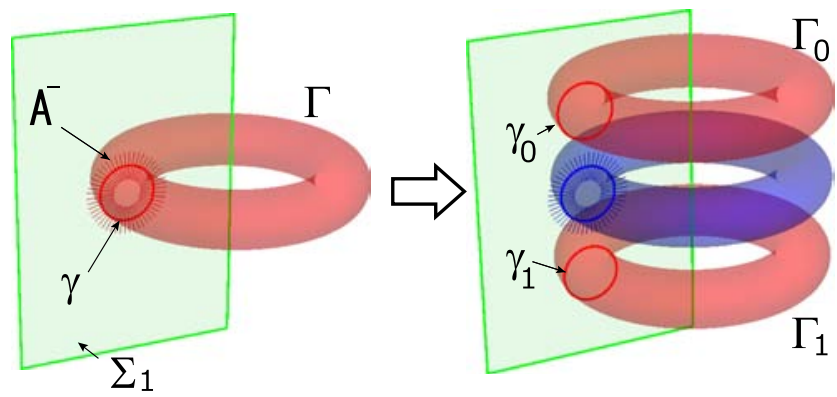

(a)

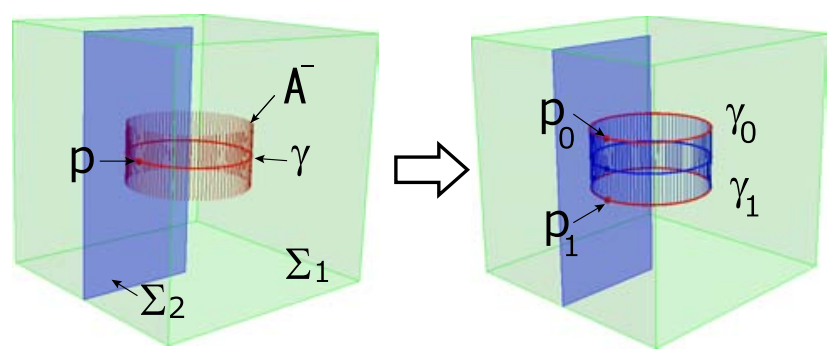

(b)
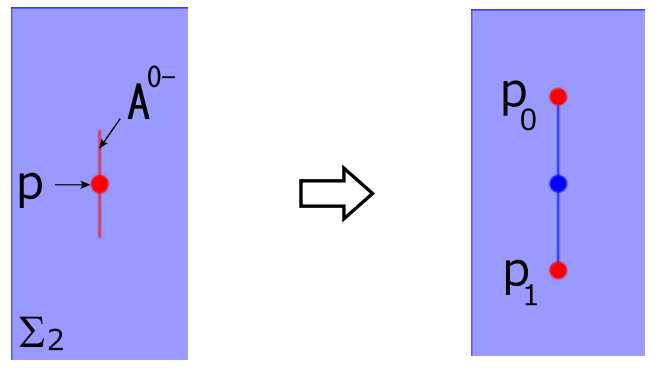

(c)

Fig. 4. Schematics of PD (period doubling) bifurcations of MTd, ST1, and ST0. (a) MTdPD, (b) ST1PD and (c) STOPD.

for MTd, because period doubling bifurcations are not defined for flows. Figure 4(a) depicts a period doubling bifurcation of MTd. In Fig. 4(a), the green rectangle $\Sigma_{1}$ denotes a codimension $(d-1)$ section in $\mathbb{R}^{N}$. The red torus $\Gamma$ denotes a stable MTd. The red circle $\gamma$ represents ST1 that is an intersection of $\Gamma$ with $\Sigma_{1}$. The DLB of $\gamma$ is of type $A^{-}$, which is denoted by a two-sided annulus. By changing the parameter, the stable MTd $\Gamma$ becomes an unstable MTd (the blue torus), and two stable twoperiodic MTds (the red tori) $\Gamma_{0}$ and $\Gamma_{1}$ appear in a neighborhood of the unstable MTd. Figure 4(b) illustrates this development on $\Sigma_{1}$, which is shown by the green box. The blue rectangle $\Sigma_{2}$ denotes a codimension $d$ section. The stable ST1 $\gamma$ has a DLB of type $A^{-}$, which is denoted by a two-sided annulus. By changing the parameter, $\gamma$ becomes an unstable ST1 (the blue circle), and a pair of stable two-periodic ST1s $\gamma_{0}$ and $\gamma_{1}$ (the red circles) appear in a neighborhood of the unstable ST1. Figure 4(c) illustrates this development on $\Sigma_{2}$. The stable ST0 $p$ has a DLB of type $A^{0-}$, which is denoted by a twosided line. By changing the parameter, $p$ becomes an unstable ST0 (the blue point), and a pair of stable two-periodic ST0s $p_{0}$ and $p_{1}$ (the red points) appear in a neighborhood of the unstable ST0.

Figure 5 depicts the bifurcation of the system in Fig. 4 under the twice-iterated map $f^{2}$. That is, when a PD bifurcation of $f$ occurs, a PF (pitchfork) bifurcation of $f^{2}$ occurs. ${ }^{6}$ In Fig. 5(a), the green rectangle $\Sigma_{1}$ denotes a codimension $(d-1)$ section in $\mathbb{R}^{N}$. The red torus $\Gamma$ denotes a stable MTd. The red circle $\gamma$ represents an ST1. Under $f^{2}$, the DLB of $\gamma$ is of type $A^{+}$, which is denoted by a one-sided annulus. By changing the parameter, the stable MTd $\Gamma$ becomes an unstable MTd (the blue torus), and two stable MTds appear in a neighborhood of the unstable MTd. In a numerical simulation of $f^{2}$, we only observe one stable MTd, which depends on the initial condition. The red and gray tori represent the observed and unobserved MTds, respectively. Figure 5(b) illustrates this development on $\Sigma_{1}$, which is shown by the green box. The blue rectangle $\Sigma_{2}$ denotes a codimension $d$ section. Under $f^{2}$, the stable ST1 $\gamma$ has a DLB of type $A^{+}$, which is denoted by a one-sided annulus. By changing the parameter, $\gamma$ becomes an unstable ST1 (the blue circle), and two stable ST1s appear in a neighborhood of the unstable ST1. In a numerical simulation of $f^{2}$, we only observe one stable ST1, which depends on the initial condition. The red and gray circles represent the observed and unobserved ST1s, respectively. Figure 5(c) illustrates this development on $\Sigma_{2}$. The stable ST0 $p$ has a DLB of type $A^{0+}$, which is denoted by a one-sided line. By changing the parameter, the stable ST0 $p$ becomes an unstable ST0 (the blue point), and two stable ST0s (the red and gray points) appear in a neighborhood of the unstable ST0. In a numerical simulation of $f^{2}$, we

\footnotetext{
${ }^{6}$ In the pitchfork bifurcation of MTd (abbr. MTdPF), a stable MTd becomes unstable, and two stable MTds appear in a neighborhood of the unstable MTd. A pitchfork bifurcation of ST0/ST1 (abbr. ST0PF/ST1PF) is a bifurcation of ST0/ST1 where MTdPF occurs.
} 


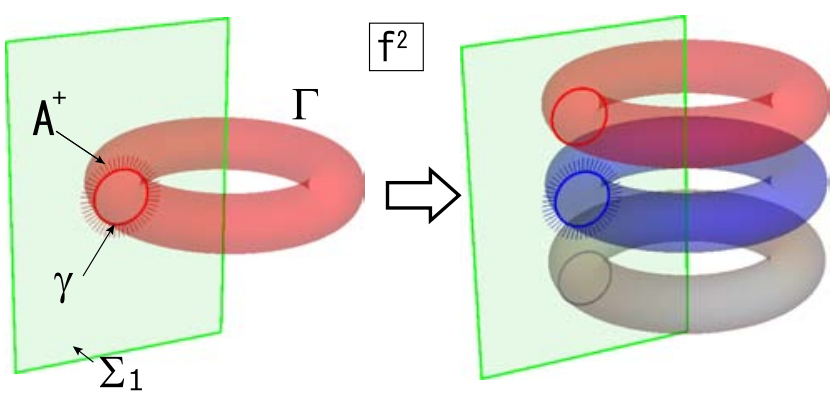

(a)

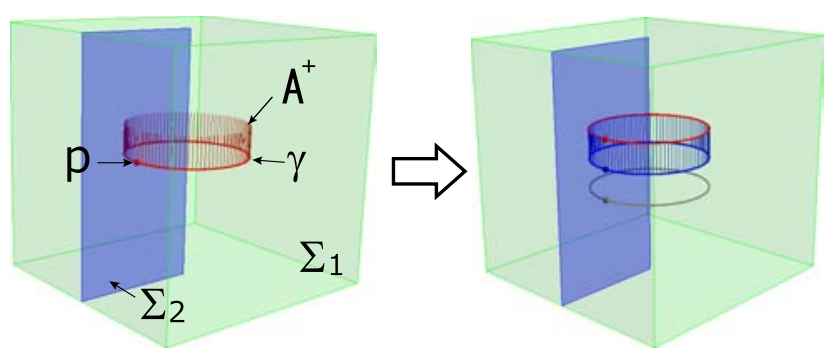

(b)
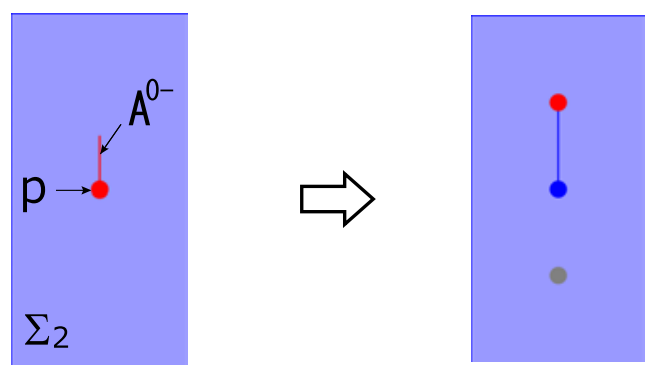

(c)

Fig. 5. Schematics of PF (pitchfork) bifurcations of MTd, ST1, and ST0 under $f^{2}$. (a) MTdPF, (b) ST1PF and (c) STOPF.

only observe one stable ST0, which depends on the initial conditions. The red and gray points represent the observed and unobserved ST0s, respectively.

Based on the above considerations, we define period doubling bifurcations of ST1 and ST0 as follows.

ST1PD: A period doubling bifurcation of ST1 (abbr. ST1PD) is a bifurcation of ST1 where MTdPD occurs. A stable ST1 becomes unstable, and stable two-periodic ST1s ${ }^{7}$ appear in a neighborhood of the unstable ST1. Prior to the bifurcation, the type $A^{-}$DLB of ST1 is homeomorphic to the two-sided annulus. In a numerical simulation, it appears on both sides of the ST1 under the onceiterated map. However, it appears only on one side of the ST1 under the twice-iterated map.

ST0PD: A period doubling bifurcation of ST0 (abbr. STOPD) is a bifurcation of ST0 where MTdPD occurs. A stable ST0 becomes unstable, and stable two-periodic ST0s ${ }^{8}$ appear in a neighborhood of the unstable ST0. Prior to the bifurcation, the type $A^{0-}$ DLB of ST0 is homeomorphic to the whole line. In a numerical simulation, it appears on both sides of the ST0 under the once-iterated map. However, it appears only on one side of the ST0 under the twice-iterated map.

\subsection{CD (component doubling) and DC (double covering) bifurcations}

Now, we explain component doubling and double covering bifurcations of ST1, and a component doubling bifurcation of ST0. Bifurcations of ST1 can be classified into two cases: namely, the CD (component doubling) bifurcation and the DC (double covering) bifurcation. Figure 6 illustrates a CD bifurcation of ST1, and Fig. 7 illustrates a DC bifurcation of ST1.

Figure 6 illustrates a CD bifurcation of $\mathrm{ST} 1$ in a DC bifurcation of MTd/FTd. In Fig. 6(a), the green rectangle $\Sigma_{1}$ denotes a codimension $(d-1)$ section in $\mathbb{R}^{N}$. The red torus $\Gamma$ denotes a stable $\mathrm{MT} d / \mathrm{FT} d$. The red circle $\gamma$ denotes an ST1 that is an intersection of $\Gamma$ with $\Sigma_{1}$. The DLB of $\gamma$ is of type $A^{*}$, which is denoted by a two-sided annulus. By changing the parameter, the stable $\mathrm{MT} d / \mathrm{FT} d \Gamma$ becomes an unstable MTd/FTd (the blue torus), and a stable double covering $\mathrm{MTd} / \mathrm{FT} d$ appears in a neighborhood of the unstable MTd/FTd. Figure 6(b) illustrates this development on $\Sigma_{1}$, which is shown by the green box. The blue rectangle $\Sigma_{2}$ denotes a codimension $d$ section. The stable ST1 $\gamma$ has a DLB of type $A^{*}$, which is denoted by a two-sided annulus. By changing the parameter, the stable ST1 $\gamma$ becomes an unstable ST1 (the blue circle), and two stable ST1s (the two red circles) appear in a neighborhood of the unstable ST1. We call this behavior a component doubling bifurcation of ST1 (abbr. ST1CD). For the case of a map, a PD bifurcation

\footnotetext{
${ }_{7}^{7}$ In a numerical simulation, two ST1s appear in the once-iterated map, however, one ST1 appears in the twice-iterated map.

${ }^{8}$ In a numerical simulation, two ST0s appear in the once-iterated map, however, one ST0 appears in the twice-iterated map.
} 

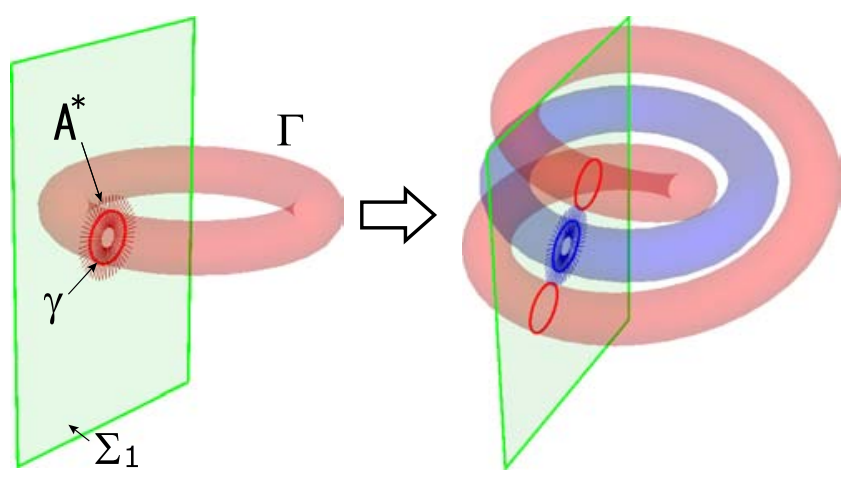

(a)
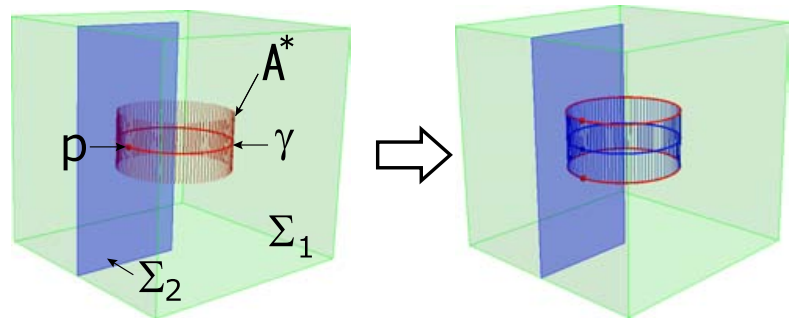

(b)
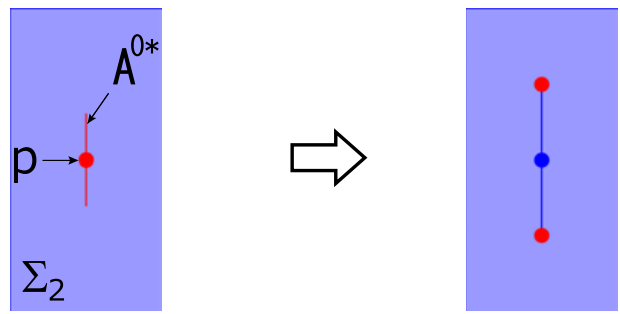

(c)

Fig. 6. Schematics of a DC (double covering) bifurcation of $\mathrm{MTd} / \mathrm{FT} d$, and CD (component doubling) bifurcations of ST1 and ST0. (a) MTdDC/FTdDC, (b) ST1CD and (c) ST0CD.

of ST1 becomes a PF bifurcation under the twiceiterated map. However, a CD bifurcation of ST1 is also a CD bifurcation under the twice-iterated map. Through this difference, we can distinguish ST1CD from ST1PD. Figure 6(c) illustrates this development on $\Sigma_{2}$. The stable ST0 $p$ has a DLB of type $A^{0 *}$, which is denoted by a two-sided line. By changing the parameter, $p$ becomes an unstable ST0 (the blue point), and two stable ST0s (the red points) appear in a neighborhood of the unstable ST0. For the case of a map, a PD bifurcation of ST0 becomes a PF bifurcation under the twiceiterated map. However, a CD bifurcation of ST0 is also a CD bifurcation under the twice-iterated map.
Through this difference, we can distinguish ST0CD from STOPD.

Figure 7 illustrates a DC bifurcation of ST1 in a DC bifurcation of MTd/FTd. In Fig. 7(a), the green rectangle $\Sigma_{1}$ denotes a codimension $(d-1)$ section in $\mathbb{R}^{N}$. The red torus $\Gamma$ denotes a stable MTd/FTd. The red circle $\gamma$ denotes ST1 that is an intersection of $\Gamma$ with $\Sigma_{1}$. The DLB of $\gamma$ is of type $M$, which is denoted by a Möbius band. By changing the parameter, the stable $\mathrm{MT} d / \mathrm{FT} d \Gamma$ becomes an unstable $\mathrm{MT} d / \mathrm{FT} d$ (the blue torus), and a stable double covering MTd/FTd appears in a neighborhood of the unstable MTd/FTd. Figure 7(b) illustrates this development on $\Sigma_{1}$, which is shown by the green box. The blue rectangle $\Sigma_{2}$ denotes a codimension $d$ section. The stable ST1 $\gamma$ has a DLB of

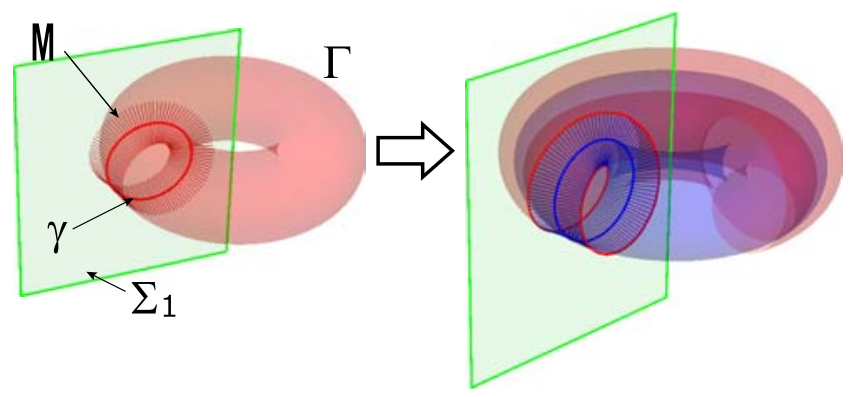

(a)
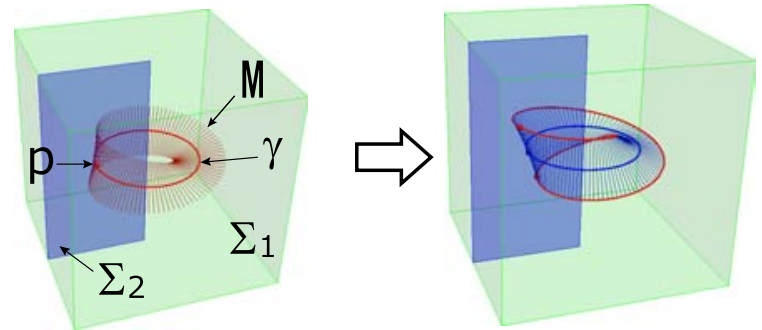

(b)
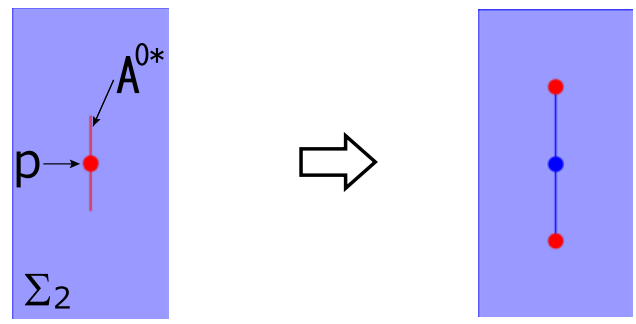

(c)

Fig. 7. Schematics of DC (double covering) bifurcations of MTd/FTd and ST1, and a CD (component doubling) bifurcation of ST0. (a) MTdDC/FTdDC, (b) ST1DC and (c) ST0CD. 
type $M$, which is denoted by a Möbius band. By changing the parameter, the stable ST1 $\gamma$ becomes an unstable ST1 (the blue circle), and a stable double covering ST1 appears in a neighborhood of the unstable ST1. We call this behavior a double covering bifurcation of ST1 (abbr. ST1DC). Figure 7(c) illustrates this development on $\Sigma_{2}$. The stable ST0 $p$ has a DLB of type $A^{0 *}$, which is denoted by a twosided line. By changing the parameter, the stable ST0 $p$ becomes an unstable ST0 (the blue point), and two stable ST0s (the red points) appear in a neighborhood of the unstable ST0.

Based on the above considerations, we define the DC (double covering) and CD (component doubling) bifurcations of ST1, and the CD (component doubling) bifurcations of ST0, as follows.

ST1DC: The double covering bifurcation of ST1 (abbr. ST1DC) is a bifurcation of ST1 where $\mathrm{MT} d \mathrm{DC} / \mathrm{FT} d \mathrm{DC}$ occurs, and the DLB of ST1 is of type $M$. A stable ST1 on a stable MTd/FTd becomes unstable, and a stable double covering ST1 appears in a neighborhood of the unstable ST1. A type $M$ DLB of ST1 is homeomorphic to a Möbius band.

ST1CD: The component doubling bifurcation of ST1 (abbr. ST1CD) is a bifurcation of ST1 where $\mathrm{MT} d \mathrm{DC} / \mathrm{FT} d \mathrm{DC}$ occurs, and the DLB of ST1 is of type $A^{*}$. A stable $\mathrm{ST} 1$ on a stable $\mathrm{MT} d / \mathrm{FT} d$ becomes unstable, and two stable ST1s appear in a neighborhood of the unstable ST1. A type $A^{*}$ DLB of ST1 is homeomorphic to an annulus. In a numerical simulation for MTd, it appears on both sides of the ST1 in both the once-iterated and twice-iterated maps. Through this difference, we can distinguish ST1CD from ST1PD.

ST0CD: The component doubling bifurcation of ST0 (abbr. ST0CD) is a bifurcation of ST0 where $\mathrm{MT} d \mathrm{DC} / \mathrm{FT} d \mathrm{DC}$ occurs, and the DLB of ST0 is of type $A^{0 *}$. A stable ST0 becomes unstable, and two stable ST0s appear in a neighborhood of the unstable ST0. A type $A^{0 *}$ DLB of ST0 is homeomorphic to the whole line. In a numerical simulation for MTd, it appears on both sides of the ST0 in both the once-iterated and twice-iterated maps. Through this difference, we can distinguish ST0CD from STOPD for an MTd.

\subsection{NS (Neimark-Sacker) bifurcations}

Next, we explain NS (Neimark-Sacker) bifurcations of ST1 and ST0. Figure 8(a) illustrates an NS bifurcation of MTd/FTd. In Fig. 8(a), the green rectangle $\Sigma_{1}$ denotes a codimension $(d-1)$ section in $\mathbb{R}^{N}$. The red torus $\Gamma$ is a stable MTd/FTd. The red circle $\gamma$ denotes ST1 that is an intersection of $\Gamma$ with $\Sigma_{1}$. The DLB of $\gamma$ is of type $F$, which is denoted by a shape resembling a test tube brush. By changing the parameter, the stable MTd/FT $\Gamma$ becomes an unstable $\mathrm{MT} d / \mathrm{FT} d$ (the blue torus), and a stable $\operatorname{MT}(d+1) / \mathrm{FT}(d+1)$ (the red torus) appears in a neighborhood of the unstable $\mathrm{MT} d / \mathrm{FT} d .^{9}$
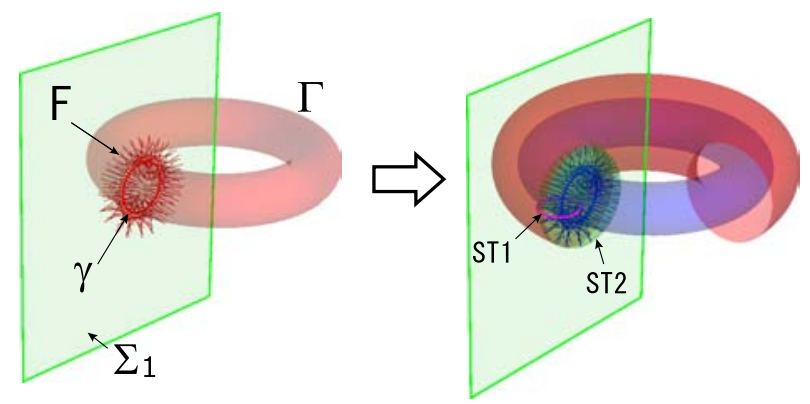

(a)
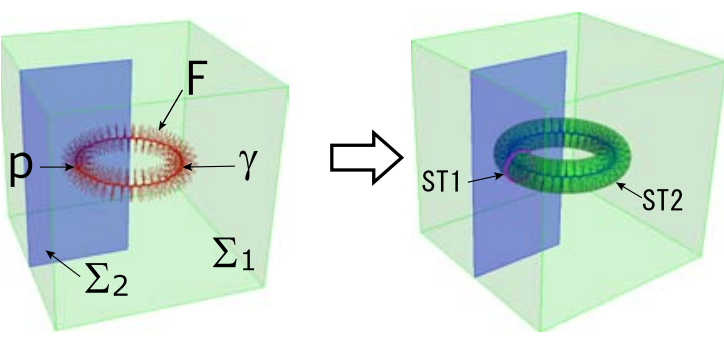

(b)
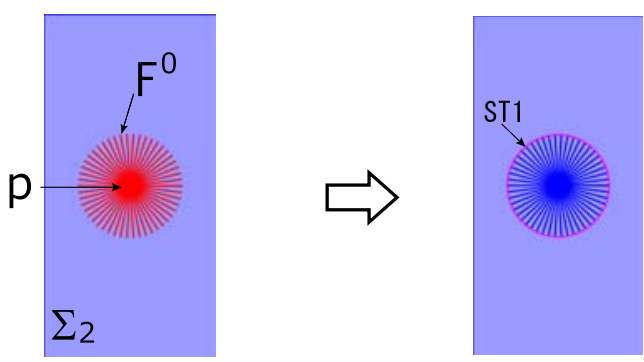

(c)

Fig. 8. Schematics of NS (Neimark-Sacker) bifurcations of MTd/FTd, ST1 and ST0. (a) MTdNS/FTdNS, (b) ST1NS and (c) STONS.

\footnotetext{
${ }^{9} \mathrm{~A}$ two-dimensional torus is used for representing a $(d+1)$-dimensional torus in Fig. 8 , since we cannot draw the threedimensional torus.
} 
Figure 8(b) illustrates this development on $\Sigma_{1}$ which is shown by the green box. The blue rectangle $\Sigma_{2}$ denotes a codimension $d$ section. The stable ST1 $\gamma$ has a DLB of type $F$. By changing the parameter, the stable ST1 $\gamma$ becomes an unstable ST1 (the blue circle), and a stable ST2 (the green torus) appears in a neighborhood of the unstable ST1. We call this behavior a Neimark-Sacker bifurcation of ST1 (abbr. ST1NS). Figure 8(c) illustrates this development on $\Sigma_{2}$. The stable ST0 $p$ has a DLB of type $F^{0}$, which is denoted by a disk with radial lines. By changing the parameter, the stable ST0 $p$ becomes an unstable ST0 (the blue point), and a stable ST1 (the magenta circle) appears in a neighborhood of the unstable ST0. We call this behavior a NeimarkSacker bifurcation of ST0 (abbr. STONS).

Based on the above considerations, we define the Neimark-Sacker bifurcations of ST1 and ST0 as follows.

ST1NS: The Neimark-Sacker bifurcation of ST1 (abbr. ST1NS) is a bifurcation of ST1 where MTdNS/FTdNS occurs. Then, the DLB of ST1 is of type $F$. A stable ST1 becomes unstable, and a stable ST2 (i.e. a two-dimensional torus in a section) appears in a neighborhood of the unstable ST1. In a numerical simulation, a type $F$ DLB of ST1 has a shape resembling a test tube brush.

ST0NS: The Neimark-Sacker bifurcation of ST0 (abbr. STONS) is a bifurcation of ST0 where MTdNS/FTdNS occurs. Then, the DLB of ST0 is of type $F^{0}$. A stable ST0 becomes unstable, and a stable ST1 appears in a neighborhood of the unstable ST0. In a numerical simulation, a type $F^{0}$ DLB of ST0 has a shape of a disk with radial lines.

\subsection{Normal bifurcation conjectures using ST1 and STO}

Based on the above considerations, we present the following conjectures concerning normal bifurcations of MTd and FTd using ST1 and ST0.

\section{MTdSN (saddle-node) bifurcation}

MTdSN occurs if either ST1SN or STOSN is observed.

\section{MTdPD (period doubling) bifurcation}

MTdPD occurs if either ST1PD or ST0PD is observed.

\section{MTdDC (double covering) bifurcation}

MTdDC occurs if ST1DC, ST1CD, or ST0CD is observed.

\section{MTdNS (Neimark-Sacker) bifurcation}

MTdNS occurs if either ST1NS or ST0NS is observed.

\section{FTdSN (saddle-node) bifurcation}

FTdSN occurs if either ST1SN or ST0SN is observed.

\section{FT $d$ DC (double covering) bifurcation}

FTdDC occurs if ST1DC, ST1CD, or ST0CD is observed.

\section{FT $d$ NS (Neimark-Sacker) bifurcation}

FTdNS occurs if either ST1NS or ST0NS is observed.

The validity of these conjectures will be illustrated using numerical examples, in Secs. 5 and 6.

Remark. The above method for employing ST1/ST0 is called the ST-method. The Poincaré sections and Poincaré return maps are well known conventional methods for studying bifurcations of flows. The ST-method is superior to such conventional methods in the following two points.

First, the codimension one section of the STmethod does not have to be transversal to the flow, but the Poincaré section must be transversal to the flow to define the Poincaré return map. For example, Fig. 9 presents ST1 in FT2. The green plane $\Sigma$ denotes a codimension one section, the blue circle denotes ST1, that is an intersection of $\Sigma$ with FT2.

Figure 9 (a) shows a flow that is transversal to $\Sigma$. In this case, we can define a Poincaré return map, and a bifurcation of FT2 can be reduced to a bifurcation of MT1 for the map. However, Fig. 9(b) shows a flow that is not transversal to $\Sigma$; the flow is tangent to $\Sigma$ at some points on ST1. In this case, we cannot define any return maps on $\Sigma$, and we cannot reduce a bifurcation of FT2 to a bifurcation of any MT1. This is true in general for FTd $(d \geq 2)$. That is, if we cannot take any codimension one section that is transversal to the flow, a bifurcation of FTd cannot be reduced to a bifurcation of any $\operatorname{MT}(d-1)$. On the other hand, the ST-method can be used in any case, whether $\Sigma$ is transversal to the flow or not. 


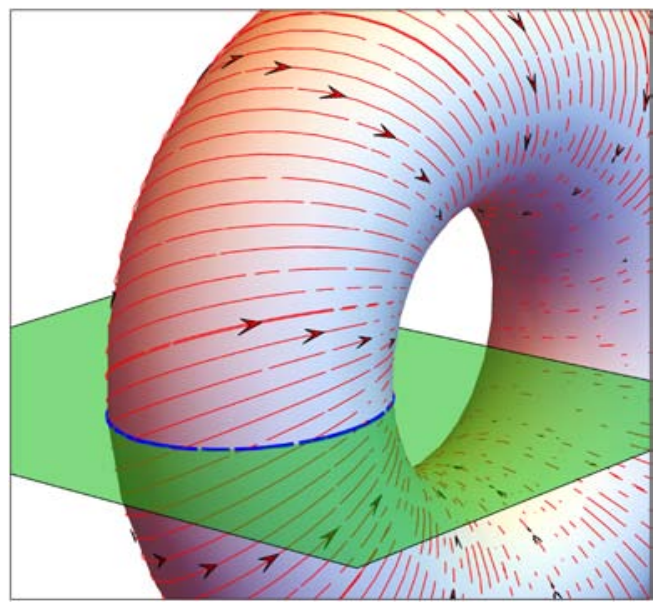

(a)

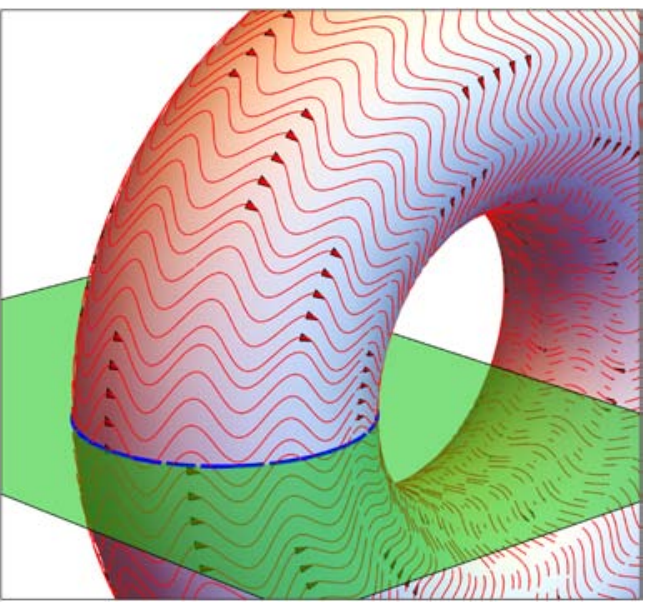

(b)

Fig. 9. Illustration of ST1 in FT2. The green plane and blue circle denote a codimension one section and ST1, respectively. (a) A transversal flow for ST1 and (b) a nontransversal flow for ST1.

Second, the ST-method can reduce an $n$-dimensional problem to a one-dimensional or a zero-dimensional problem, but the Poincaré method only reduces an $n$-dimensional problem of a flow to an $(n-1)$-dimensional problem of a map. For example, we can reduce a four-dimensional bifurcation problem of FT4 to a one-dimensional bifurcation problem of ST1, and further to a zero-dimensional bifurcation problem of ST0. However, the Poincaré method only reduces a four-dimensional bifurcation problem of FT4 to a three-dimensional bifurcation problem of MT3, which is difficult to analyze in general.

\section{Saddle-Node Cycle and Heteroclinic Cycle Bifurcations}

In this section, we investigate resonance bifurcations of $\operatorname{MT}(d+1) / \mathrm{FT}(d+1)$, which are classified into two classes: saddle-node cycle (MTdSNC/ FTdSNC) and heteroclinic ${ }^{10}$ cycle (MTdHCC/ FT $d$ HCC) bifurcations of MTd/FTd. Namely, the resonance bifurcations of $\mathrm{MT}(d+1) / \mathrm{FT}(d+1)$ are represented by global bifurcations of $d$-dimensional tori that occur by heteroclinic connections of stable and unstable sets. The former is reversible, while the latter is irreversible. In this section, we assume that the saddle MTd/FTd $\Gamma$ has only one positive
LE. Then, since the multiplicity of zero LEs is $d$, the number of negative LEs is $N-d-1$.

Let $U \subset \mathbb{R}^{N}$ be a small neighborhood of $\Gamma$. Then, we define the local stable set of $\Gamma$ by

$$
W_{\text {loc }}^{s}(\Gamma)=\left\{x \in U: \lim _{t \rightarrow \infty} \operatorname{dist}\left(\Gamma, f^{t}(x)\right)=0\right\}
$$

and the stable set of $\Gamma$ by

$$
W^{s}(\Gamma)=\bigcup_{t>0} f^{-t}\left(W_{\mathrm{loc}}^{s}(\Gamma)\right) .
$$

Similarly, we define the local unstable set of $\Gamma$ by

$$
W_{\text {loc }}^{u}(\Gamma)=\left\{x \in U: \lim _{t \rightarrow \infty} \operatorname{dist}\left(\Gamma, f^{-t}(x)\right)=0\right\}
$$

and the unstable set of $\Gamma$ by

$$
W^{u}(\Gamma)=\bigcup_{t>0} f^{t}\left(W_{\text {loc }}^{u}(\Gamma)\right) .
$$

Since the number of positive or zero LEs is $d+1$, $W^{u}(\Gamma)$ is $(d+1)$-dimensional. And, since the number of negative or zero LEs is $N-1, W^{s}(\Gamma)$ is $(N-1)$-dimensional. Hence $W^{s}(\Gamma)$ separates $\mathbb{R}^{N}$ into two parts.

\subsection{Saddle-node cycle bifurcations}

Figure 10(a) illustrates a saddle-node cycle bifurcation of MT0 (abbr. MTOSNC) ${ }^{11}$ in $\mathbb{R}^{2}$, while

\footnotetext{
${ }^{10}$ We use the term "heteroclinic" including the case of "homoclinic", because "homoclinic" can be considered as a special case of "heteroclinic".

${ }^{11}$ This bifurcation is called a saddle-node homoclinic/heteroclinic bifurcation in [Kuznetsov, 2004]. The inverse of this bifurcation is well known as phase locking of MT1 without hysteresis.
} 


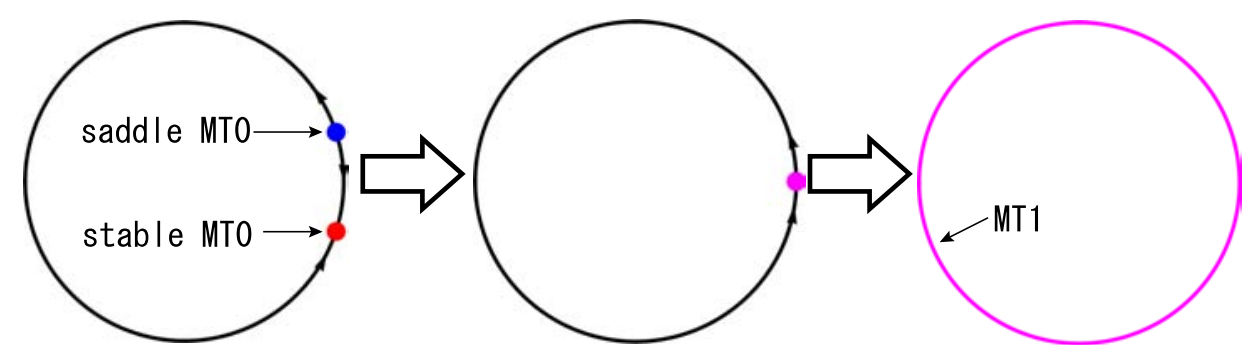

(a)

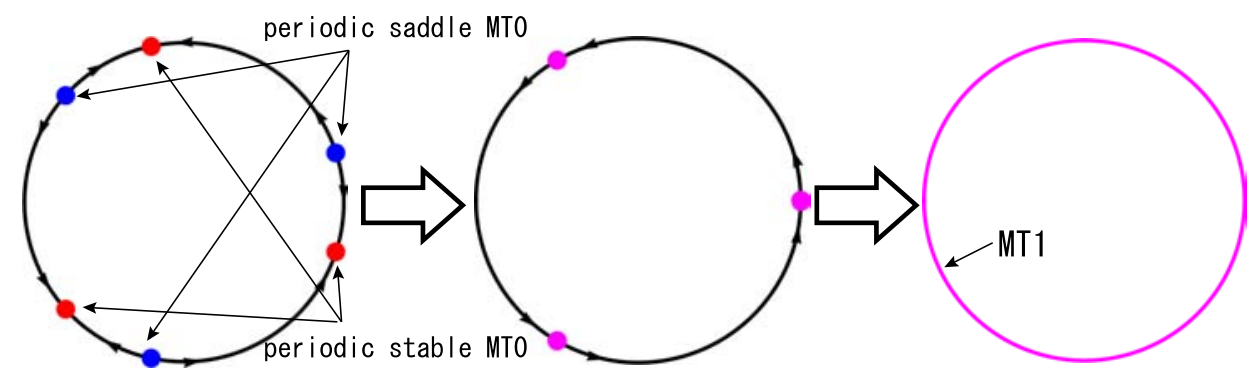

(b)

Fig. 10. Schematics of MTOSNC bifurcations. (a) MTOSNC for a simple MT0 and (b) MT0SNC for a periodic MT0.

Fig. 10(b) illustrates a saddle-node cycle bifurcation of a periodic MT0 in $\mathbb{R}^{2}$. The unstable sets of saddle MT0s form a circle, and stable MT0s and unstable MT0s that are alternately aligned on the circle bifurcate into MT1 by a saddle-node bifurcation when a parameter changes.

We extend this concept to saddle-node cycle bifurcations of MTd (abbr. MTdSNC) and FTd (abbr. FTdSNC); that is, unstable sets of saddle $\mathrm{MT} d \mathrm{~s} / \mathrm{FT} d \mathrm{~s}$ form a $(d+1)$-dimensional torus, and stable MTds/FTds and saddle MTds/FTds that are alternately aligned on a circle bifurcate into $\mathrm{MT}(d+1) / \mathrm{FT}(d+1)$ by an MTdSN/FTdSN bifurcation when a parameter changes.

Figure 11 illustrates MTdSNC/FTdSNC in $\mathbb{R}^{N}$. The red circles and the blue circles denote stable MTds/FTds and saddle MTds/FTds, respectively. The green rectangle denotes a codimension $d$ section $\Sigma$. The red points and the blue points denote stable ST0s and saddle ST0s on $\Sigma$, respectively. The magenta tori and circles denote $\mathrm{MT}(d+$ 1) and ST1, respectively. Figure 11(a) illustrates MTdSNC/FTdSNC for a simple MTd/FTd. Figure 11(b) illustrates MTdSNC for a periodic MTd. Figure 11(c) illustrates MTdSNC/FTdSNC for a winding MTd/FTd.

For a numerical analysis of MTdSNC/FTdSNC, we observe bifurcations of ST0s that are intersection of a codimension $d$ section $\Sigma$ with $\mathrm{MT} d / \mathrm{FT} d$.
On $\Sigma$, stable ST0s and saddle ST0s that are alternately aligned on a circle bifurcate into ST1 by a STOSN when a parameter changes. We call this phenomenon a saddle-node cycle bifurcation of ST0 (abbr. STOSNC).

\subsection{Heteroclinic cycle bifurcations}

Figure 12(a) illustrates a homoclinic cycle bifurcation of MT0 (abbr. MTOHCC), where a saddle fixed point of a map on a two-dimensional space has a one-dimensional unstable set $W^{u}$ and a onedimensional stable set $W^{s}$ that form a circle by a homoclinic connection, which is called a homoclinic circle. We assume that the product of the two eigenvalues of saddle MT0 is greater than 1, and there exists a repelling MT0 with complex eigenvalues inside the homoclinic circle. When a parameter changes, $W^{u}$ comes close to and touches $W^{s}$ from the outside, then intersects transversally with $W^{s}$. Further, $W^{u}$ touches $W^{s}$ from the inside, then separates from $W^{s}$. At a parameter value in an interval that $W^{u}$ intersects transversally with $W^{s}$, tangled MT1 is created, because a part of $W^{u}$ inside $W^{s}$ converges to MT1. The saddle fixed point remains through the bifurcation.

Similarly, Fig. 12(b) illustrates a heteroclinic cycle bifurcation of a periodic MT0 (abbr. MTOHCC), where saddle periodic points of a map 


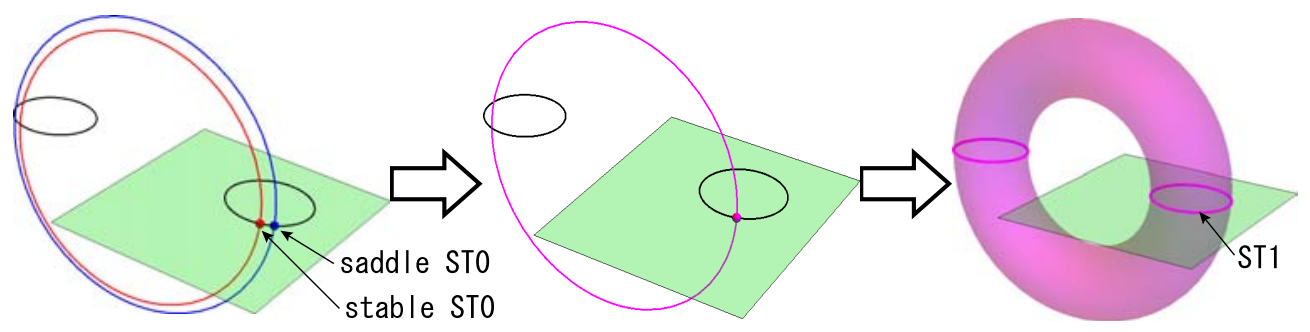

(a)

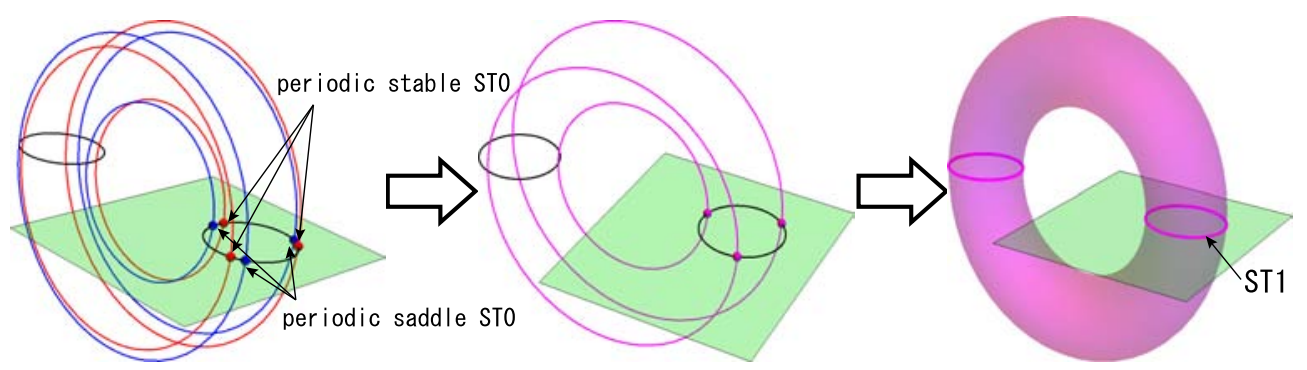

(b)

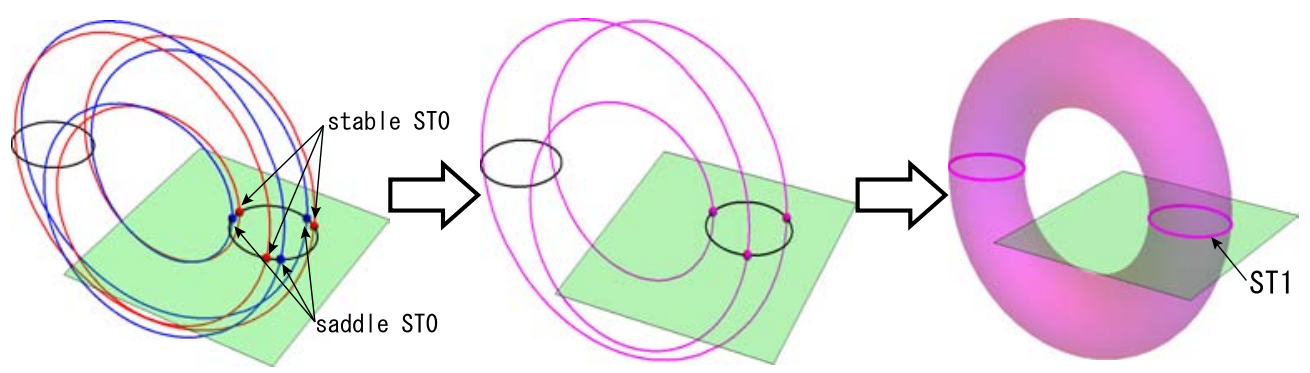

(c)

Fig. 11. Schematics of MTdSNC/FTdSNC bifurcations. (a) MTdSNC/FTdSNC for a simple MTd/FTd, (b) MTdSNC for a periodic MTd and (c) MTdSNC/FTdSNC for a winding MTd/FTd.

on a two-dimensional space have one-dimensional unstable sets $W^{u}$ s and one-dimensional stable sets $W^{s}$ s which form a circle by heteroclinic connections, which is called a heteroclinic circle. We assume that the product of the two eigenvalues of saddle is greater than 1 , and inside the heteroclinic circle, there exists a repelling MT0 with complex eigenvalues. When a parameter changes, $W^{u}$ s come close to and touch $W^{s}$ s from the outside, then intersect transversally with $W^{s} \mathrm{~s}$. Further, $W^{u} \mathrm{~s}$ touch $W^{s} \mathrm{~s}$ from the inside, then separate from $W^{s} \mathrm{~s}$. At a parameter value in an interval that $W^{u}$ s intersect transversally with $W^{s} \mathrm{~s}$, tangled MT1 is created, because parts of $W^{u}$ s inside $W^{s}$ s converge to MT1. The saddle fixed point remains through the bifurcation. ${ }^{12}$
We extend this concept to heteroclinic cycle bifurcations of MTd (abbr. MTdHCC) and FTd (abbr. FTdHCC); that is, saddle MTds/FTds have $(d+1)$-dimensional unstable sets $W^{u} \mathrm{~S}$ and $(N-1)$ dimensional stable sets $W^{s}$ s that form a $(d+$ 1)-dimensional torus by heteroclinic connections, which is called a heteroclinic torus. We assume that the sum of the positive LE and the maximum negative LE of saddle MTd/FTd is positive, and inside the heteroclinic torus, there exists a "repelling" MTd/FTd which has a positive LE $\lambda$ with $\mathrm{M}(\lambda)=2$, zero LE with $\mathrm{M}(0)=d$, and other LEs are negative. When a parameter changes, $W^{u}$ s come close to and touch $W^{s}$ s from the outside, then intersect transversally with $W^{s}$ s. Further, $W^{u}$ s touch $W^{s}$ s from the inside, then separate

\footnotetext{
${ }^{12}$ In the inverse of this bifurcation, MT1 touches the saddle MT0s and disappear, and the orbit on MT1 jumps to other attractors. This behavior is well known as phase locking of MT1 with hysteresis.
} 


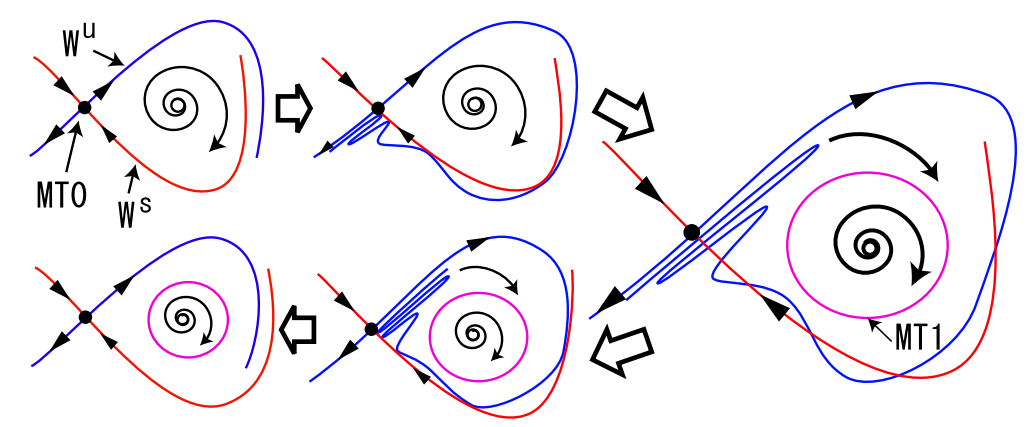

(a)

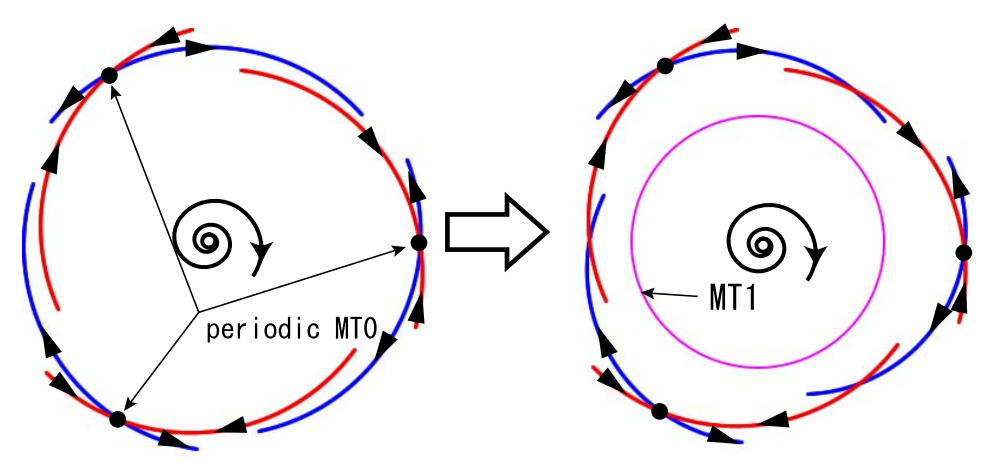

(b)

Fig. 12. Schematics of MTOHCC bifurcations. (a) MTOHCC for a simple MT0 and (b) MTOHCC for a periodic MT0.

from $W^{s}$ s. At a parameter value in an interval that $W^{u}$ s intersect transversally with $W^{s} \mathrm{~s}, \mathrm{MT}(d+$ $1) / \mathrm{FT}(d+1)$ is created, because parts of tangled $W^{u}$ s inside $W^{s}$ s converge to $\mathrm{MT}(d+1) / \mathrm{FT}(d+$ 1). The saddle MTds/FTds remain through the bifurcation.

Figure 13 illustrates $\mathrm{MT} d \mathrm{HCC} / \mathrm{FT} d \mathrm{HCC}$ in $\mathbb{R}^{N}$ where $N \geq d+2$. The black circles denote saddle MTds/FTds. The green rectangles denote codimension $d$ sections $\Sigma$. The white points denote ST0s on $\Sigma$. The red curves denote the stable sets of ST0s, which are intersections of the $(N-1)$ dimensional stable sets with $\Sigma$. The blue curves denote the unstable sets of ST0s, which are intersections of the $(d+1)$-dimensional unstable sets with $\Sigma$. The gray broken circle denotes the "repelling" $\mathrm{MTd} / \mathrm{FT} d$. The magenta tori and circles denote $\mathrm{MT}(d+1)$ and ST1, respectively. Figure 13(a) illustrates $\mathrm{MT} d \mathrm{HCC} / \mathrm{FT} d \mathrm{HCC}$ for a simple MTd/FTd. Figure 13(b) illustrates MTdHCC for a periodic $\mathrm{MTd}$. Figure 13(c) illustrates MTdHCC/FTdSNC for a winding $\mathrm{MT} d / \mathrm{FT} d$.

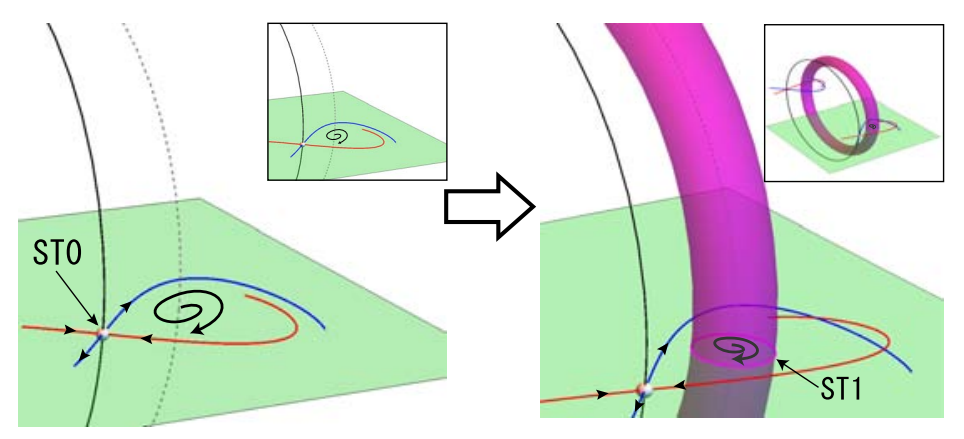

(a)

Fig. 13. Schematics of MTdHCC/FTdHCC bifurcations. (a) MTdHCC/FTdHCC for a simple MTd/FTd, (b) MTdHCC for a periodic MTd and (c) MTdHCC/FTdSNC for a winding MTd/FTd. 


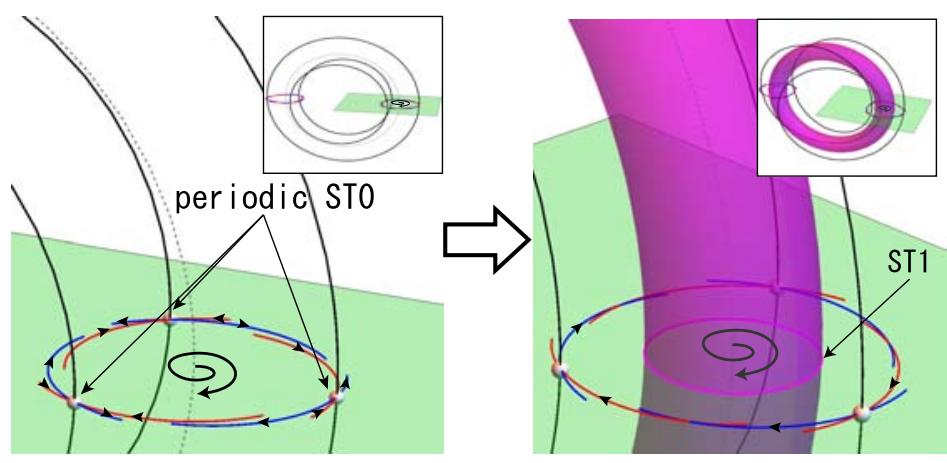

(b)

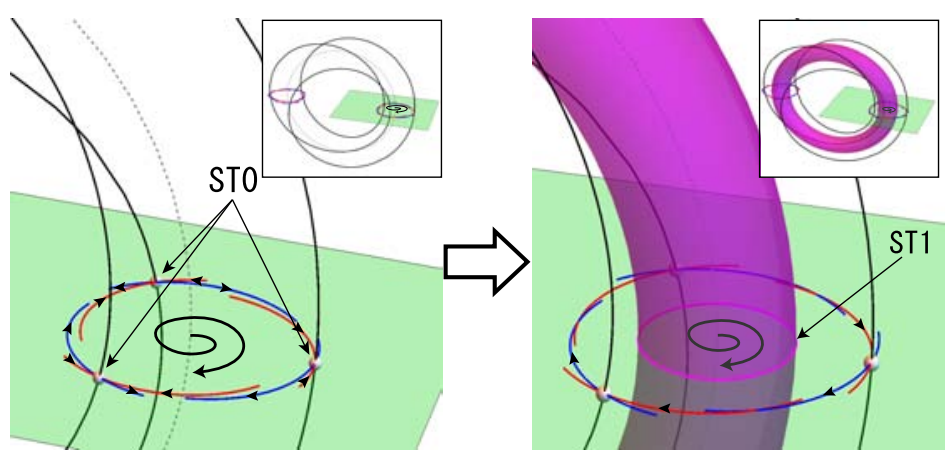

(c)

Fig. 13. (Continued)

For a numerical analysis of $\mathrm{MT} d \mathrm{HCC} / \mathrm{FT} d \mathrm{HCC}$, we observe bifurcations of ST0 that are intersections of a codimension $d$ section $\Sigma$ with MTd/FTd. On $\Sigma$, saddle ST0s have one-dimensional unstable sets and $(N-d-1)$-dimensional stable sets that form a circle by heteroclinic connections. When a parameter changes, the stable sets intersect transversally with the unstable sets, and ST1 is created on $\Sigma$. The saddle ST0s remain through the bifurcation. We call this phenomenon the heteroclinic cycle bifurcation of ST0 (abbr. STOHCC).

\section{Bifurcations in Discrete-Time Dynamical Systems}

In this section, we investigate examples of normal bifurcations of MT2 and MT3, and ones of resonance bifurcations of MT1 and MT2. In oneparameter and two-parameter Lyapunov diagrams, the $n$-fold multiplicity of both the DLE and zero LE is denoted by $\mathrm{M} n$. The notation $\mathrm{M}(0)$ is also used to denote the multiplicity of a zero LE. The slice radii of codimension one and codimension two sections are fixed as 0.001, unless otherwise specified. Also, we have used the condition $|\lambda|<0.001$ to determine a zero LE $\lambda$ for two-parameter Lyapunov diagrams, unless otherwise stated.

\subsection{Saddle-node bifurcation of MT2 (MT2SN)}

We present an example of MT2SN. Consider the following discrete-time dynamical system on $\mathbb{R}^{5}$ :

$$
\left\{\begin{aligned}
x_{0}(t+1)= & r_{1} \cos \theta_{1} x_{0}(t)-r_{1} \sin \theta_{1} x_{1}(t) \\
& -\left(r_{1}-0.9\right)\left(x_{0}^{2}(t)+x_{1}^{2}(t)\right)+e_{1} x_{2}(t), \\
x_{1}(t+1)= & r_{1} \sin \theta_{1} x_{0}(t)+r_{1} \cos \theta_{1} x_{1}(t) \\
& -\left(r_{1}-0.9\right)\left(x_{0}^{2}(t)+x_{1}^{2}(t)\right), \\
x_{2}(t+1)= & c \tanh x_{2}(t)+d+e_{2} x_{3}(t), \\
x_{3}(t+1)= & r_{2} \cos \theta_{2} x_{3}(t)-r_{2} \sin \theta_{2} x_{4}(t) \\
& -\left(r_{2}-0.9\right)\left(x_{3}^{2}(t)+x_{4}^{2}(t)\right), \\
x_{4}(t+1)= & r_{2} \sin \theta_{2} x_{3}(t)+r_{2} \cos \theta_{2} x_{4}(t) \\
& -\left(r_{2}-0.9\right)\left(x_{3}^{2}(t)+x_{4}^{2}(t)\right),
\end{aligned}\right.
$$

where $c$ and $d$ are the bifurcation parameters, and the other parameters are fixed as $r_{1}=1.10$, 


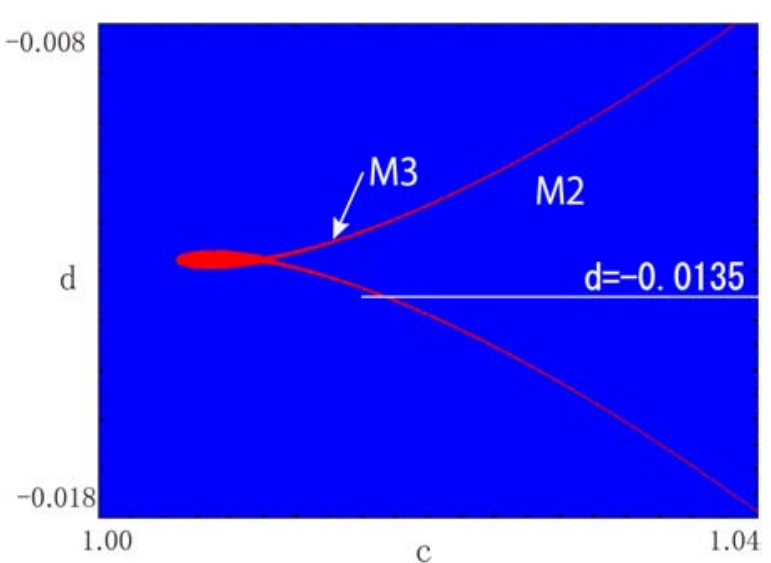

(a)

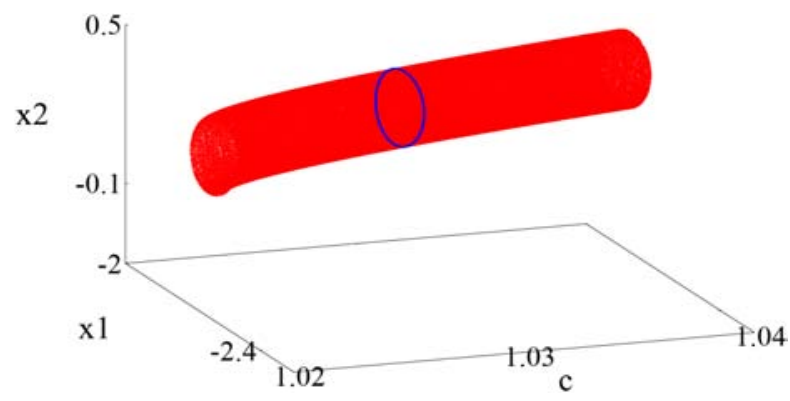

(c)

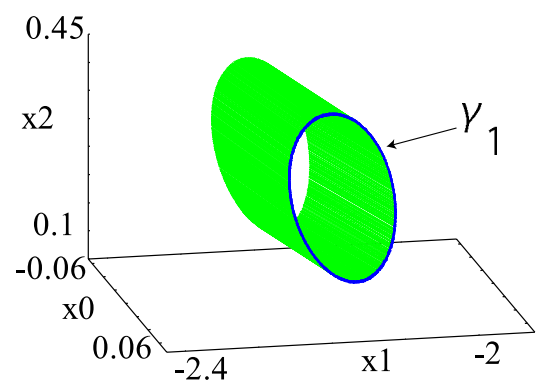

(e)

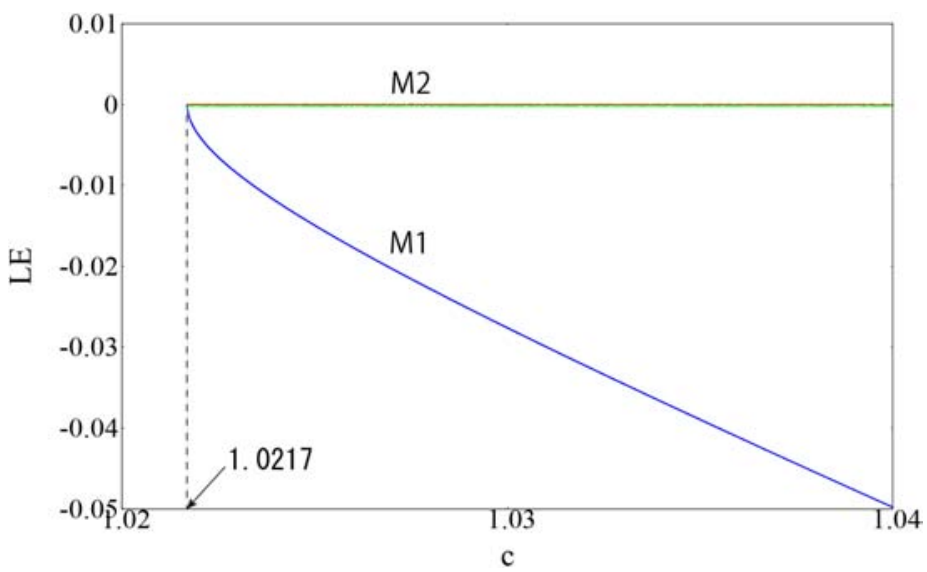

(b)

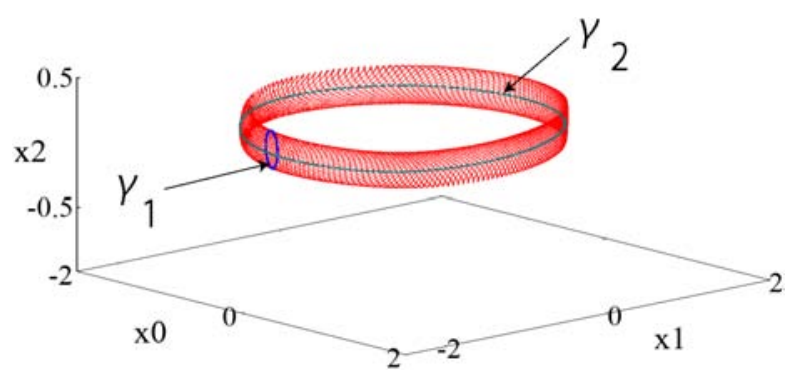

(d)

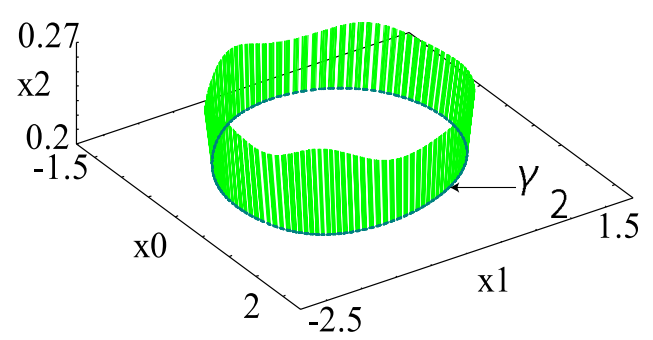

(f)

Fig. 14. MT2SN bifurcations. (a) Two-parameter Lyapunov diagram. The red and blue denote regions of $\mathrm{M}(0)=3$ and 2 , respectively. (b) One-parameter Lyapunov diagram at $d=-0.0135$. (c) One-parameter bifurcation diagram of ST1 on $\Sigma_{1}=\left\{x_{0}=0, x_{1}<0\right\}$ in $\left(c, x_{1}, x_{2}\right)$-space. (d) A stable MT2 in $\left(x_{0}, x_{1}, x_{2}\right)$-space at $c=1.03$. The blue circle $\gamma_{1}$ presents ST1 on $\Sigma_{1}=\left\{x_{0}=0, x_{1}<0\right\}$. The cyan circle $\gamma_{2}$ presents ST1 on $\Sigma_{2}=\left\{x_{4}=0, x_{3}>0\right\}$. (e) A type $A^{+}$DLB of $\gamma_{1}$ at $c=1.03$. (f) A type $A^{+}$DLB of $\gamma_{2}$ at $c=1.03$.

$\theta_{1}=70.1 \times \pi / 180, e_{1}=0.10, e_{2}=0.10, r_{2}=1.04$, and $\theta_{2}=58.7 \times \pi / 180$.

Figure 14(a) presents a two-parameter Lyapunov diagram of Eqs. (14) for $1.00 \leq c \leq 1.04$ and $-0.018 \leq d \leq-0.008$. The red and blue are used to denote regions of $\mathrm{M}(0)=3$ and 2 , respectively. The MT2SN bifurcation set is approximately denoted by the red region, because the multiplicity of the zero LE M(0) increases from two to three at the MT2SN bifurcation points where we have used the condition $|\lambda|<0.005$ to determine the zero LE $\lambda$. The white line in Fig. 14(a) represents the domain of the one-parameter Lyapunov diagram in Fig. 14(b) for $1.02 \leq c \leq 1.04$ at $d=$ -0.0135 . We observe that the DLE touches zero at $c=1.0217$. Figure 14(c) presents a one-parameter 
bifurcation diagram of ST1 on a codimension one section $\Sigma_{1}=\left\{x_{0}=0, x_{1}<0\right\}$ in $\left(c, x_{1}, x_{2}\right)$-space, for $1.02 \leq c \leq 1.04$ at $d=-0.0135$. A stable MT2 and a saddle MT2 collide at $c=1.0217$, and disappear when $c<1.0217$. That is, an MT2SN bifurcation occurs at $c=1.0217$. Figure $14(\mathrm{~d})$ presents a stable MT2 in $\left(x_{0}, x_{1}, x_{2}\right)$-space at $c=1.03$, prior to the MT2SN bifurcation. The blue circle $\gamma_{1}$ represents ST1 on $\Sigma_{1}=\left\{x_{0}=0, x_{1}<0\right\}$. The cyan circle $\gamma_{2}$ represents ST1 on $\Sigma_{2}=\left\{x_{4}=0, x_{3}>0\right\}$. Figures 14(e) and 14(f) present DLBs of $\gamma_{1}$ and $\gamma_{2}$, respectively, which are both of type $A^{+}$.

From these results, we observe an ST1SN bifurcation of $\gamma_{1}$ and $\gamma_{2}$. Hence, an MT2SN bifurcation occurs.

\subsection{Period doubling bifurcation of MT2 (MT2PD)}

Next, as an example of MT2PD, we analyze the same system as Eq. (14). In the following calculation, $c$ and $\theta_{1}$ are the bifurcation parameters, and other parameters are fixed as $r_{1}=1.10, d=0.0$, $e_{1}=0.10, e_{2}=0.25, r_{2}=1.04$, and $\theta_{2}=58.7 \times$ $\pi / 180$.

Figure 15(a) presents a two-parameter Lyapunov diagram of Eq. (14) for $-1.1 \leq c \leq-0.9$ and $70 \times \pi / 180 \leq \theta_{1} \leq 80 \times \pi / 180$. The red, blue, and yellow regions denote $\mathrm{M}(0)=3,2$, and 1 , respectively. The MT2PD bifurcation set is approximately denoted by the red region, because the multiplicity of the zero LE M(0) increases from two to three at the MT2PD bifurcation points. The white line represents the domain of the one-parameter Lyapunov diagram in Fig. 15(b) for $-1.1 \leq c \leq$ -0.9 at $\theta_{1}=74 \times \pi / 180$. We observe that the DLE touches zero at $c=-1.0185$. Figure $15(\mathrm{c})$ presents a one-parameter bifurcation diagram of ST1 on a codimension one section $\Sigma_{1}=\left\{x_{0}=0, x_{1}<0\right\}$ in $\left(c, x_{1}, x_{2}\right)$-space for $-1.1 \leq c \leq-0.9$ at $\theta_{1}=$ $74 \times \pi / 180$. An ST1 at $c=-0.95$ and a twoperiodic ST1 at $c=-1.05$ are denoted by blue circles. We observe an ST1PD bifurcation of the ST1. Figure 15(d) presents a one-parameter bifurcation diagram of another ST1 on a codimension one section $\Sigma_{2}=\left\{x_{4}=0, x_{3}>0\right\}$ in $\left(c, x_{0}, x_{1}+10 x_{2}\right)$ space, for $-1.1 \leq c \leq-0.9$ at $\theta_{1}=74 \times \pi / 180$. We also observe an ST1PD bifurcation of the ST1. Figure $15(\mathrm{e})$ presents a stable MT2 in $\left(x_{0}, x_{1}, x_{2}\right)$-space at $c=-0.95$, prior to the MT2PD bifurcation. The blue circle $\gamma_{1}$ and the green circle $\gamma_{2}$ represent ST1s on $\Sigma_{1}$ and $\Sigma_{2}$, respectively. Figures $15(\mathrm{~g})$ and $15(\mathrm{~h})$ present DLBs of $\gamma_{1}$ under the once-iterated map and the twice-iterated map, respectively, at $c=-0.95$. The former is an annulus with two sides, and the latter is an annulus with one side. Hence, the DLB of $\gamma_{1}$ is of type $A^{-}$. Similarly, Figs. 15(i) and 15(j) present DLBs of $\gamma_{2}$ under the once-iterated map and

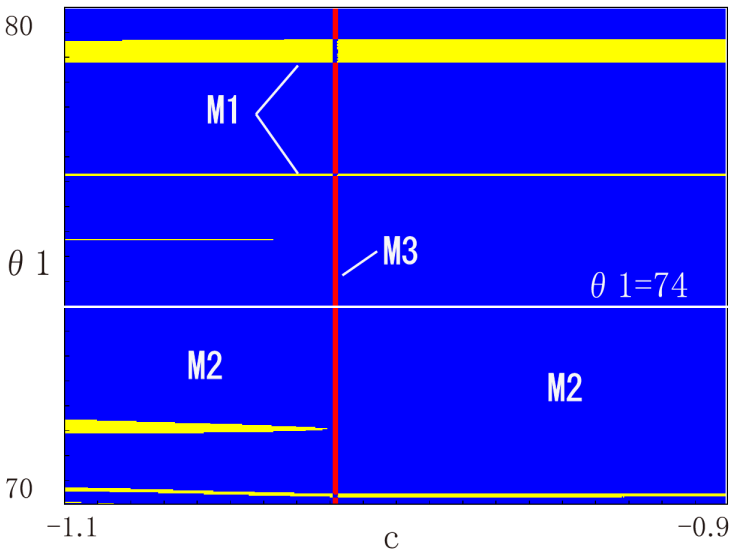

(a)

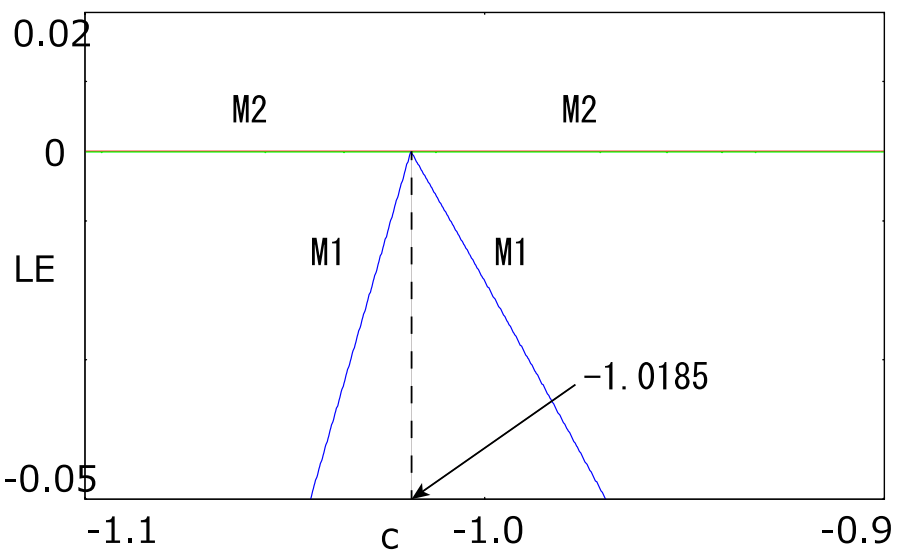

(b)

Fig. 15. MT2PD bifurcations. (a) Two-parameter Lyapunov diagram. The red, blue and yellow denote regions of $\mathrm{M}(0)=3$, 2, and 1, respectively. (b) One-parameter Lyapunov diagram for $-1.1 \leq c \leq-0.9$ at $\theta_{1}=74 \times \pi / 180$. (c) One-parameter bifurcation diagram of ST1 on $\Sigma_{1}=\left\{x_{0}=0, x_{1}<0\right\}$ in $\left(c, x_{1}, x_{2}\right)$-space. (d) One-parameter bifurcation diagram of ST1 on $\Sigma_{2}=\left\{x_{4}=0, x_{3}>0\right\}$ in $\left(c, x_{0}, x_{1}+10 x_{2}\right)$-space. (e) A stable MT2 in $\left(x_{0}, x_{1}, x_{2}\right)$-space at $c=-0.95$ before the MT2PD bifurcation. The blue circle $\gamma_{1}$ represents ST1 on $\Sigma_{1}$. The green circle $\gamma_{2}$ represents ST1 on $\Sigma_{2}$. (f) A two-periodic MT2 in $\left(x_{0}, x_{1}, x_{2}\right)$-space at $c=-1.05$ after the MT2PD bifurcation. The blue and green circles present two-periodic ST1s on $\Sigma_{1}$ and $\Sigma_{2}$, respectively. (g) and (h) DLBs of $\gamma_{1}$ under the once-iterated map and the twice-iterated map, respectively, at $c=-0.95$. (i) and (j) DLBs of $\gamma_{2}$ under the once-iterated map and the twice-iterated map, respectively, at $c=-0.95$. 


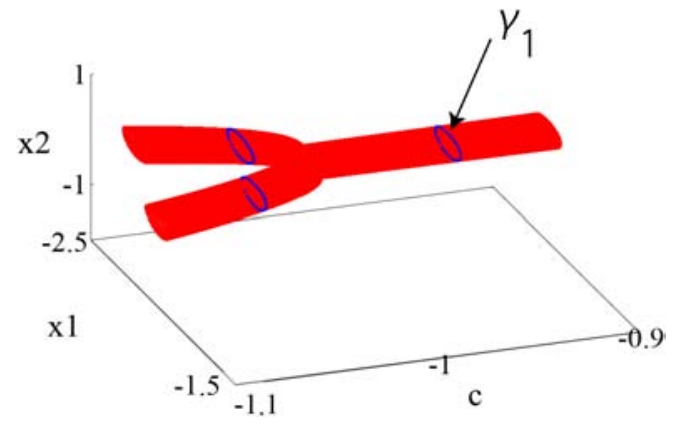

(c)

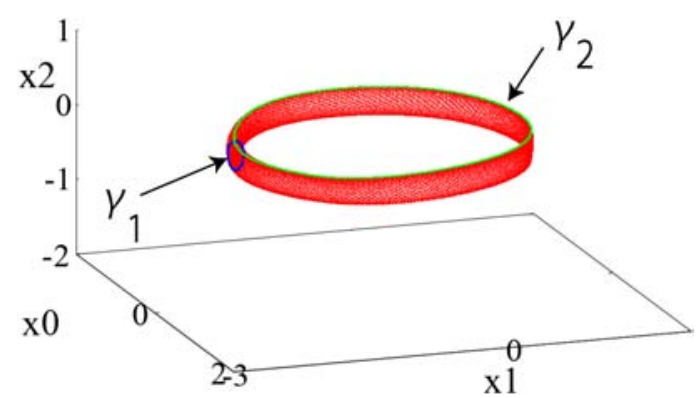

(e)

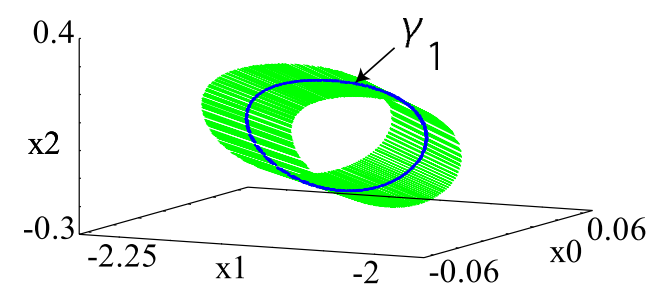

(g)

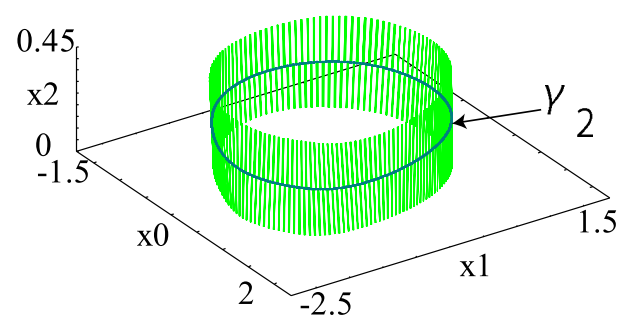

(i)

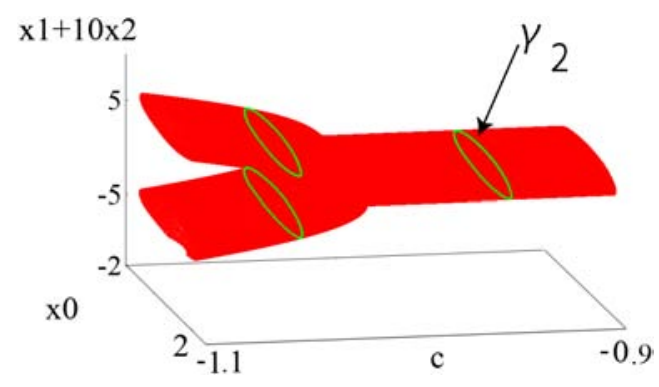

(d)

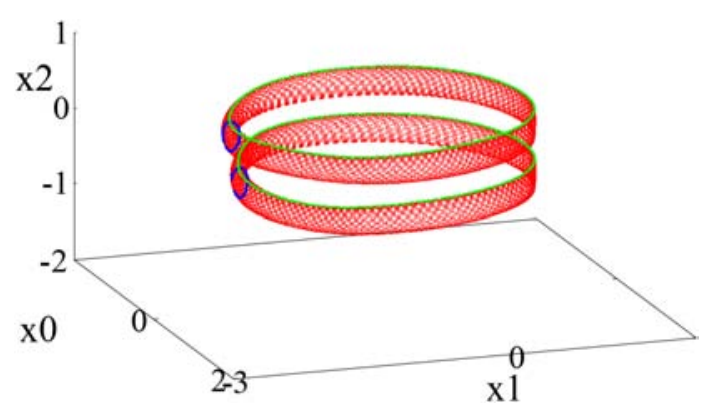

(f)

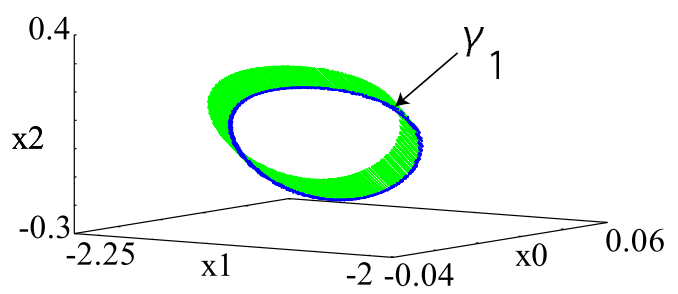

(h)

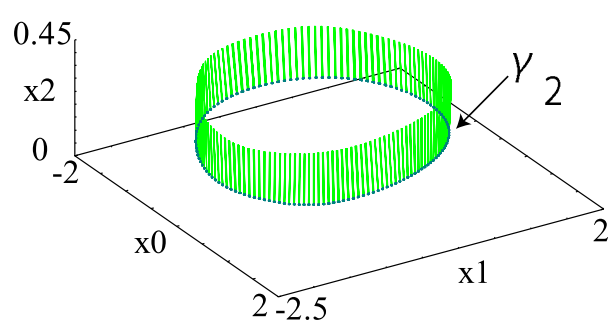

(j)

Fig. 15. (Continued) 
the twice-iterated map, respectively, at $c=-0.95$. The former is an annulus with two sides, and the latter is an annulus with one side. Hence, the DLB of $\gamma_{2}$ is also of type $A^{-}$. Figure $15(\mathrm{f})$ presents a twoperiodic MT2 in $\left(x_{0}, x_{1}, x_{2}\right)$-space at $c=-1.05$, after the MT2PD bifurcation. The blue and green circles represent two-periodic ST1s on $\Sigma_{1}$ and $\Sigma_{2}$, respectively.
From these results, we observe the ST1PD bifurcation of $\gamma_{1}$ and $\gamma_{2}$. Hence, the MT2PD bifurcation occurs.

\subsection{Double covering bifurcation of MT2 (MT2DC)}

Here, we present an example of MT2DC. Consider the following discrete-time dynamical system on $\mathbb{R}^{5}$ :

$$
\left\{\begin{array}{l}
x_{0}(t+1)=r_{1} \cos \theta_{1} x_{0}(t)-r_{1} \sin \theta_{1} x_{1}(t)-\left(r_{1}-0.9\right)\left(x_{0}^{2}(t)+x_{1}^{2}(t)\right)+e_{1} x_{2}(t)+e_{2} x_{3}(t), \\
x_{1}(t+1)=r_{1} \sin \theta_{1} x_{0}(t)+r_{1} \cos \theta_{1} x_{1}(t)-\left(r_{1}-0.9\right)\left(x_{0}^{2}(t)+x_{1}^{2}(t)\right) \\
x_{2}(t+1)=x_{0}(t) \\
x_{3}(t+1)=r_{2} \cos \theta_{2} x_{3}(t)-r_{2} \sin \theta_{2} x_{4}(t)-\left(r_{2}-0.9\right)\left(x_{3}^{2}(t)+x_{4}^{2}(t)\right), \\
x_{4}(t+1)=r_{2} \sin \theta_{2} x_{3}(t)+r_{2} \cos \theta_{2} x_{4}(t)-\left(r_{2}-0.9\right)\left(x_{3}^{2}(t)+x_{4}^{2}(t)\right)
\end{array}\right.
$$

where $r_{1}$ and $\theta_{1}$ are the bifurcation parameters, and the other parameters are fixed as $r_{2}=1.02$, $\theta_{2}=70.1 \times \pi / 180, e_{1}=-0.20$, and $e_{2}=0.01$.

Figure 16(a) presents a two-parameter Lyapunov diagram of Eqs. (15) for $1.090 \leq r_{1} \leq 1.096$ and $56.0 \times \pi / 180 \leq \theta_{1} \leq 60.0 \times \pi / 180$. The red, blue, and yellow regions denote $\mathrm{M}(0)=3,2$, and 1 , respectively. The MT2DC bifurcation set is approximately denoted by the red region, because the multiplicity of zero $\mathrm{LE} \mathrm{M}(0)$ increases from two to three at the MT2DC bifurcation points. The white line represents the domain of the one-parameter Lyapunov diagram in Fig. 16(b) for $1.090 \leq r_{1} \leq$ 1.096 at $\theta_{1}=58.0 \times \pi / 180$. We observe that the DLE touches zero at $r_{1}=1.093314$. Figure 16(c) presents a one-parameter bifurcation diagram of ST1 on a codimension one section $\Sigma_{1}=\left\{x_{0}=\right.$ $\left.0, x_{1}<0\right\}$ in $\left(r_{1}, x_{2}, x_{3}\right)$-space for $1.090 \leq r_{1} \leq$ 1.096 at $\theta_{1}=58.0 \times \pi / 180$. An ST1 at $r_{1}=1.091$

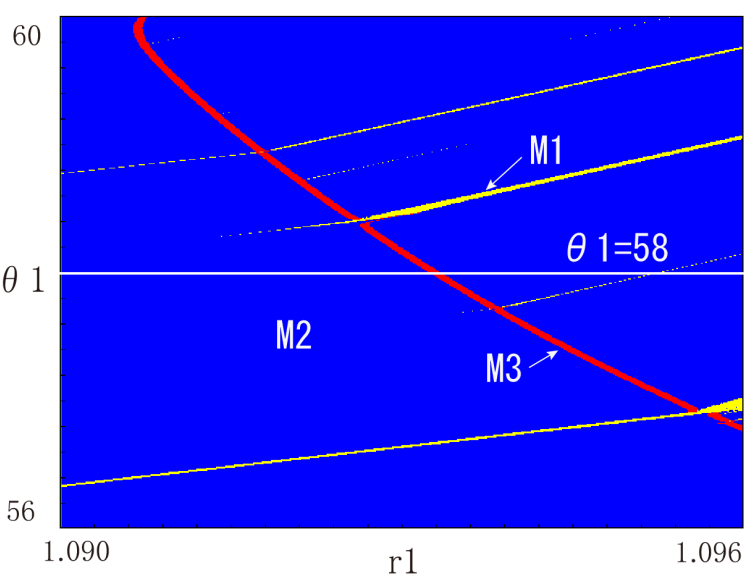

(a)

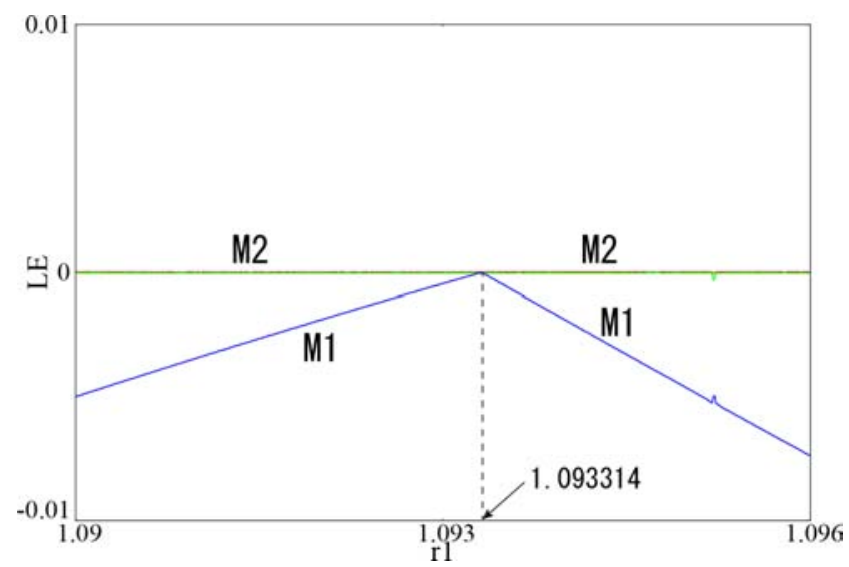

(b)

Fig. 16. MT2DC bifurcations. (a) Two-parameter Lyapunov diagram. The red, blue, and yellow denote regions of $\mathrm{M}(0)=3$, 2, and 1, respectively. (b) One-parameter Lyapunov diagram for $1.090 \leq r_{1} \leq 1.096$ at $\theta_{1}=58.0 \times \pi / 180$. (c) One-parameter bifurcation diagram of ST1 on $\Sigma_{1}=\left\{x_{0}=0, x_{1}<0\right\}$ in $\left(r_{1}, x_{2}, x_{3}\right)$-space. (d) One-parameter bifurcation diagram of ST1 on $\Sigma_{2}=\left\{x_{3}=0, x_{4}>0\right\}$ in $\left(r_{1}, x_{0}, x_{1}\right)$-space. (e) A stable MT2 in $\left(x_{0}, x_{1}, x_{3}\right)$-space at $r_{1}=1.091$, prior to the MT2DC bifurcation. The blue circle $\gamma_{1}$ represents ST1 on $\Sigma_{1}$. The green circle $\gamma_{2}$ represents ST1 on $\Sigma_{2}$. (f) A double covering MT2 in $\left(x_{0}, x_{1}, x_{3}\right)$-space at $r_{1}=1.095$ after the MT2DC bifurcation. The blue circles represent double component ST1s on $\Sigma_{1}$, and the green circle presents a double covering ST1 on $\Sigma_{2} \cdot(\mathrm{g})$ and (h) DLBs of $\gamma_{1}$ under the once-iterated map and the twice-iterated map, respectively, at $r_{1}=1.091$. (i) and (j) DLBs of $\gamma_{2}$ under the once-iterated map and the twice-iterated map, respectively, at $r_{1}=1.091$. 


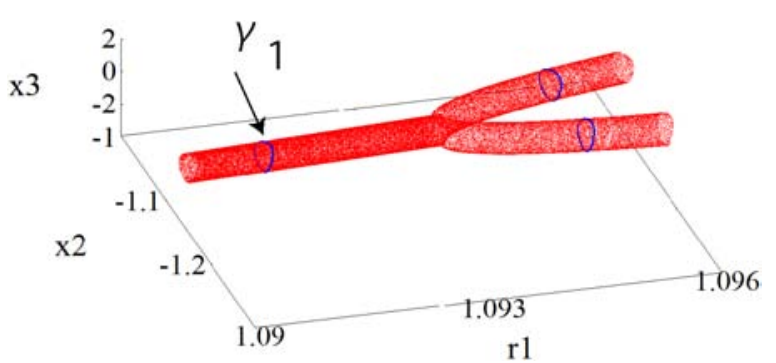

(c)

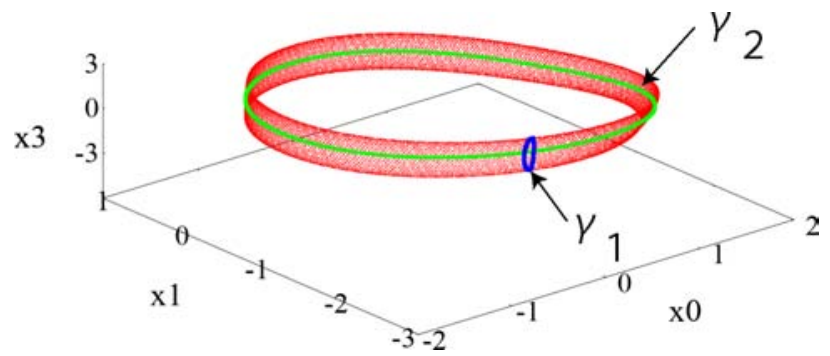

(e)

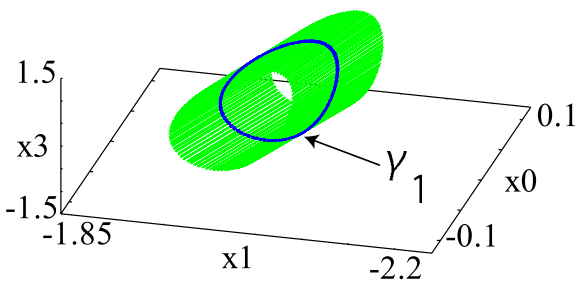

(g)

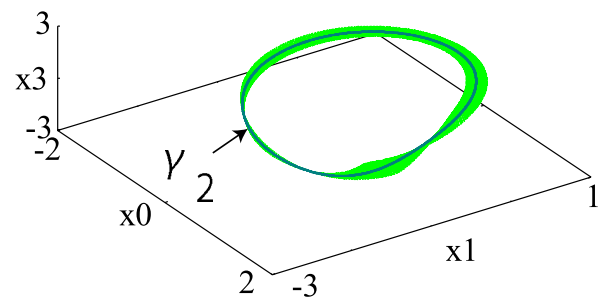

(i)

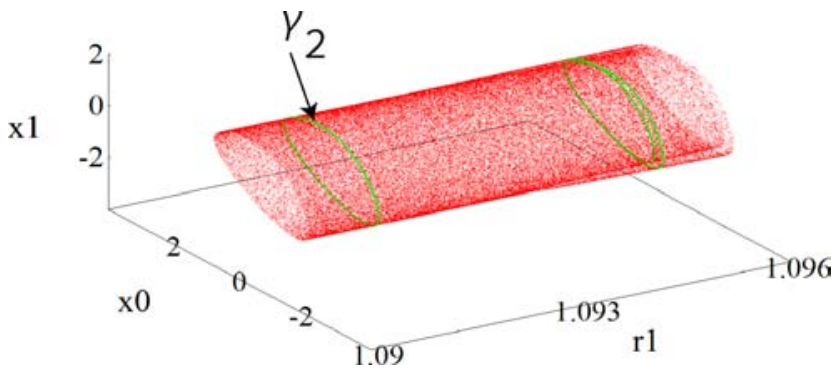

(d)

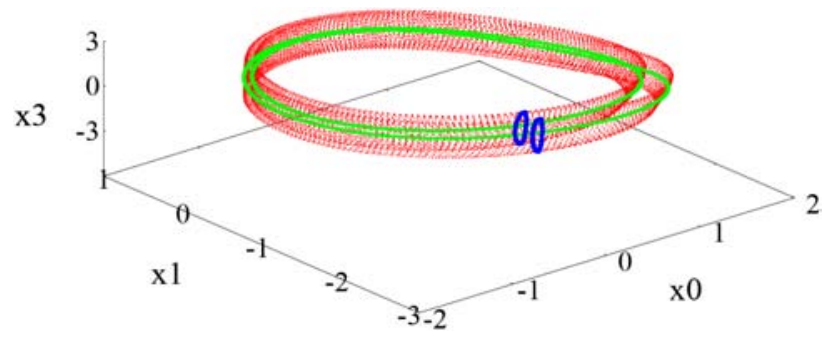

(f)

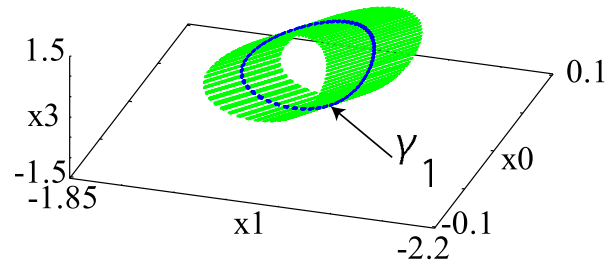

(h)

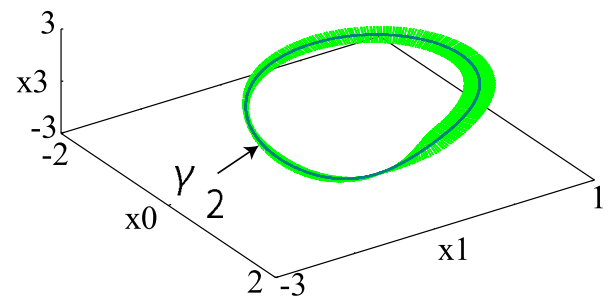

(j)

Fig. 16. (Continued)

and double component ST1s at $r_{1}=1.095$ are denoted by the blue circles. We observe an ST1CD bifurcation of the ST1. Figure 16(d) presents a oneparameter bifurcation diagram of another ST1 on a codimension one section $\Sigma_{2}=\left\{x_{3}=0, x_{4}>0\right\}$ in $\left(r_{1}, x_{0}, x_{1}\right)$-space for $1.090 \leq r_{1} \leq 1.096$ at $\theta_{1}=58.0 \times \pi / 180$. An ST1 at $r_{1}=1.091$ and a double covering ST1 at $r_{1}=1.095$ are denoted by the green circles. We observe an ST1DC bifurcation of the ST1. Figure 16(e) presents a stable MT2 in $\left(x_{0}, x_{1}, x_{3}\right)$-space at $r_{1}=1.091$, prior to an MT2DC bifurcation. The blue circle $\gamma_{1}$ and the green circle $\gamma_{2}$ represent ST1s on $\Sigma_{1}$ and $\Sigma_{2}$, respectively. Figures 16(g) and 16(h) present DLBs of $\gamma_{1}$ under the once-iterated map and the twice-iterated map, respectively, at $r_{1}=1.091$. Both the former and the latter are annuli with two sides. Hence, the DLB of $\gamma_{1}$ is of type $A^{*}$. Similarly, Figs. 16(i) and 16(j) present DLBs of $\gamma_{2}$ under the once-iterated map and the twice-iterated map, respectively, at $r_{1}=1.091$. 
Both the former and the latter are Möbius bands. Hence, the DLB of $\gamma_{2}$ is of type $M$. Figure 16(f) presents a double covering MT2 in $\left(x_{0}, x_{1}, x_{3}\right)$-space at $r_{1}=1.095$, after the MT2DC bifurcation. The blue circles represent double component ST1s on $\Sigma_{1}$, and the green circle represents a double covering ST1 on $\Sigma_{2}$.

From these results, we observe the ST1CD bifurcation of $\gamma_{1}$ and the ST1DC bifurcation of $\gamma_{2}$. Hence, the MT2DC bifurcation occurs.

\subsection{Neimark-Sacker bifurcation of MT2 (MT2NS)}

We present an example of MT2NS. Consider the following discrete-time dynamical system on $\mathbb{R}^{6}$ :

$$
\left\{\begin{aligned}
x_{0}(t+1)= & r_{1} \cos \theta_{1} x_{0}(t)-r_{1} \sin \theta_{1} x_{1}(t) \\
& -\left(r_{1}-0.9\right)\left(x_{0}^{2}(t)+x_{1}^{2}(t)\right)+e_{1} x_{2}(t), \\
x_{1}(t+1)= & r_{1} \sin \theta_{1} x_{0}(t)+r_{1} \cos \theta_{1} x_{1}(t) \\
& -\left(r_{1}-0.9\right)\left(x_{0}^{2}(t)+x_{1}^{2}(t)\right), \\
x_{2}(t+1)= & r_{2} \cos \theta_{2} x_{2}(t)-r_{2} \sin \theta_{2} x_{3}(t) \\
& -\left(r_{2}-0.9\right)\left(x_{2}^{2}(t)+x_{3}^{2}(t)\right) \\
& +e_{2} x_{0}(t)+e_{3} x_{4}(t), \\
x_{3}(t+1)= & r_{2} \sin \theta_{2} x_{2}(t)+r_{2} \cos \theta_{2} x_{3}(t) \\
& -\left(r_{2}-0.9\right)\left(x_{2}^{2}(t)+x_{3}^{2}(t)\right), \\
x_{4}(t+1)= & r_{3} \cos \theta_{3} x_{4}(t)-r_{3} \sin \theta_{3} x_{5}(t) \\
& -\left(r_{3}-0.9\right)\left(x_{4}^{2}(t)+x_{5}^{2}(t)\right), \\
x_{5}(t+1)= & r_{3} \sin \theta_{3} x_{4}(t)+r_{3} \cos \theta_{3} x_{5}(t) \\
& -\left(r_{3}-0.9\right)\left(x_{4}^{2}(t)+x_{5}^{2}(t)\right),
\end{aligned}\right.
$$

where $r_{2}$ and $\theta_{2}$ are bifurcation parameters, and the other parameters are fixed as $r_{1}=1.014583$, $\theta_{1}=59.281250 \times \pi / 180, e_{1}=0.02, e_{2}=0.02$, $e_{3}=0.10, r_{3}=1.04$, and $\theta_{3}=58.7 \times \pi / 180$.

Figure 17(a) presents a two-parameter Lyapunov diagram for $0.9 \leq r_{2} \leq 1.1$ and $56 \times \pi / 180 \leq$ $\theta_{2} \leq 80 \times \pi / 180$. The green, red and blue regions denote $\mathrm{M}(0)=4,3$ and 2, respectively. The MT2NS bifurcation set is approximately denoted by the green line, because the multiplicity of zero LE M(0) increases from two to four at the MT2NS bifurcation points. The white line represents the domain of the one-parameter Lyapunov diagram in Fig. 17(b) for $0.9 \leq r_{2} \leq 1.1$ at $\theta_{2}=72.1 \times \pi / 180$. We observe that the DLE touches zero at $r_{2}=1.0015$. Figure 17(c) presents a one-parameter bifurcation diagram of ST1 on a codimension one section $\Sigma_{1}=$ $\left\{x_{0}=0, x_{1}<0\right\}$ in $\left(r_{2}, x_{1}, x_{4}\right)$-space for $0.9 \leq$ $r_{2} \leq 1.1$ at $\theta_{2}=72.1 \times \pi / 180$. ST1 at $r_{2}=0.99$ and ST2 at $r_{2}=1.05$ are denoted by the blue circle and the blue torus. ST0 at $r_{2}=0.99$ and ST1 at $r_{2}=1.05$ that are on a codimension two section $\Sigma_{2}=\left\{x_{0}=0, x_{1}<0, x_{2}=0, x_{3}>0\right\}$ are denoted by the magenta point and the magenta circle. We observe an ST1NS bifurcation and an ST0NS bifurcation. Figure 17(d) presents a stable MT2 in $\left(x_{0}, x_{1}, x_{4}\right)$-space at $r_{2}=0.99$, prior to the MT2NS bifurcation. The blue circle $\gamma$ represents ST1 on $\Sigma_{1}$, and the magenta point $p$ represents ST0 on $\Sigma_{2}$. Figure $17(\mathrm{f})$ presents a DLB of $\gamma$ in $\left(x_{0}, x_{1}+0.1 x_{3}, x_{4}\right)$-space at $r_{2}=0.99$, prior to the MT2NS bifurcation. This is of type $F$. Figure 17(e) presents a stable MT3 in $\left(x_{0}, x_{1}, x_{4}\right)$-space at $r_{2}=1.05$ after the MT2NS bifurcation. The blue torus represents ST2 on $\Sigma_{1}$, and the magenta circle represents ST1 on $\Sigma_{2}$.

From these results, we observe the ST1NS bifurcation and the ST0NS bifurcation. Hence, the MT2NS bifurcation occurs.

\subsection{Saddle-node bifurcation of MT3 (MT3SN)}

We present an example of MT3NS. Consider the following discrete-time dynamical system on $\mathbb{R}^{7}$ :

$$
\left\{\begin{aligned}
x_{0}(t+1)= & r_{1} \cos \theta_{1} x_{0}(t)-r_{1} \sin \theta_{1} x_{1}(t) \\
& -\left(r_{1}-0.9\right)\left(x_{0}^{2}(t)+x_{1}^{2}(t)\right)+e_{1} x_{2}(t), \\
x_{1}(t+1)= & r_{1} \sin \theta_{1} x_{0}(t)+r_{1} \cos \theta_{1} x_{1}(t) \\
& -\left(r_{1}-0.9\right)\left(x_{0}^{2}(t)+x_{1}^{2}(t)\right), \\
x_{2}(t+1)= & c \tanh x_{2}(t)+d+e_{2} x_{3}(t), \\
x_{3}(t+1)= & r_{2} \cos \theta_{2} x_{3}(t)-r_{2} \sin \theta_{2} x_{4}(t) \\
& -\left(r_{2}-0.9\right)\left(x_{3}^{2}(t)+x_{4}^{2}(t)\right)+e_{3} x_{5}(t), \\
x_{4}(t+1)= & r_{2} \sin \theta_{2} x_{3}(t)+r_{2} \cos \theta_{2} x_{4}(t) \\
& -\left(r_{2}-0.9\right)\left(x_{3}^{2}(t)+x_{4}^{2}(t)\right), \\
x_{5}(t+1)= & r_{3} \cos \theta_{3} x_{5}(t)-r_{3} \sin \theta_{3} x_{6}(t) \\
& -\left(r_{3}-0.9\right)\left(x_{5}^{2}(t)+x_{6}^{2}(t)\right), \\
x_{6}(t+1)= & r_{3} \sin \theta_{3} x_{5}(t)+r_{3} \cos \theta_{3} x_{6}(t) \\
& -\left(r_{3}-0.9\right)\left(x_{5}^{2}(t)+x_{6}^{2}(t)\right),
\end{aligned}\right.
$$




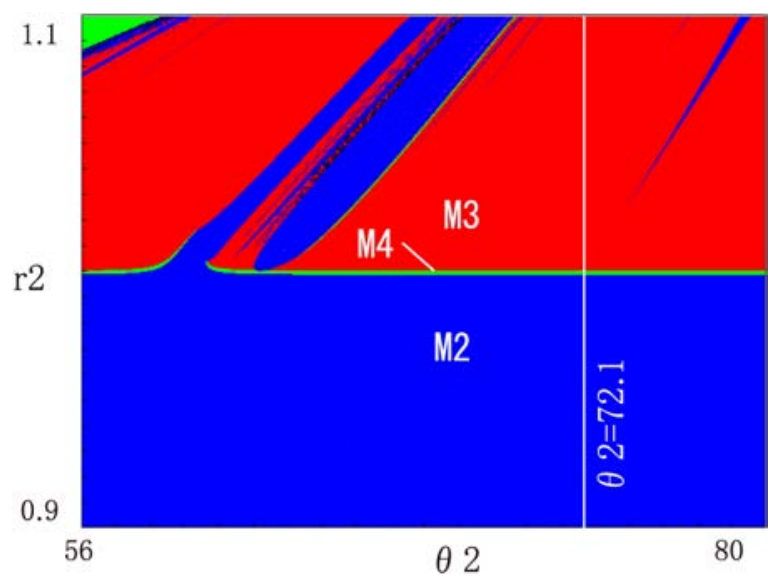

(a)

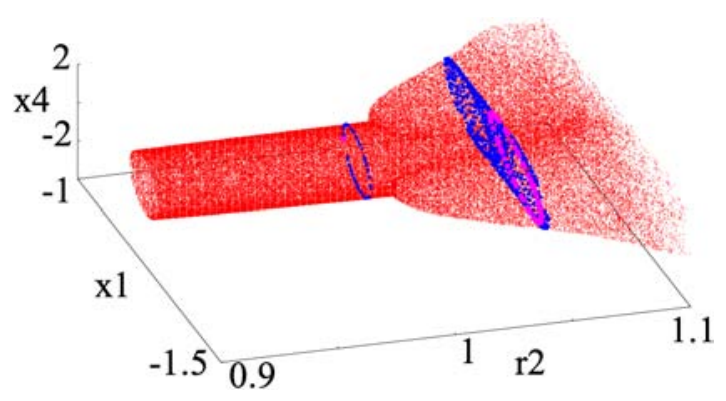

(c)

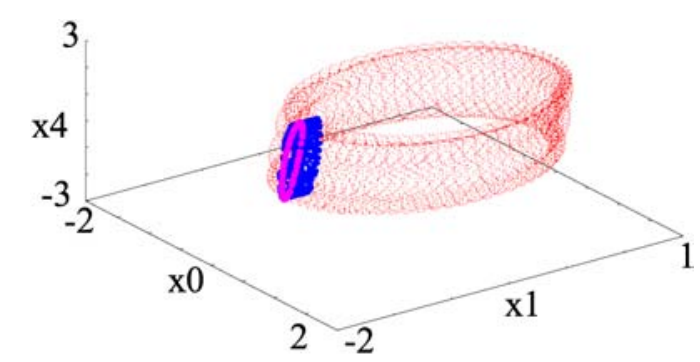

(e)

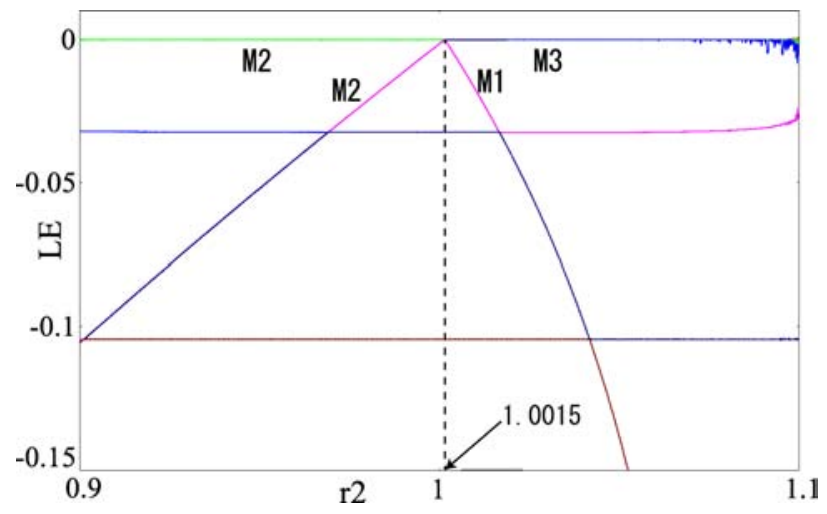

(b)

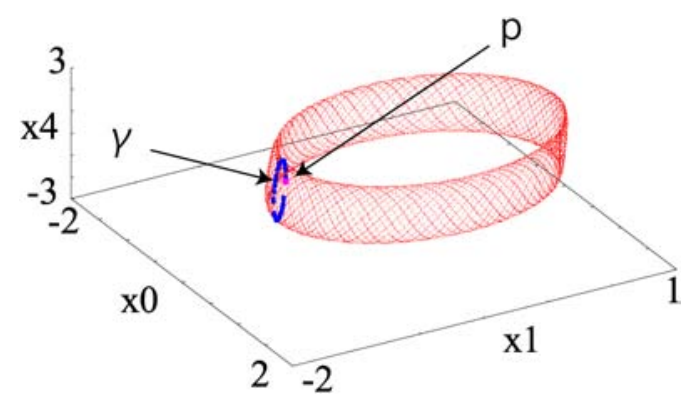

(d)

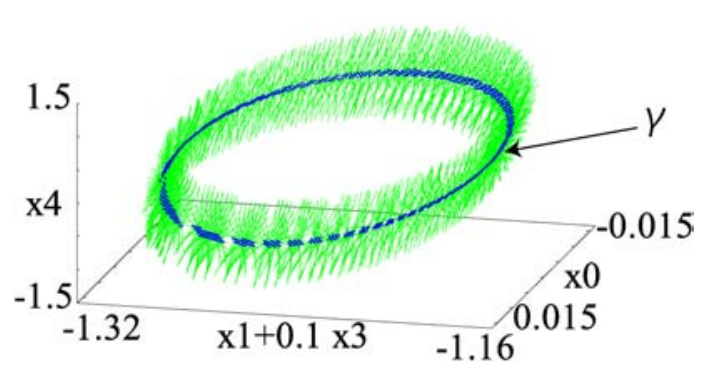

(f)

Fig. 17. MT2NS bifurcations. (a) Two-parameter Lyapunov diagram for $0.9 \leq r_{2} \leq 1.1$ and $56 \times \pi / 180 \leq \theta_{2} \leq 80 \times \pi / 180$. The green, red, and blue denote regions of $\mathrm{M}(0)=4,3$ and 2, respectively. (b) One-parameter Lyapunov diagram for $0.9 \leq r_{2} \leq 1.1$ at $\theta_{2}=72.1 \times \pi / 180$. (c) One-parameter bifurcation diagram of ST1 on $\Sigma_{1}=\left\{x_{0}=0, x_{1}<0\right\}$ in $\left(r_{2}, x_{1}, x_{4}\right)$-space. ST1 at $r_{2}=0.99$ and ST2 at $r_{2}=1.05$ are denoted by the blue circle and the blue torus. ST0 at $r_{2}=0.99$ and ST1 at $r_{2}=1.05$ that are on a codimension two section $\Sigma_{2}=\left\{x_{0}=0, x_{1}<0, x_{2}=0, x_{3}>0\right\}$ are denoted by the magenta point and the magenta circle. (d) A stable MT2 in $\left(x_{0}, x_{1}, x_{4}\right)$-space at $r_{2}=0.99$ before the MT2NS bifurcation. The blue circle $\gamma$ represents ST1 on $\Sigma_{1}$, and the magenta point $p$ presents ST0 on $\Sigma_{2}$. (e) A stable MT3 in $\left(x_{0}, x_{1}, x_{4}\right)$-space at $r_{2}=1.05$ after the MT2NS bifurcation. The blue torus represents ST2 on $\Sigma_{1}$, and the magenta circle represents ST1 on $\Sigma_{2}$. (f) A type $F$ DLB of $\gamma$ at $r_{2}=0.99$.

where $c$ and $d$ are the bifurcation parameters, and the other parameters are fixed as $r_{1}=1.10, \theta_{1}=$ $70.1 \times \pi / 180, e_{1}=0.10, e_{2}=0.10, e_{3}=0.01$ $r_{2}=1.04, \theta_{2}=58.7 \times \pi / 180, r_{3}=1.04$, and $\theta_{3}=56.7 \times \pi / 180$.

Figure 18(a) presents a two-parameter Lyapunov diagram of Eqs. (17) for $1.00 \leq c \leq 1.05$ and $-0.018 \leq d \leq-0.008$. The green and red regions denote $\mathrm{M}(0)=4$ and 3 , respectively. The MT3SN bifurcation set is approximately denoted by the green region, because the multiplicity of zero $\mathrm{LE} \mathrm{M}(0)$ increases from three to four at the MT3SN bifurcation points where we have used the condition $|\lambda|<0.005$ to determine the zero $\operatorname{LE} \lambda$. The white 


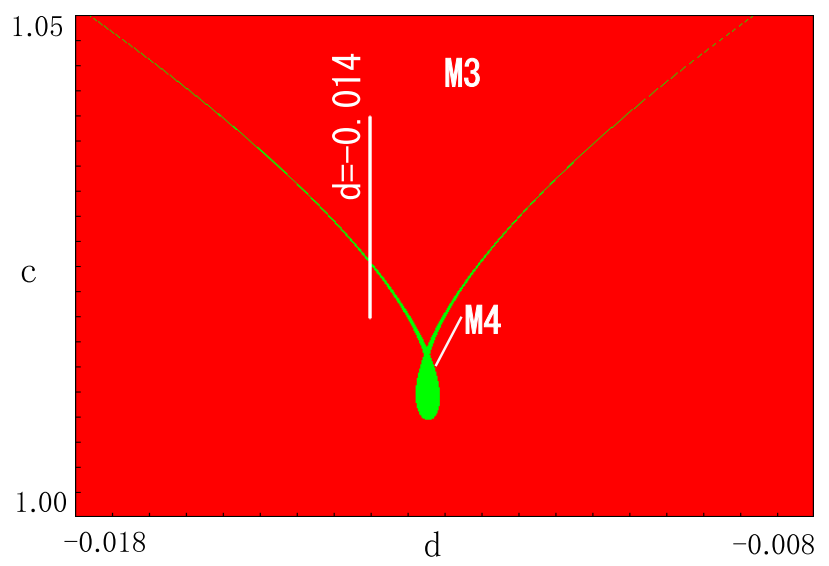

(a)

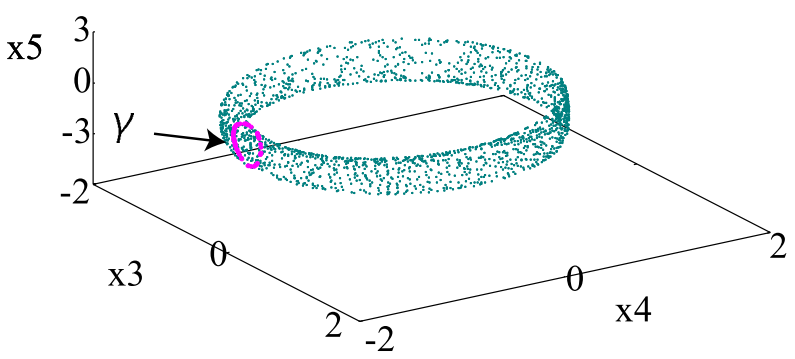

(c)

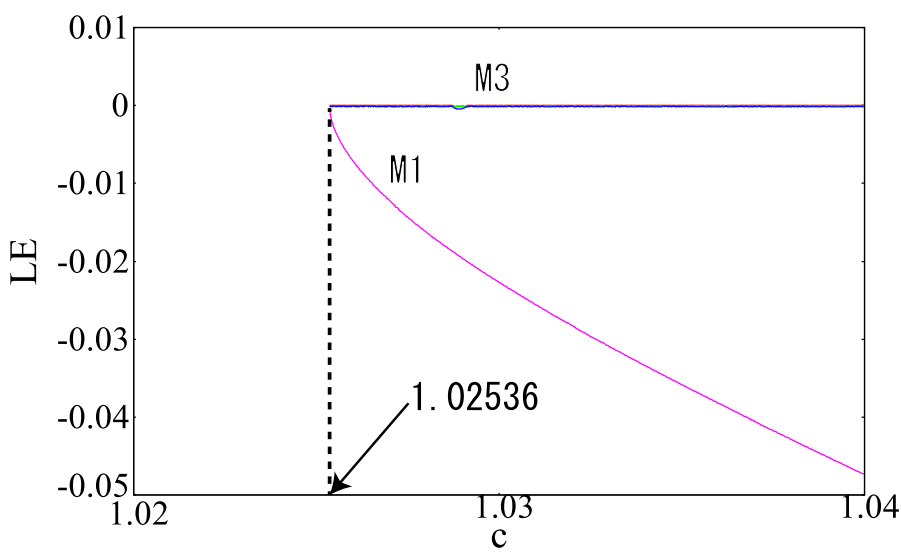

(b)

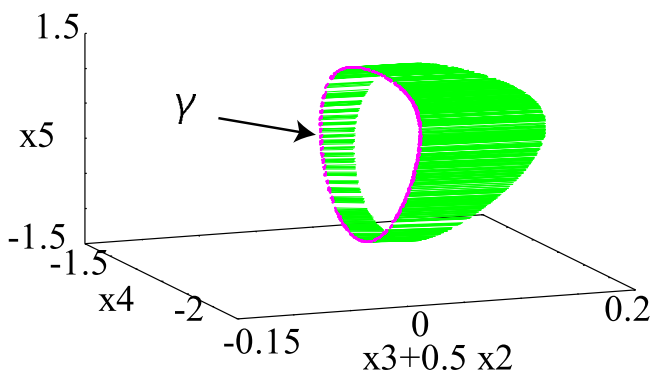

(d)

Fig. 18. MT3SN bifurcations. (a) Two-parameter Lyapunov diagram. The green and red denote regions of $\mathrm{M}(0)=4$ and 3 , respectively. (b) One-parameter Lyapunov diagram at $d=-0.014$. (c) ST2 on $\Sigma_{1}=\left\{x_{0}=0, x_{1}<0\right\}$ in $\left(x_{3}, x_{4}, x_{5}\right)$-space at $c=1.03$. The magenta circle $\gamma$ represents an ST1 on $\Sigma_{2}=\left\{x_{0}=0, x_{1}<0, x_{3}=0, x_{4}<0\right\}$. (d) A type $A^{+}$DLB of $\gamma$ in $\left(x_{3}+0.5 x_{2}, x_{4}, x_{5}\right)$-space at $c=1.03$.

line represents the domain of the one-parameter Lyapunov diagram in Fig. 18(b) for $1.02 \leq c \leq 1.04$ at $d=-0.014$. We observe that the DLE touches zero at $c=1.02536$. Figure 18(c) presents ST2 on a codimension one section $\Sigma_{1}=\left\{x_{0}=0, x_{1}<0\right\}$ in $\left(x_{3}, x_{4}, x_{5}\right)$-space at $c=1.03$. The magenta circle $\gamma$ represents ST1 on a codimension two section $\Sigma_{2}=\left\{x_{0}=0, x_{1}<0, x_{3}=0, x_{4}<0\right\}$. Figure 18(d) presents the DLB of $\gamma$ in $\left(x_{3}+0.5 x_{2}, x_{4}, x_{5}\right)$-space at $c=1.03$, which is of type $A^{+}$.

From these results, we observe the ST1SN bifurcation. Hence, the MT3SN bifurcation occurs at $c=1.02536$. That is, a stable MT3 and a saddle MT3 collide at $c=1.02536$, and vanish when $c<1.02536$.

\subsection{Period doubling bifurcation of MT3 (MT3PD)}

Next, as an example of MT3PD, we analyze the same system as Eqs. (17). In the following calculation, $c$ and $\theta_{1}$ are the bifurcation parameters, and the other parameters are fixed as $r_{1}=1.10$, $d=0.0, e_{1}=0.10, e_{2}=0.25, e_{3}=0.25, r_{2}=1.04$, $\theta_{2}=58.7 \times \pi / 180, r_{3}=1.04$, and $\theta_{3}=56.7 \times \pi / 180$.

Figure 19(a) presents a two-parameter Lyapunov diagram of Eqs. (17) for $-1.10 \leq c \leq-0.90$ and $70 \times \pi / 180 \leq \theta_{1} \leq 80 \times \pi / 180$. The green, red and blue regions denote $\mathrm{M}(0)=4,3$ and 2 , respectively. The MT3PD bifurcation set is approximately denoted by the green region, because the multiplicity of the zero $\mathrm{LE} \mathrm{M}(0)$ increases from three to four at the MT3PD bifurcation points. The white line represents the domain of the one-parameter Lyapunov diagram in Fig. 19(b) for $-1.05 \leq c \leq$ -0.95 at $\theta_{1}=74 \times \pi / 180$. We observe that the DLE touches zero at $c=-1.018750$. Figure 19(c) presents an ST2 on a codimension one section $\Sigma_{1}=\left\{x_{0}=0, x_{1}<0\right\}$ in $\left(x_{3}, x_{4}+x_{2}, x_{5}\right)$-space at $c=-1.0$, prior to the MT3PD bifurcation. The magenta circle $\gamma_{1}$ represents ST1 on a codimension two section $\Sigma_{2}=\left\{x_{0}=0, x_{1}<0, x_{3}=0, x_{4}<0\right\}$. The red circle $\gamma_{2}$ represents ST1 on a codimension 


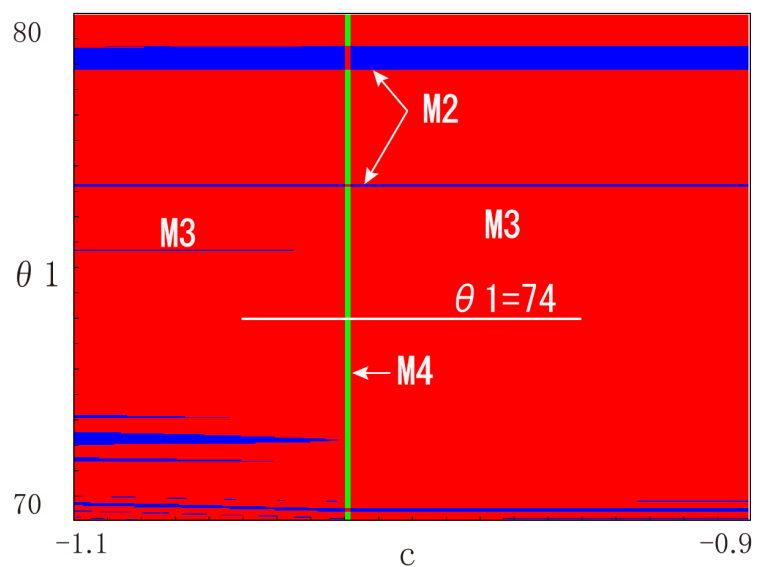

(a)

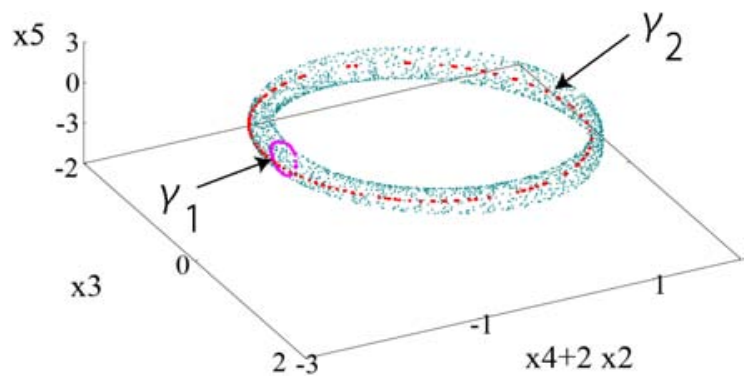

(c)

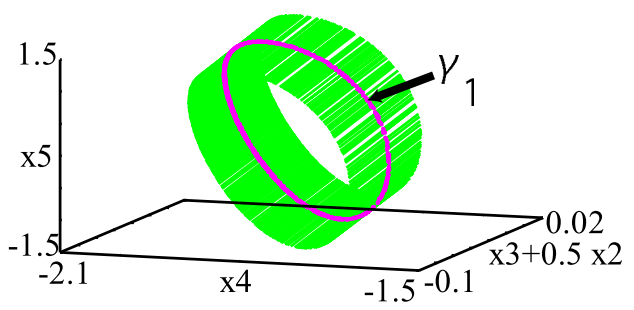

(e)

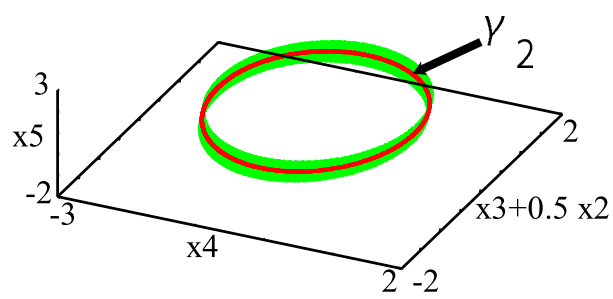

$(\mathrm{g})$

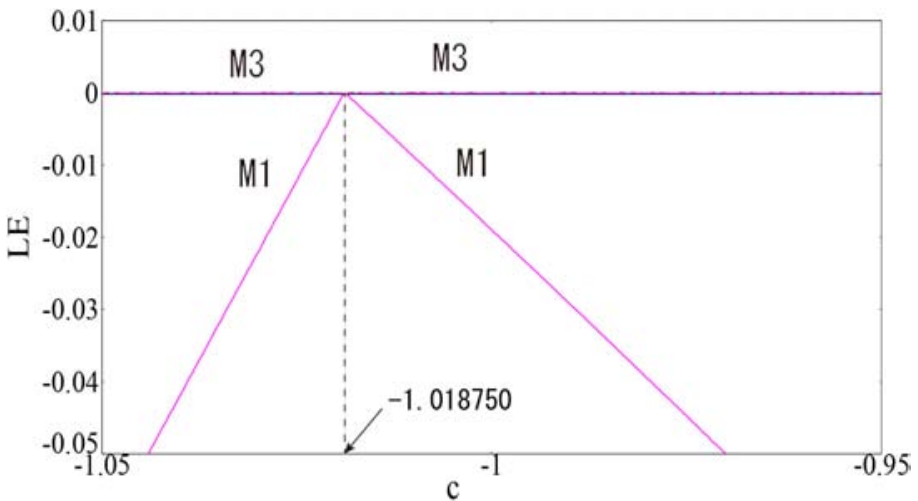

(b)

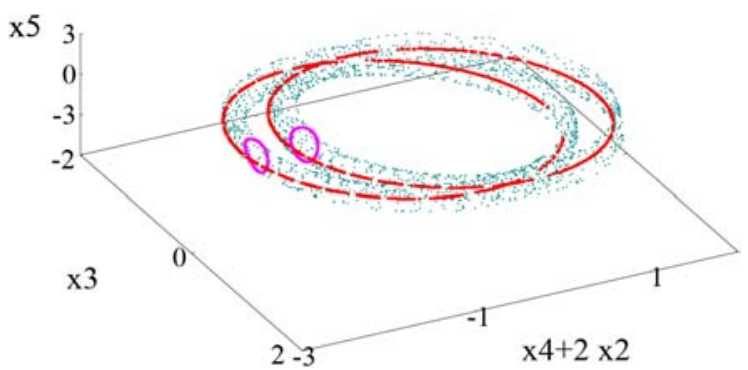

(d)

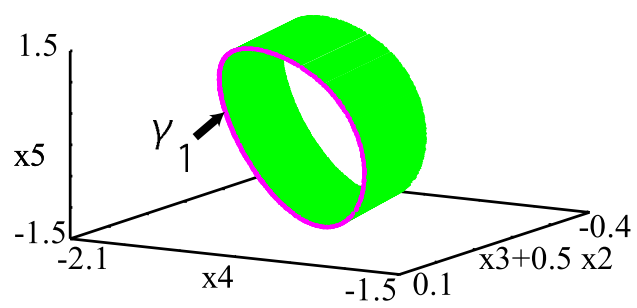

(f)

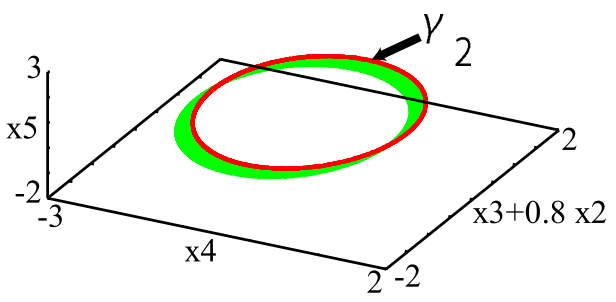

(h)

Fig. 19. MT3PD bifurcations. (a) Two-parameter Lyapunov diagram for $-1.10 \leq c \leq-0.90$ and $70 \times \pi / 180 \leq \theta_{1} \leq$ $80 \times \pi / 180$. The green, red, and blue denote regions of $\mathrm{M}(0)=4,3$ and 2, respectively. (b) One-parameter Lyapunov diagram for $-1.05 \leq c \leq-0.95$ at $\theta_{1}=74 \times \pi / 180$. (c) ST2 on $\Sigma_{1}=\left\{x_{0}=0, x_{1}<0\right\}$ in $\left(x_{3}, x_{4}+2 x_{2}, x_{5}\right)$-space at $c=-1.0$, prior to the MT3PD bifurcation. The magenta circle $\gamma_{1}$ represents ST1 on a codimension two section $\Sigma_{2}=\left\{x_{0}=0, x_{1}<0, x_{3}=0, x_{4}<0\right\}$. The red circle $\gamma_{2}$ represents ST1 on a codimension two section $\Sigma_{3}=\left\{x_{0}=0, x_{1}<0, x_{5}=0, x_{6}<0\right\}$. (d) ST2 on $\Sigma_{1}$ in $\left(x_{3}, x_{4}+2 x_{2}, x_{5}\right)$-space at $c=-1.025$ after the MT3PD bifurcation. The magenta circles and the red circles represent twoperiodic ST1s on $\Sigma_{2}$ and $\Sigma_{3}$, respectively. (e) A type $A^{-}$DLB of $\gamma_{1}$ under the once-iterated map in $\left(x_{3}+0.5 x_{2}, x_{4}, x_{5}\right)$-space at $c=-1.0$. (f) A DLB of $\gamma_{1}$ under the twice-iterated map in $\left(x_{3}+0.5 x_{2}, x_{4}, x_{5}\right)$-space at $c=-1.0$. (g) A type $A^{-}$DLB of $\gamma_{2}$ under the once-iterated map in $\left(x_{3}+0.5 x_{2}, x_{4}, x_{5}\right)$-space at $c=-1.0$. (h) A DLB of $\gamma_{2}$ under the twice-iterated map in $\left(x_{3}+0.8 x_{2}, x_{4}, x_{5}\right)$-space at $c=-1.0$. The slice radii are 0.01 for $(\mathrm{g})$ and $(\mathrm{h})$. 
two section $\Sigma_{3}=\left\{x_{0}=0, x_{1}<0, x_{5}=0, x_{6}<0\right\}$. Figures 19(e) and 19(f) present DLBs of $\gamma_{1}$ under the once-iterated map and the twice-iterated map, respectively, at $c=-1.0$. The former is an annulus with two sides, and the latter is an annulus with one side. Hence, the DLB of $\gamma_{1}$ is of type $A^{-}$. Similarly, Figs. 19(g) and 19(h) present DLBs of $\gamma_{2}$ under the once-iterated map and the twice-iterated map, respectively, at $c=-1.0$. The former is an annulus with two sides, and the latter is an annulus with one side. Hence, the DLB of $\gamma_{2}$ is also of type $A^{-}$. Figure $19(\mathrm{~d})$ presents a two-periodic
ST2 on $\Sigma_{1}$ in $\left(x_{3}, x_{4}+2 x_{2}, x_{5}\right)$-space at $c=-1.025$ after the MT3PD bifurcation. The magenta and red circles represent two-periodic ST1s on $\Sigma_{2}$ and $\Sigma_{3}$, respectively.

From these results, we observe the ST1PD bifurcations of $\gamma_{1}$ and $\gamma_{2}$. Hence, the MT3PD bifurcation occurs.

\subsection{Double covering bifurcation of MT3 $(M T 3 D C)$}

We present an example of MT3DC. Consider the following discrete-time dynamical system on $\mathbb{R}^{7}$ :

$$
\left\{\begin{aligned}
x_{0}(t+1)= & r_{1} \cos \theta_{1} x_{0}(t)-r_{1} \sin \theta_{1} x_{1}(t)-\left(r_{1}-0.9\right)\left(x_{0}^{2}(t)+x_{1}^{2}(t)\right) \\
& +e_{1} x_{2}(t)+e_{2} x_{3}(t)+e_{3} x_{5}(t), \\
x_{1}(t+1)= & r_{1} \sin \theta_{1} x_{0}(t)+r_{1} \cos \theta_{1} x_{1}(t)-\left(r_{1}-0.9\right)\left(x_{0}^{2}(t)+x_{1}^{2}(t)\right), \\
x_{2}(t+1)= & x_{0}(t), \\
x_{3}(t+1)= & r_{2} \cos \theta_{2} x_{3}(t)-r_{2} \sin \theta_{2} x_{4}(t)-\left(r_{2}-0.9\right)\left(x_{3}^{2}(t)+x_{4}^{2}(t)\right), \\
x_{4}(t+1)= & r_{2} \sin \theta_{2} x_{3}(t)+r_{2} \cos \theta_{2} x_{4}(t)-\left(r_{2}-0.9\right)\left(x_{3}^{2}(t)+x_{4}^{2}(t)\right), \\
x_{5}(t+1)= & r_{3} \cos \theta_{3} x_{5}(t)-r_{3} \sin \theta_{3} x_{6}(t)-\left(r_{3}-0.9\right)\left(x_{5}^{2}(t)+x_{6}^{2}(t)\right), \\
x_{6}(t+1)= & r_{3} \sin \theta_{3} x_{5}(t)+r_{3} \cos \theta_{3} x_{6}(t)-\left(r_{3}-0.9\right)\left(x_{5}^{2}(t)+x_{6}^{2}(t)\right),
\end{aligned}\right.
$$

where $r_{1}$ and $\theta_{1}$ are the bifurcation parameters, and the other parameters are fixed as $r_{2}=1.02, e_{1}=-0.21$, $e_{2}=0.01, e_{3}=0.01, r_{3}=1.02$, and $\theta_{3}=73.2 \times \pi / 180$.

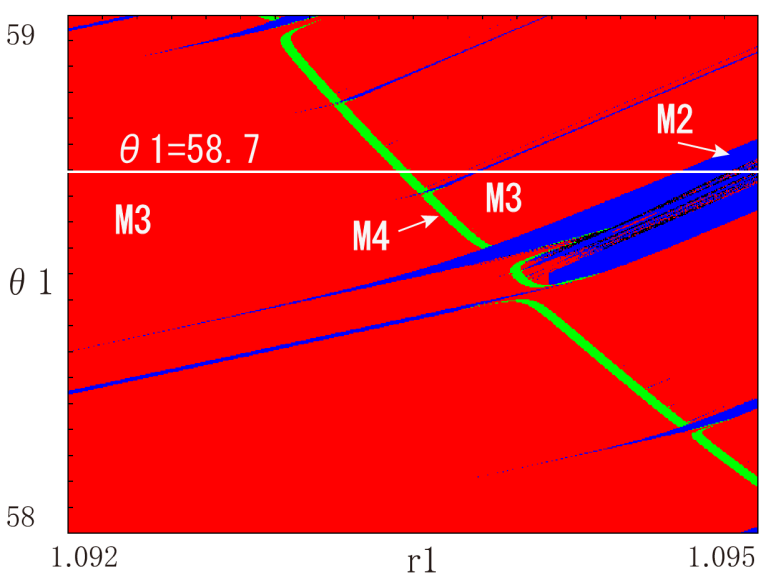

(a)

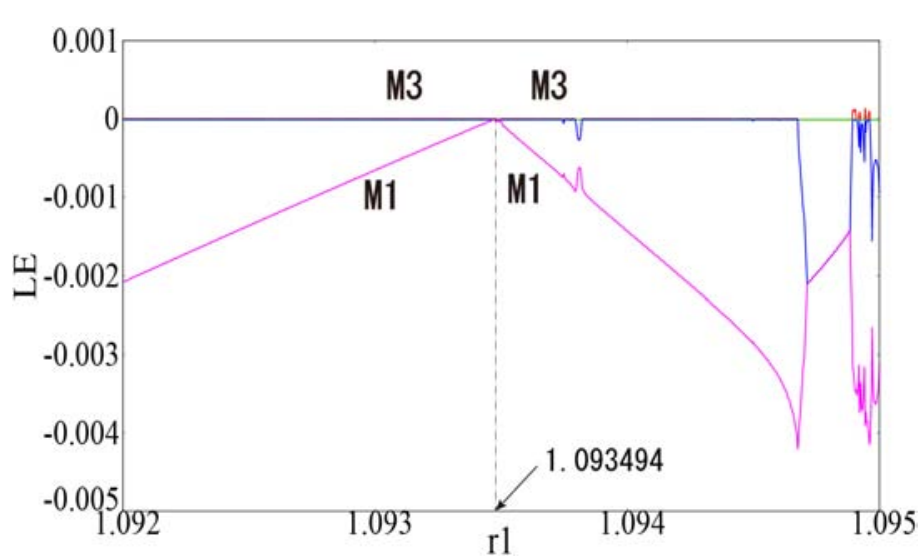

(b)

Fig. 20. MT3DC bifurcations. (a) Two-parameter Lyapunov diagram. The green, red, and blue denote regions of $\mathrm{M}(0)=4$, 3, and 2, respectively. (b) One-parameter Lyapunov diagram for $1.092 \leq r_{1} \leq 1.095$ at $\theta_{1}=58.7 \times \pi / 180$. (c) ST2 on $\Sigma_{1}=\left\{x_{3}=0, x_{4}>0\right\}$ in $\left(x_{0}, x_{1}, x_{5}+2 x_{2}\right)$-space at $r_{1}=1.093$, prior to the MT3DC bifurcation. The red circle $\gamma_{1}$ represents ST1 on $\Sigma_{2}=\left\{x_{3}=0, x_{4}>0, x_{0}=0, x_{1}<0\right\}$. The magenta circle $\gamma_{2}$ represents ST1 on $\Sigma_{3}=\left\{x_{3}=0, x_{4}>0, x_{5}=0, x_{6}>0\right\}$. (d) ST2 on $\Sigma_{1}$ on $\left(x_{0}, x_{1}, x_{5}+2 x_{2}\right)$-space at $r_{1}=1.094$ after the MT3DC bifurcation. The red circles represent double component ST1s on $\Sigma_{2}$. The magenta circle represents a double covering ST1 on $\Sigma_{3}$. (e) A type $A^{*}$ DLB of $\gamma_{1}$ under the once-iterated map at $r_{1}=1.093$. (f) A type $A^{*}$ DLB of $\gamma_{1}$ under the twice-iterated map at $r_{1}=1.093$. (g) A type $M$ DLB of $\gamma_{2}$ under the once-iterated map at $r_{1}=1.093$. (h) A type $M$ DLB of $\gamma_{2}$ under the twice-iterated map at $r_{1}=1.093$. The slice radii are 0.01 for (e)-(h). 


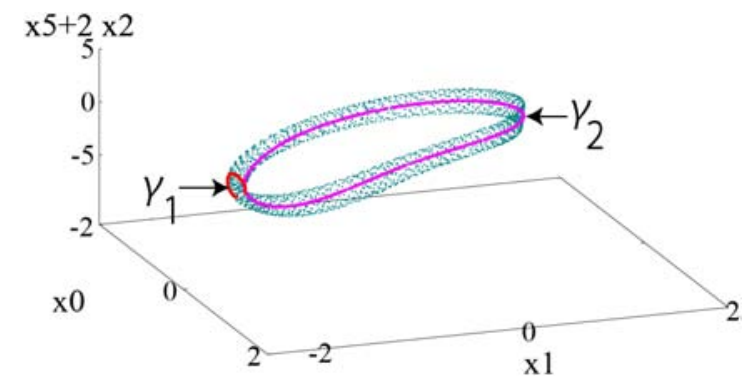

(c)

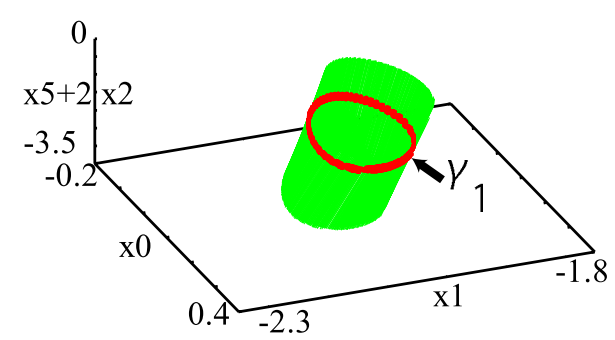

(e)

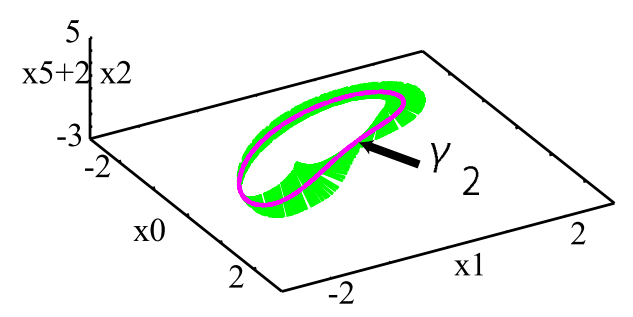

(g)

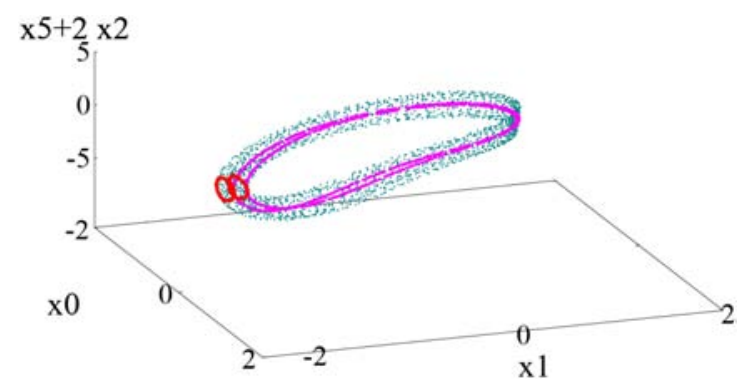

(d)

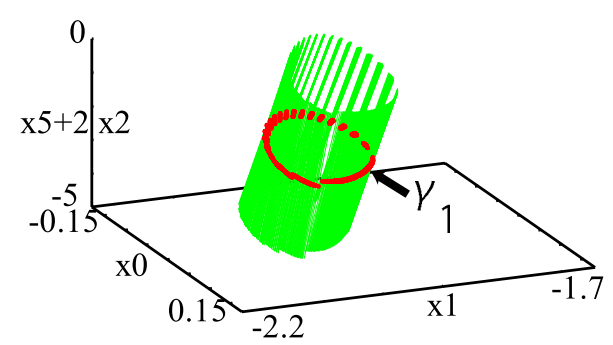

(f)

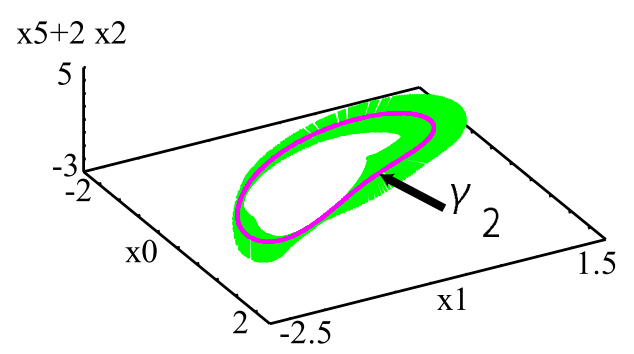

(h)

Fig. 20. (Continued)

Figure 20(a) presents a two-parameter Lyapunov diagram of Eqs. (18) for $1.092 \leq r_{1} \leq 1.095$ and $58 \times \pi / 180 \leq \theta_{1} \leq 59 \times \pi / 180$. The green, red and blue regions denote $\mathrm{M}(0)=4,3$ and 2 , respectively. The MT3DC bifurcation set is approximately denoted by the green region, because the multiplicity of the zero LE M(0) increases from three to four at the MT3DC bifurcation points. The white line represents the domain of the one-parameter Lyapunov diagram in Fig. 20(b) for $1.092 \leq r_{1} \leq 1.095$ at $\theta_{1}=58.7 \times \pi / 180$. We observe that the DLE touches zero at $r_{1}=1.093494$. Figure 20(c) presents ST2 on a codimension one section $\Sigma_{1}=\left\{x_{3}=0\right.$, $\left.x_{4}>0\right\}$ in $\left(x_{0}, x_{1}, x_{5}+2 x_{2}\right)$-space at $r_{1}=1.093$, prior to the MT3DC bifurcation. The red circle $\gamma_{1}$ and the magenta circle $\gamma_{2}$ represent ST1s on the codimension two sections $\Sigma_{2}=\left\{x_{3}=0, x_{4}>0\right.$, $\left.x_{0}=0, x_{1}<0\right\}$ and $\Sigma_{3}=\left\{x_{3}=0, x_{4}>0, x_{5}=0\right.$, $\left.x_{6}>0\right\}$, respectively. Figures 20(e) and 20(f) present DLBs of $\gamma_{1}$ under the once-iterated map and the twice-iterated map, respectively, at $r_{1}=1.093$. Both the former and the latter are annuli with two sides. Hence, the DLB of $\gamma_{1}$ is of type $A^{*}$. Similarly, Figs. 20(g) and 20(h) present DLBs of $\gamma_{2}$ under the once-iterated map and the twice-iterated map, respectively, at $r_{1}=1.093$. Both the former and the latter are Möbius bands. Hence, the DLB of $\gamma_{2}$ is of type $M$. Figure 20(d) presents ST2 on $\Sigma_{1}$ in $\left(x_{0}, x_{1}, x_{5}+2 x_{2}\right)$-space at $r_{1}=$ 1.094 after the MT3DC bifurcation. The red circles represent double component ST1s on $\Sigma_{2}$. The magenta circle represents a double covering ST1 on $\Sigma_{3}$.

From these results, we observe the ST1CD bifurcation of $\gamma_{1}$ and the ST1DC bifurcation of $\gamma_{2}$. Hence, the MT3DC bifurcation occurs. 


\subsection{Neimark-Sacker bifurcation of MT3 (MT3NS)}

We present an example of MT3NS. Consider the following discrete-time dynamical system on $\mathbb{R}^{8}$ :

$$
\left\{\begin{aligned}
x_{0}(t+1)= & r_{1} \cos \theta_{1} x_{0}(t)-r_{1} \sin \theta_{1} x_{1}(t)-\left(r_{1}-0.9\right)\left(x_{0}^{2}(t)+x_{1}^{2}(t)\right)+e_{1} x_{2}(t), \\
x_{1}(t+1)= & r_{1} \sin \theta_{1} x_{0}(t)+r_{1} \cos \theta_{1} x_{1}(t)-\left(r_{1}-0.9\right)\left(x_{0}^{2}(t)+x_{1}^{2}(t)\right) \\
x_{2}(t+1)= & r_{2} \cos \theta_{2} x_{2}(t)-r_{2} \sin \theta_{2} x_{3}(t)-\left(r_{2}-0.9\right)\left(x_{2}^{2}(t)+x_{3}^{2}(t)\right) \\
& +e_{2} x_{0}(t)+e_{3} x_{4}(t)+e_{4} x_{6}(t) \\
x_{3}(t+1)= & r_{2} \sin \theta_{2} x_{2}(t)+r_{2} \cos \theta_{2} x_{3}(t)-\left(r_{2}-0.9\right)\left(x_{2}^{2}(t)+x_{3}^{2}(t)\right) \\
x_{4}(t+1)= & r_{3} \cos \theta_{3} x_{4}(t)-r_{3} \sin \theta_{3} x_{5}(t)-\left(r_{3}-0.9\right)\left(x_{4}^{2}(t)+x_{5}^{2}(t)\right) \\
x_{5}(t+1)= & r_{3} \sin \theta_{3} x_{4}(t)+r_{3} \cos \theta_{3} x_{5}(t)-\left(r_{3}-0.9\right)\left(x_{4}^{2}(t)+x_{5}^{2}(t)\right) \\
x_{6}(t+1)= & r_{4} \cos \theta_{4} x_{6}(t)-r_{4} \sin \theta_{4} x_{7}(t)-\left(r_{4}-0.9\right)\left(x_{6}^{2}(t)+x_{7}^{2}(t)\right) \\
x_{7}(t+1)= & r_{4} \sin \theta_{4} x_{6}(t)+r_{4} \cos \theta_{4} x_{7}(t)-\left(r_{4}-0.9\right)\left(x_{6}^{2}(t)+x_{7}^{2}(t)\right)
\end{aligned}\right.
$$

where $r_{2}$ and $\theta_{2}$ are the bifurcation parameters, and the other parameters are fixed as $r_{1}=1.0146$, $\theta_{1}=59.2812 \times \pi / 180, e_{1}=0.02, e_{2}=0.02$, $e_{3}=0.10, r_{3}=1.04, \theta_{3}=58.7 \times \pi / 180, r_{4}=1.04$, and $\theta_{4}=68.7 \times \pi / 180$.

Figure 21(a) presents a two-parameter Lyapunov diagram of Eqs. (19) for $75 \times \pi / 180 \leq \theta_{2} \leq$ $80 \times \pi / 180$ and $0.99 \leq r_{2} \leq 1.01$. The blue, green, and red regions denote $\mathrm{M}(0)=5,4$, and 3 , respectively. The MT3NS bifurcation set is approximately denoted by the blue line, because the multiplicity of zero LE M(0) increases from three to five at the MT3NS bifurcation points. The white line represents the domain of the one-parameter Lyapunov diagram in Fig. 21(b) for $0.99 \leq r_{2} \leq 1.01$ at $\theta_{2}=$ $72.1 \times \pi / 180$. We observe that the DLE touches zero at $r_{2}=1.002750$. Figure 21(c) presents ST2 on a codimension one section $\Sigma_{1}=\left\{x_{0}=0, x_{1}<0\right\}$ in $\left(x_{2}, x_{3} x_{4}\right)$-space at $r_{2}=0.9900$, prior to the MT3NS bifurcation. The magenta circle $\gamma$ represents ST1 on a codimension two section $\Sigma_{2}=\left\{x_{0}=0, x_{1}<\right.$ $\left.0, x_{2}=0, x_{3}>0\right\}$. Figure 21(e) presents a DLB of $\gamma$ in $\left(x_{2}, x_{3} x_{4}\right)$-space at $r_{2}=0.9900$, prior to the MT3NS bifurcation. This is of type $F$. Figure 21(d) presents ST3 on the codimension one section $\Sigma_{1}$ in $\left(x_{2}, x_{3} x_{4}\right)$-space at $r_{2}=1.01$ after the MT3NS bifurcation. The magenta two-torus presents ST2 on $\Sigma_{2}$.

From these results, we observe the ST1NS bifurcation. Hence, the MT3NS bifurcation occurs.

\subsection{Saddle-node cycle and homoclinic cycle bifurcations of MTO (MTOSNC/MTOHCC)}

In this subsection, we present an example of MTOSNC and MTOHCC. Consider the following discrete-time dynamical system on $\mathbb{R}^{2} / \mathbb{Z}^{2}$ :

$$
\left\{\begin{aligned}
x_{0}(t+1)= & x_{0}(t)+\frac{a_{1}}{360}+b_{1} \sin \left(2 \pi\left(x_{0}(t)-c_{1}\right)\right) \\
& +e_{1} \sin \left(2 \pi x_{1}(t)\right) \bmod 1, \\
x_{1}(t+1)= & x_{1}(t)+\frac{a_{2}}{360}+b_{2} \sin \left(2 \pi\left(x_{1}(t)-c_{2}\right)\right) \\
& +e_{2} \sin \left(2 \pi x_{0}(t)\right) \bmod 1,
\end{aligned}\right.
$$

where $a_{1}$ and $a_{2}$ are the bifurcation parameters, and the other parameters are fixed as $b_{1}=0.05, b_{2}=$ $0.05, c_{1}=0.25, c_{2}=0.25, e_{1}=0.02$, and $e_{2}=0.02$.

The center of Fig. 22 presents a two-parameter Lyapunov diagram of Eqs. (20) for $22.0 \leq a_{1} \leq 24.0$ and $17.5 \leq a_{2} \leq 20.5$. The magenta region (1) denotes $\mathrm{M}(0)=1$ where a stable MT1(s-MT1) and an unstable MT1(u-MT1) coexist. The yellow region (5) denotes $\mathrm{M}(0)=0$, where a stable MT0(s-MT0) and a saddle MT0(u-MT0) coexist. The orange denotes a region (3) in which s-MT0, u-MT0, s-MT1, and u-MT1 coexist. ${ }^{13}$

\footnotetext{
${ }^{13}$ In this region we observe a hysteresis of phase locking, because two attractors coexist.
} 


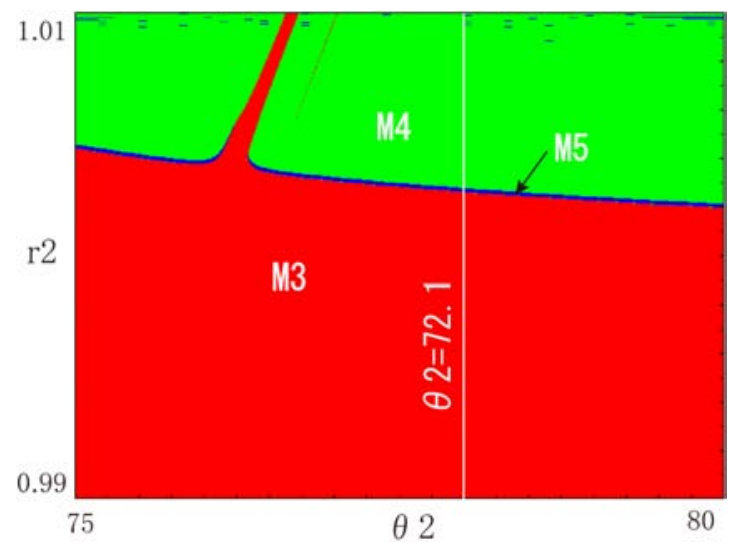

(a)

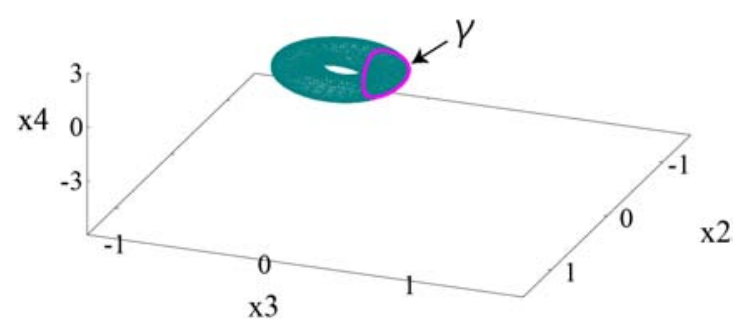

(c)

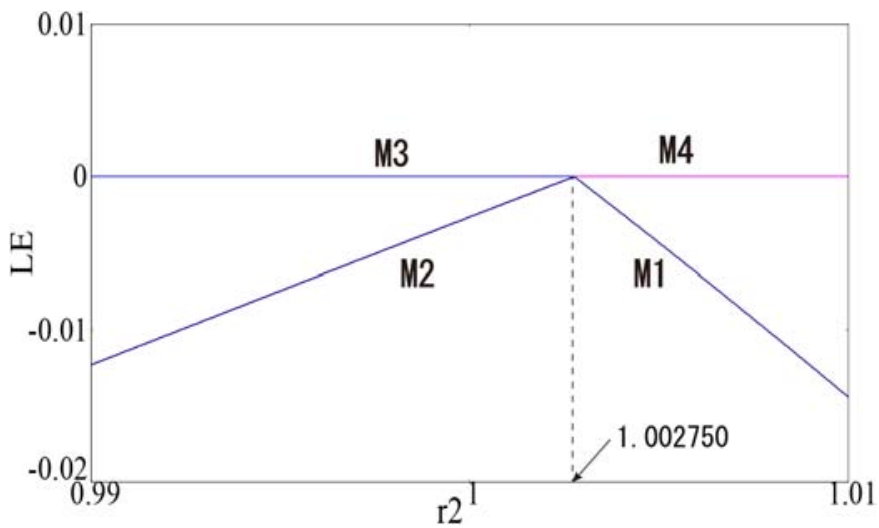

(b)

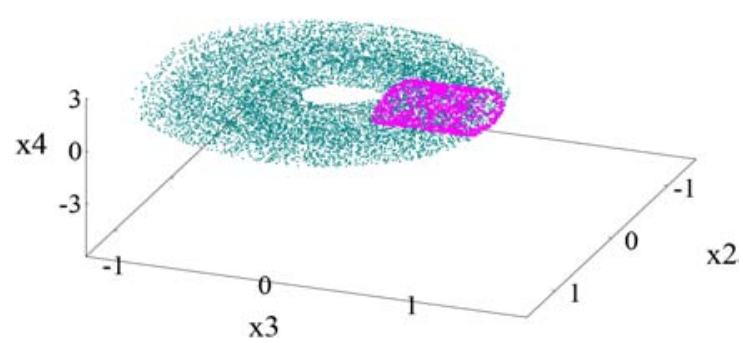

(d)

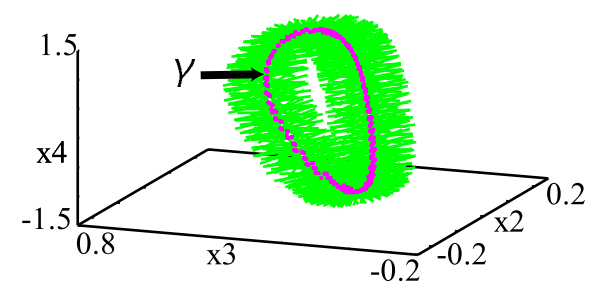

(e)

Fig. 21. MT3NS bifurcations. (a) Two-parameter Lyapunov diagram for $75 \times \pi / 180 \leq \theta_{2} \leq 80 \times \pi / 180$ and $0.99 \leq r_{2} \leq 1.01$. The blue, green and red denote regions of $\mathrm{M}(0)=5,4$ and 3, respectively. (b) One-parameter Lyapunov diagram of Eqs. (19) for $0.99 \leq r_{2} \leq 1.01$. (c) ST2 on a codimension one section $\Sigma_{1}=\left\{x_{0}=0, x_{1}<0\right\}$ in $\left(x_{2}, x_{3} x_{4}\right)$-space at $r_{2}=0.9900$. The magenta circle $\gamma$ represents ST1 on a codimension two section $\Sigma_{2}=\left\{x_{0}=0, x_{1}<0, x_{2}=0, x_{3}>0\right\}$. (d) ST3 on a codimension one section $\Sigma_{1}=\left\{x_{0}=0, x_{1}<0\right\}$ in $\left(x_{2}, x_{3} x_{4}\right)$-space at $r_{2}=1.01$. The magenta two-torus represents ST2 on a codimension two section $\Sigma_{2}=\left\{x_{0}=0, x_{1}<0, x_{2}=0, x_{3}>0\right\}$. (e) A type $F$ DLB of $\gamma$ at $r_{2}=0.9900$. The slice radius is 0.01 for $(\mathrm{e})$.

On the boundary (2) between the magenta region (1) and the orange region (3), an MTOSN bifurcation occurs; that is, s-MT0 and u-MT0 in the orange region collide at the boundary (2), and disappear in the magenta region. On the boundary (6) between the magenta region (1) and the yellow region (5), an MTOSNC bifurcation occurs; that is, s-MT0 and u-MT0 in the yellow region form a saddle-node homoclinic cycle at the boundary (6), and s-MT1 is created in the magenta region. On the boundary (4) between the orange region (3) and the yellow region (5), an MTOHCC bifurcation occurs; that is, the stable set and the unstable set of $\mathrm{u}$ MT0 form a homoclinic cycle, and s-MT1 is created in the orange region.

In panels (1)-(6) on the right and left sides of Fig. 22, several orbits are demonstrated in $\left(x_{0}, x_{1}\right)$ space, where $-0.5 \leq x_{0} \leq 0.5,-0.5 \leq x_{1} \leq 0.5 .^{14}$

\footnotetext{
${ }^{14}$ The right edges and left edges are identified, and the top edges and bottom edges are also identified, as a two-dimensional torus.
} 


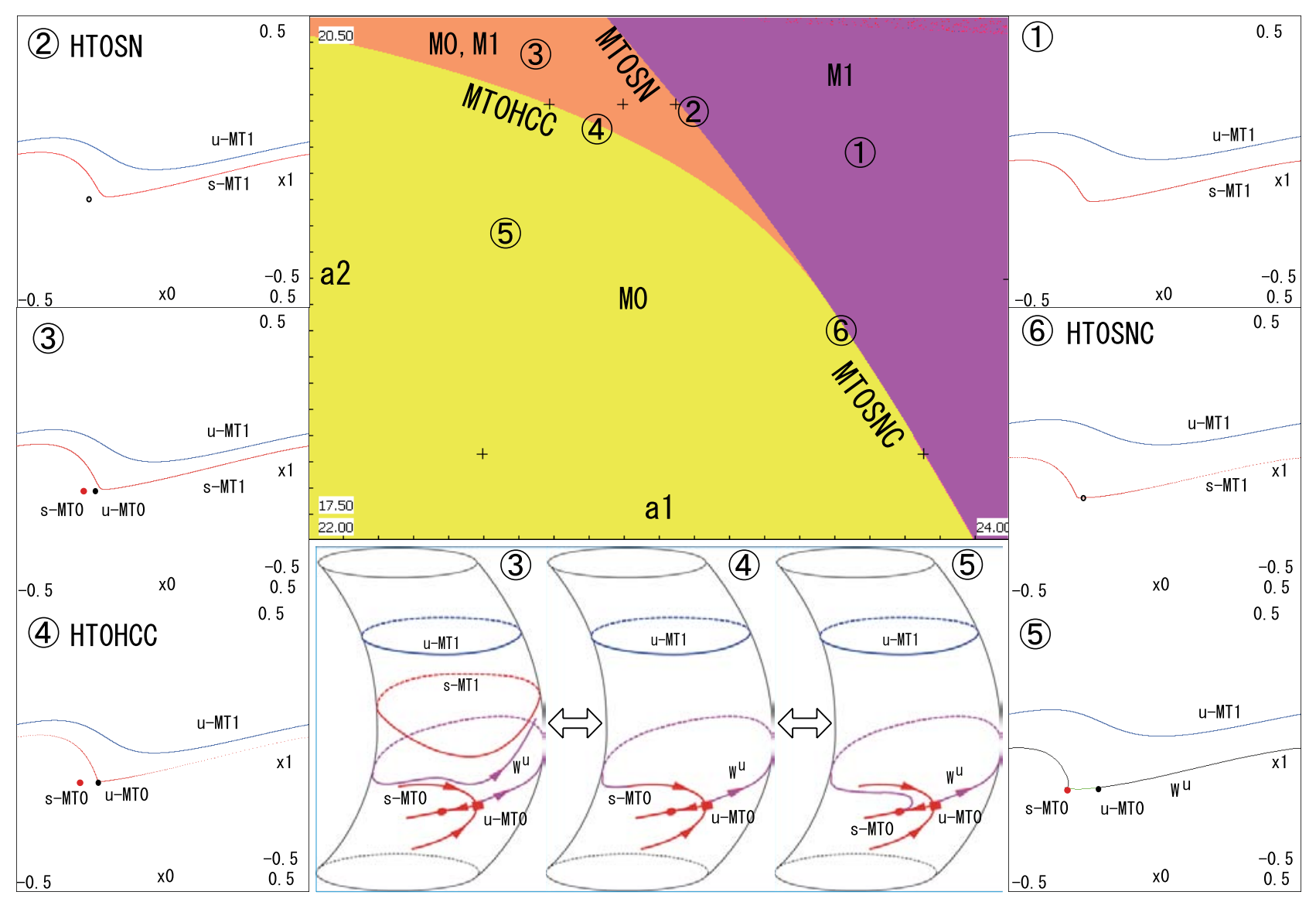

Fig. 22. MTOSNC and MTOHCC bifurcations. Center: Two-parameter Lyapunov diagram. The magenta and yellow denote regions of $\mathrm{M}(0)=1,0$, respectively. The orange denotes a region in which s-MT0, u-MT0, s-MT1, and u-MT1 coexist. Right and left: Orbits on $\left(x_{0}, x_{1}\right)$-space. Bottom: An illustration of the MT0HCC bifurcation with the corresponding panels (3)-(5).

The values of $\left(a_{1}, a_{2}\right)$ are given by $(24.0,19.0)$ for (1), $(23.05,20.0)$ for (2), $(22.9,20.0)$ for (3), $(22.69,20.0)$ for (4), $(22.5,18.0)$ for (5), and $(23.77,18.0)$ for (6). The red and blue curves represent s-MT1 and u-MT1, respectively. ${ }^{15}$ The red and black dots represent s-MT0 and u-MT0, respectively. In panel (5), two curves from u-MT0 denote the unstable sets of the u-MT0, which form a homoclinic cycle. The open circle in panels (2) and (6) denotes a position where s-MT0 and u-MT0 collided.

The bottom of Fig. 22 presents an illustration of the MT0HCC bifurcation with the corresponding panels (3)-(5).

\subsection{Saddle-node cycle and homoclinic cycle bifurcations of MT1 (MT1SNC/MT1HCC)}

We next present an example of MT1SNC and MT1HCC. Consider the following discrete-time dynamical system on $\mathbb{R}^{3} / \mathbb{Z}^{3}$ :

$$
\left\{\begin{array}{l}
x_{0}(t+1)=x_{0}(t)+\frac{a_{1}}{360}+b_{1} \sin \left(2 \pi\left(x_{0}(t)-c_{1}\right)\right)+e_{12} \sin \left(2 \pi x_{1}(t)\right)+e_{13} \sin \left(2 \pi x_{2}(t)\right) \quad \bmod 1, \\
x_{1}(t+1)=x_{1}(t)+\frac{a_{2}}{360}+b_{2} \sin \left(2 \pi\left(x_{1}(t)-c_{2}\right)\right)+e_{23} \sin \left(2 \pi x_{2}(t)\right)+e_{21} \sin \left(2 \pi x_{0}(t)\right) \quad \bmod 1, \\
x_{2}(t+1)=x_{2}(t)+\frac{a_{3}}{360}+b_{3} \sin \left(2 \pi\left(x_{2}(t)-c_{3}\right)\right)+e_{31} \sin \left(2 \pi x_{0}(t)\right)+e_{32} \sin \left(2 \pi x_{1}(t)\right) \quad \bmod 1,
\end{array}\right.
$$

\footnotetext{
${ }^{15}$ The orbit of u-MT1 is calculated by inverse mapping using the Newton method.
} 

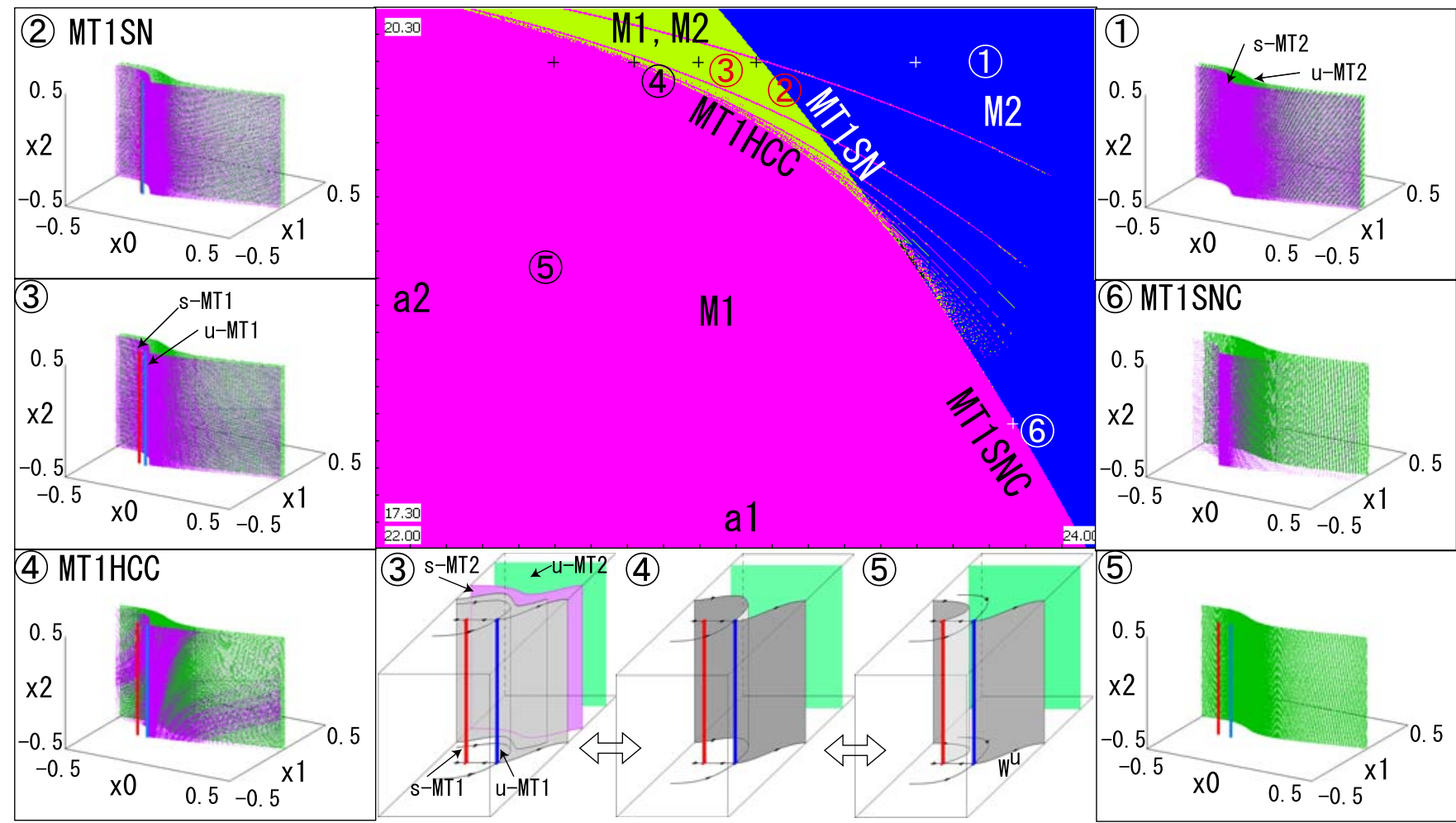

Fig. 23. MT1SNC and MT1HCC bifurcations. Center: Two-parameter Lyapunov diagram. The blue and magenta denote regions of $\mathrm{M}(0)=2,1$, respectively. The green denotes a region in which s-MT1, u-MT1, s-MT2, and u-MT2 coexist. Right and left: Orbits in $\left(x_{0}, x_{1}, x_{2}\right)$-space. Bottom: An illustration of the MT1HCC bifurcation with the corresponding panels (3)-(5).

where $a_{1}$ and $a_{2}$ are the bifurcation parameters, and the other parameters are fixed as $b_{1}=0.05$, $c_{1}=0.0, e_{12}=0.02, e_{13}=0.0001, b_{2}=0.05$ $c_{2}=0.25, e_{21}=0.0, e_{23}=0.02, a_{3}=25.0$ $b_{3}=0.01, c_{3}=0.0, e_{31}=0.0$, and $e_{32}=0.001$.

The center of Fig. 23 presents a two-parameter Lyapunov diagram of Eqs. (21) for $22.0 \leq a_{1} \leq$ 24.0 and $17.3 \leq a_{2} \leq 20.3$. The blue region (1) denotes $\mathrm{M}(0)=2$, where a stable MT2(s-MT2) and an unstable MT2(u-MT2) coexist. The magenta region (5) denotes $\mathrm{M}(0)=1$, where a stable MT1(sMT1) and a saddle MT1(u-MT1) coexist. The green denotes a region (3) in which s-MT1, u-MT1, sMT2, and u-MT2 coexist. ${ }^{16}$ On the boundary (2) between the blue region (1) and the green region (3), an MT1SN bifurcation occurs; that is, s-MT1 and $\mathrm{u}-\mathrm{MT} 1$ in the green region collide at the boundary (2), and disappear in the blue region. On the boundary (6) between the blue region (1) and the magenta region (5), an MT1SNC bifurcation occurs; that is, s-MT1 and u-MT1 in the magenta region form a saddle-node homoclinic cycle at the boundary (6), and s-MT2 is created. On the boundary (4) between the green region (3) and the magenta region (5), an MT1HCC bifurcation occurs; that is, the stable set and the unstable set of the uMT1 form a homoclinic cycle, and s-MT2 is created.

In panels (1) - (6) on the right and left sides of Fig. 23, several orbits are demonstrated in $\left(x_{0}, x_{1}, x_{2}\right)$-space where $-0.5 \leq x_{0} \leq 0.5,-0.5 \leq$ $x_{1} \leq 0.5$, and $-0.5 \leq x_{2} \leq 0.5 .{ }^{17}$

The values of $\left(a_{1}, a_{2}\right)$ are given by $(23.5,20.0)$ for (1), $(23.06,20.0)$ for (2), $(22.9,20.0)$ for (3), $(22.72$, $20.0)$ for (4), $(22.5,20.0)$ for (5), and $(23.77,18.0)$ for (6). The magenta and the green surfaces represent s-MT2 and u-MT2, respectively. ${ }^{18}$ The red and blue lines represent s-MT1 and u-MT1, respectively.

At the bottom of Fig. 23 is presented an illustration of the MT1HCC bifurcation with the corresponding panels (3)-(5).

\footnotetext{
${ }^{16}$ In this region we observe a hysteresis of phase locking, because two attractors coexist.

${ }^{17}$ The left and right, top and bottom, and front and back planes are identified, as a three-dimensional torus.

${ }^{18}$ The orbit of $\mathrm{u}-\mathrm{MT} 2$ is calculated by inverse mapping using the Newton method.
} 


\section{Bifurcations of FT3 in Continuous-Time Dynamical Systems}

In this section, we demonstrate bifurcations of FT3. In one-parameter Lyapunov diagrams, the $n$-fold multiplicity of both the DLE and zero LE is denoted by $\mathrm{M} n$. To obtain ST1 from FT3, we first take a Poincaré section of FT3, which is a codimension one section. Then, we obtain MT2 from the Poincaré map. Further, we apply a codimension one section to the MT2, which is a codimension two section for the FT3. Then, we obtain ST1. We calculate the DLB of MT2, to analyze the bifurcation mechanism. Throughout this section, MT2 is represented by blue, the two ST1s are represented by red and orange, respectively, and the DLB is represented by green. The radius of the slice corresponding to the codimension two section is fixed at 0.02 .

\subsection{Saddle-node bifurcation of FT3 (FT3SN)}

As an example of FT3SN, we adopt the phaselocked loop (PLL) circuit that has an input with a carrier frequency modulated by three incommensurate sinusoidal baseband signals. The circuit is shown in Fig. 24, where PC is a phase comparator, $F(s)$ is a single-pole low-pass filter, and $\mathrm{VCO}$ is a voltage controlled oscillator. The input $y_{1}$ can be denoted as $y_{1}=A \sin \left(\omega_{0} t+\theta_{i}(t)\right)$, where $\dot{\theta}_{i}(t)=\Delta \omega+p_{1} \sin \left(\omega_{1} t\right)+p_{2} \sin \left(\omega_{2} t\right)+p_{3} \sin \left(\omega_{3} t\right)$. The VCO output signal can be denoted as $y_{2}=$ $B \cos \left(\omega_{0} t+\theta_{o}(t)\right)$. By $\omega_{0}$, we denote the freerunning VCO frequency, and $\theta_{i}(t)$ and $\theta_{o}(t)$ are the input and output phases, respectively. We define a new variable $\phi(t)=\theta_{i}(t)-\theta_{o}(t)$, which is called the error phase. The dynamics of the PLL can be represented by using $\phi(t)$ as a state variable, as follows:

$$
\left\{\begin{aligned}
\dot{x}_{0}= & x_{1}, \\
\dot{x}_{1}= & -\beta x_{1}-\sin x_{0}+\beta \sigma+m_{1} \beta \sin x_{2} \\
& +m_{1} \omega_{1} \cos x_{2}+m_{2} \beta \sin x_{3}+m_{2} \omega_{2} \cos x_{3} \\
& +m_{3} \beta \sin x_{4}+m_{3} \omega_{3} \cos x_{4}, \\
\dot{x}_{2}= & \omega_{1} \\
\dot{x}_{3}= & \omega_{2}, \\
\dot{x}_{4}= & \omega_{3},
\end{aligned}\right.
$$

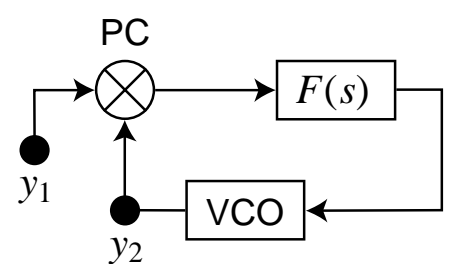

Fig. 24. The PLL circuit with three sinusoidal baseband signals.

where, $x_{0}=\phi \in \mathbb{S}^{1}, x_{1}=\dot{\phi} \in \mathbb{R}^{1}, x_{2}=\omega_{1} t \in \mathbb{S}^{1}$, $x_{3}=\omega_{2} t \in \mathbb{S}^{1}$, and $x_{4}=\omega_{3} t \in \mathbb{S}^{1}$. Therefore, the system is in the phase space of $\mathbb{S}^{1} \times \mathbb{R}^{1} \times \mathbb{S}^{1} \times \mathbb{S}^{1} \times \mathbb{S}^{1}$. Here $\mathbb{S}^{1}$ denotes a one-dimensional toroidal coordinate system, identifying $-\pi$ with $\pi$. The parameters $\beta$ and $\sigma$ denote the normalized angular frequency and detuning, respectively. The parameters $m_{i}$ and $\omega_{i}$ denote the $i$ th input amplitude and angular frequency, respectively, for $i=1,2,3$. In the following calculation, the parameter setting is based on the FT1 bifurcations shown in [Endo, 1990]. The bifurcation parameter is $\omega_{1}$, and the other parameters are fixed as $\beta=0.56, \sigma=1.28, m_{1}=0.5, m_{2}=$ $0.03, m_{3}=0.01, \omega_{2}=0.473$, and $\omega_{3}=0.21123$. In this system, two solutions, called Types 1 and 2, coexist for some parameter region. The values of $\left(x_{0}, x_{1}\right)$ for the Type 1 solution are bounded to a part of the surface of cylindrical phase space $\mathbb{S}^{1} \times \mathbb{R}^{1}$. In contrast, those of the Type 2 solution are unbounded; that is, the flow moves around the cylindrical surface $\mathbb{S}^{1} \times \mathbb{R}^{1}$. FT3SN occurs through the disappearance of a stable Type 2 solution (node) and its unstable counterpart (saddle). The saddle solution is sandwiched by stable Type 1 and stable Type 2 solutions. Figure 25 represents a oneparameter Lyapunov diagram of a Type 2 solution, demonstrating FT3SN calculated from Eqs. (22). Note that the slope of the DLE curve is perpendicular to the horizontal line at the bifurcation point of $\omega_{1}=1.44034$. This is a marked characteristic of the SN bifurcation. Figure 26 shows the MT2 (blue) on the Poincaré section $\Sigma_{1}=\left\{x_{2}=0, \dot{x}_{2}>0\right\}$, and the ST1 (red) on a codimension two section $\Sigma_{2}=\left\{x_{2}=0, \dot{x}_{2}>0, x_{4}=0\right\}$. Our simulation results show that after the FT3SN bifurcation, the Type 2 solution disappears, and the state jumps to the coexisting Type 1 solution. To clarify this bifurcation mechanism, we present the DLB before the bifurcation point at $\omega_{1}=1.44$ in Fig. 27. Because the DLB of this ST1 is of type $A^{+}$, the original DLB of the FT3 is of type $A^{3+}$. Hence, we observe the FT3SN bifurcation. 


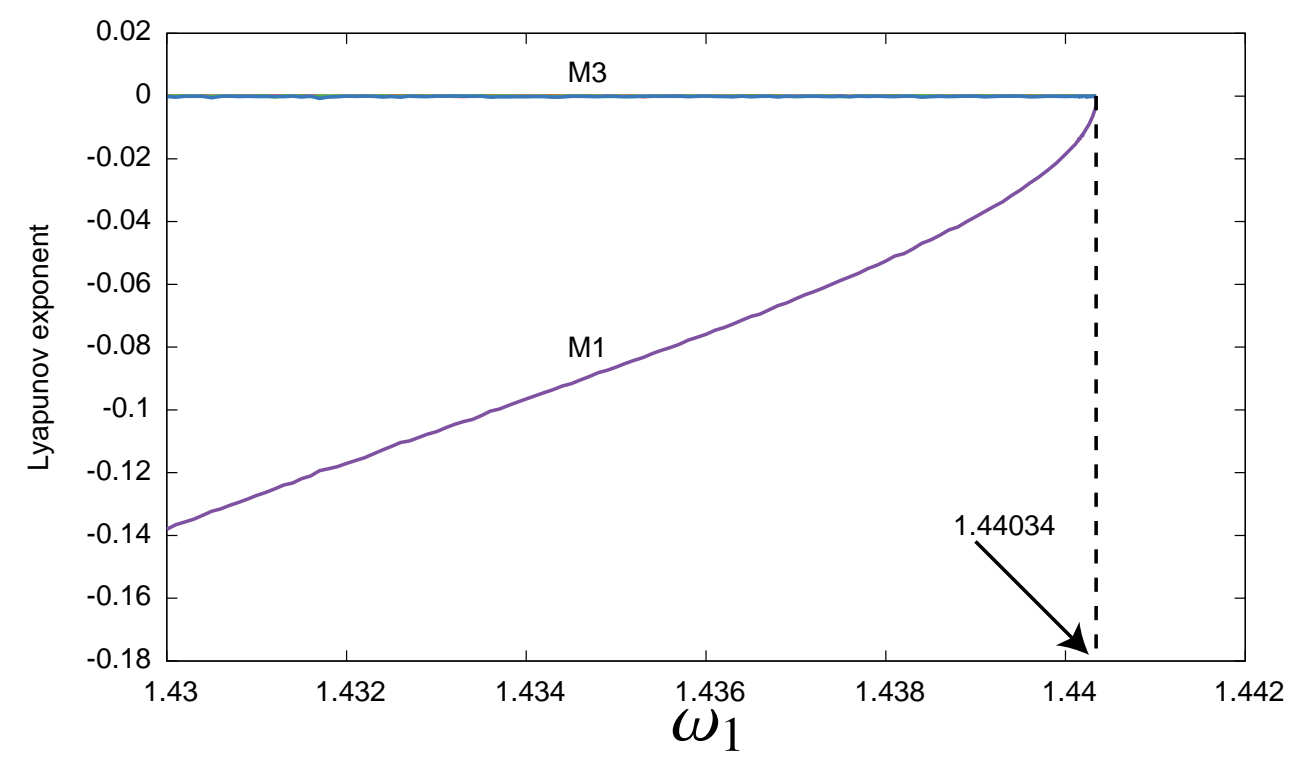

Fig. 25. One-parameter Lyapunov diagram of a Type 2 solution, in terms of $\omega_{1}$, demonstrating FT3SN at $\omega_{1}=1.44034$.

\subsection{Double covering bifurcation of FT3 (FT3DC)}

Next, as an example of FT3DC, we consider the same PLL system as Eqs. (22). In the following calculation, the bifurcation parameter is $\omega_{1}$, and the other parameters are fixed as $\beta=0.56$, $\sigma=1.28, m_{1}=0.05, m_{2}=0.03, m_{3}=0.01$, $\omega_{2}=0.33123$, and $\omega_{3}=0.21123$. Figure 28 presents a one-parameter Lyapunov diagram of the Type 1 solution in terms of $\omega_{1}$. The DLE touches zero at $\omega_{1}=1.2817$, and the multiplicity of the zero LE before the bifurcation is three. Therefore, a local bifurcation of FT3 occurs at this point.

Figure 29 shows MT2 (a) before and (b) after the bifurcation. A local bifurcation of the MT2 appears to have occurred. However, the bifurcation type is not clear. Figure 30 shows ST1 on $\Sigma_{2}$ (a) before and (b) after the bifurcation. We can infer

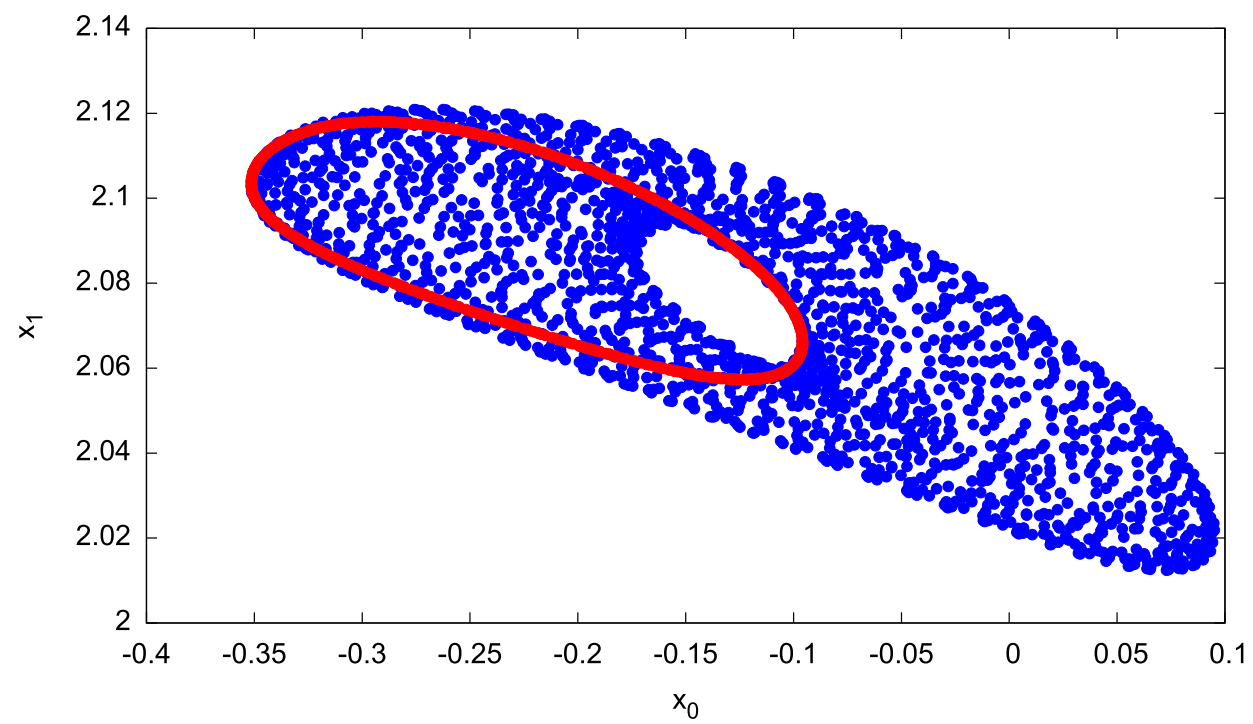

Fig. 26. MT2 for the Type 2 solution on the Poincaré section $\Sigma_{1}=\left\{x_{2}=0, \dot{x}_{2}>0\right\}$, and the ST1 on the codimension two section $\Sigma_{2}=\left\{x_{2}=0, \dot{x}_{2}>0, x_{4}=0\right\}$, prior to the FT3SN. The blue points represent the MT2 and the red points represent the ST1. 


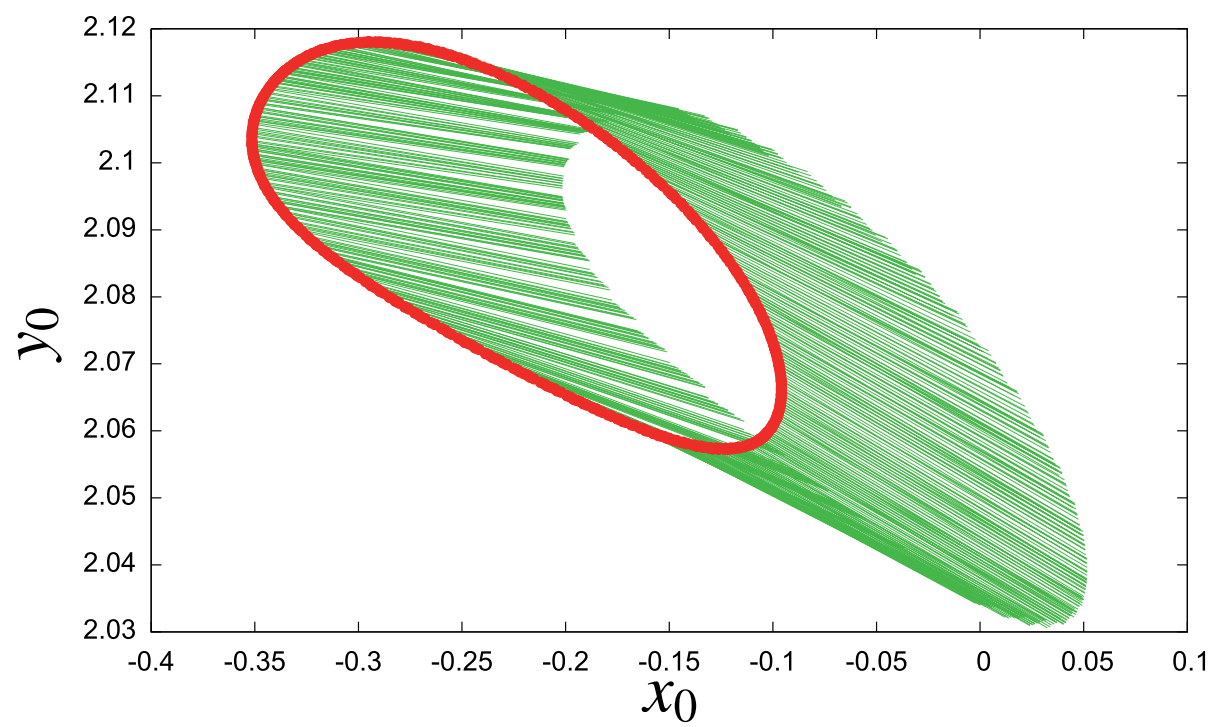

Fig. 27. The type $A^{+}$DLB of the ST1 for the Type 2 solution on the section $\Sigma_{2}=\left\{x_{2}=0, \dot{x}_{2}>0, x_{4}=0\right\}$, prior to the FT3SN. The red points represent the ST1, and the green lines represent its corresponding DLB.

that a one-turn ST1 bifurcates to two one-turn ST1s via ST1CD. Figure 31 shows ST1 on $\Sigma_{3}$ (a) before and (b) after the bifurcation. We can infer that a one-turn ST1 bifurcates to a two-turn ST1 via ST1DC. In order to illuminate the mechanism of bifurcation of FT3, we calculate the DLB of the ST1 before the bifurcation at $\omega_{1}=1.284$. Figure 32 represents the DLB of the ST1 on (a) $\Sigma_{2}$ and (b) $\Sigma_{3}$ before the bifurcation at $\omega_{1}=1.284$. The DLB in Fig. 32(a) is of type $A^{*}$. Therefore, an ST1CD bifurcation occurs on $\Sigma_{2}$. In contrast, the DLB in Fig. 32(b) is of type $M$. Therefore, an ST1DC bifurcation occurs on $\Sigma_{3}$. Summarizing these results, the original DLB of FT3 is of type $M^{3}$. Hence, the FT3DC bifurcation occurs at $\omega_{1}=$ 1.2817 in Fig. 28.

\subsection{Neimark-Sacker bifurcation of FT3 (FT3NS)}

As an example of FT3NS, we present a ladder of four-coupled van der Pol (abbr. VDP) oscillators. Figure 33 shows a circuit model of four-coupled VDP oscillators coupled by registers with large frequency detuning.

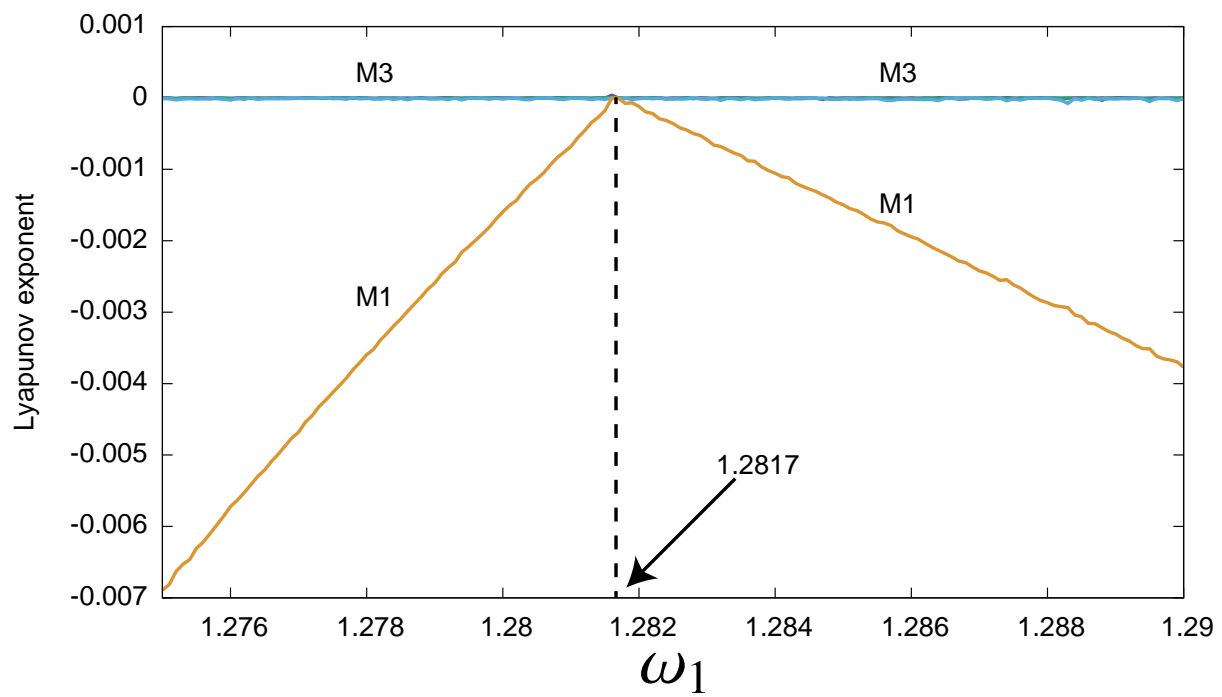

Fig. 28. One-parameter Lyapunov diagram of Type 1 solution in terms of $\omega_{1}$, demonstrating FT3DC. 
M. Komuro et al.

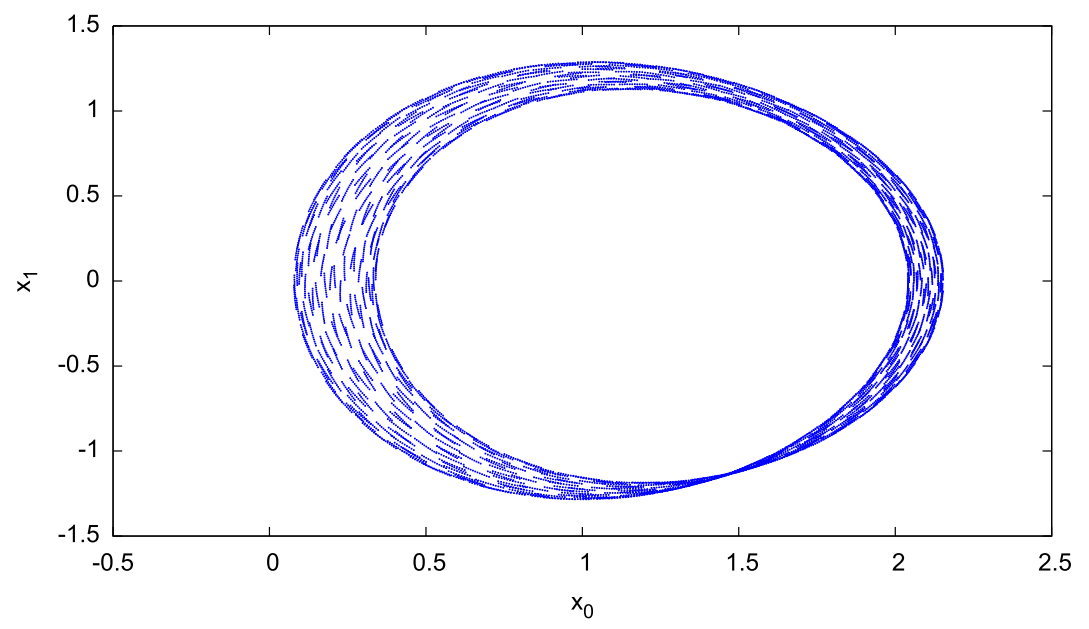

(a)

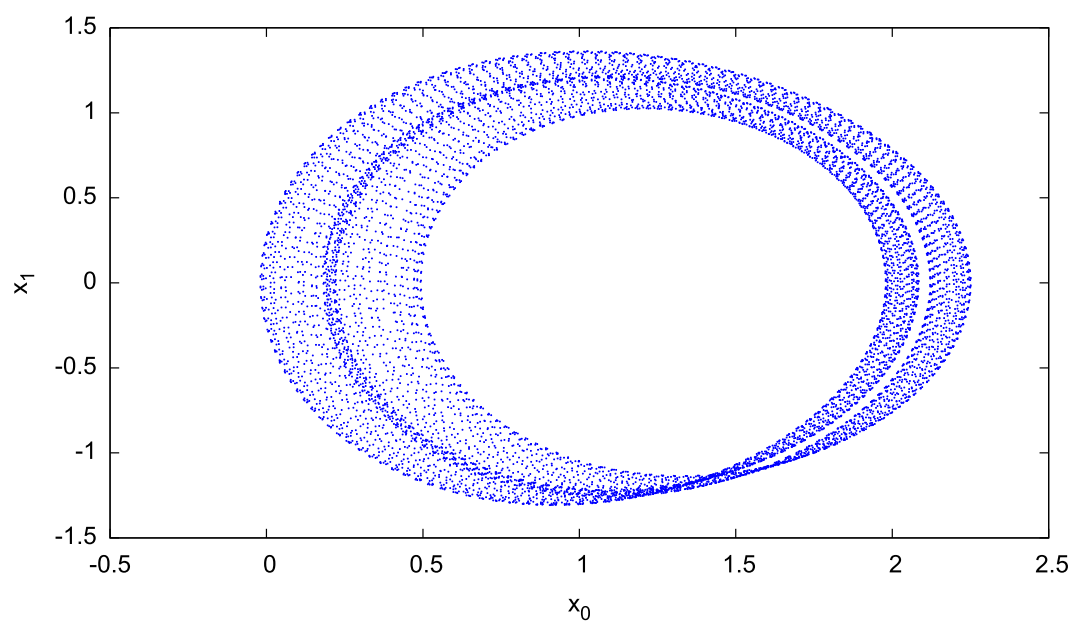

(b)

Fig. 29. MT2 on the section $\Sigma_{1}=\left\{x_{4}=0, \dot{x}_{4}>0\right\}$. (a) Before the FT3DC at $\omega_{1}=1.284$ and (b) after the FT3DC at $\omega_{1}=1.278$.

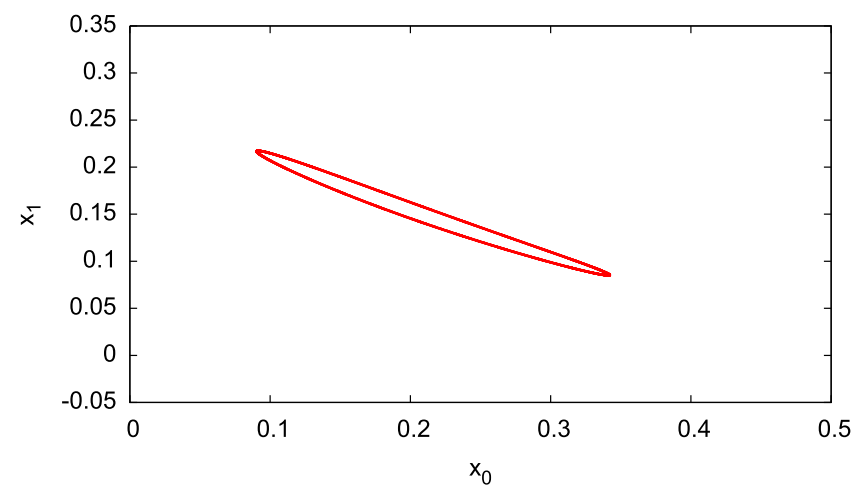

(a)

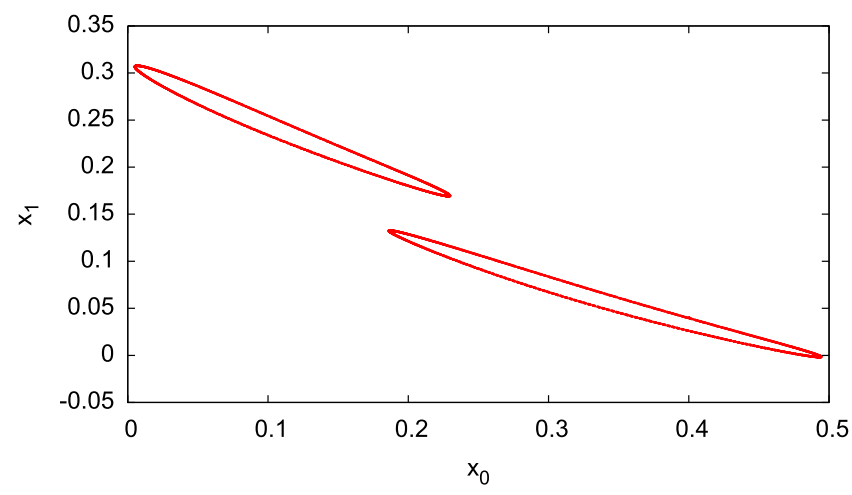

(b)

Fig. 30. ST1 on the section $\Sigma_{2}=\left\{x_{2}=0, \dot{x}_{2}>0, x_{4}=0\right\}$. (a) Before the FT3DC at $\omega_{1}=1.284$ and (b) after the FT3DC at $\omega_{1}=1.278$. 


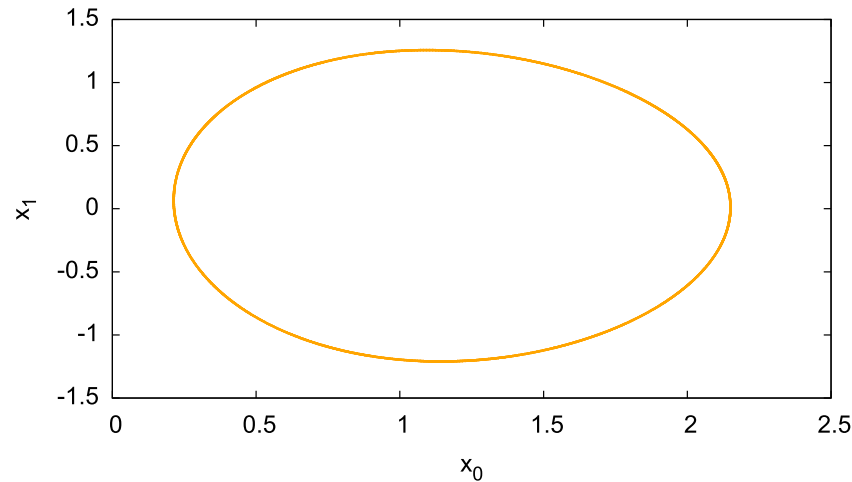

(a)

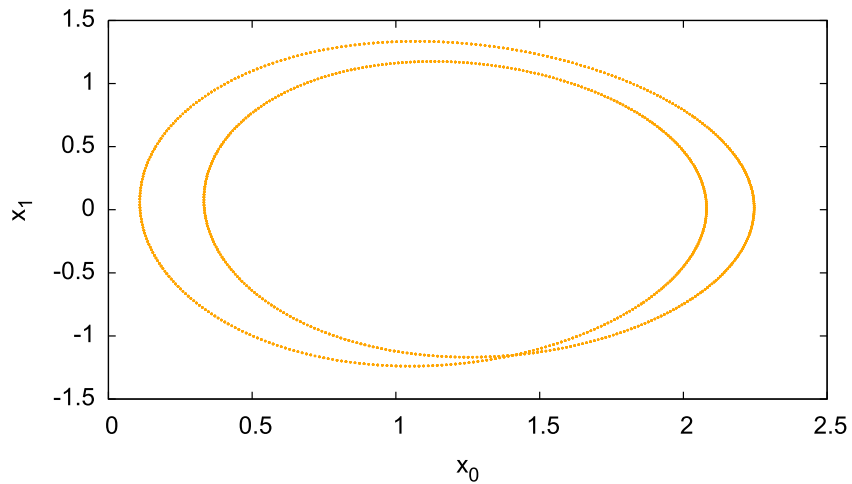

(b)

Fig. 31. ST1 on the section $\Sigma_{3}=\left\{x_{3}=0, \dot{x}_{3}>0, x_{4}=0\right\}$. (a) Before the FT3DC at $\omega_{1}=1.284$ and (b) after the FT3DC at $\omega_{1}=1.278$.

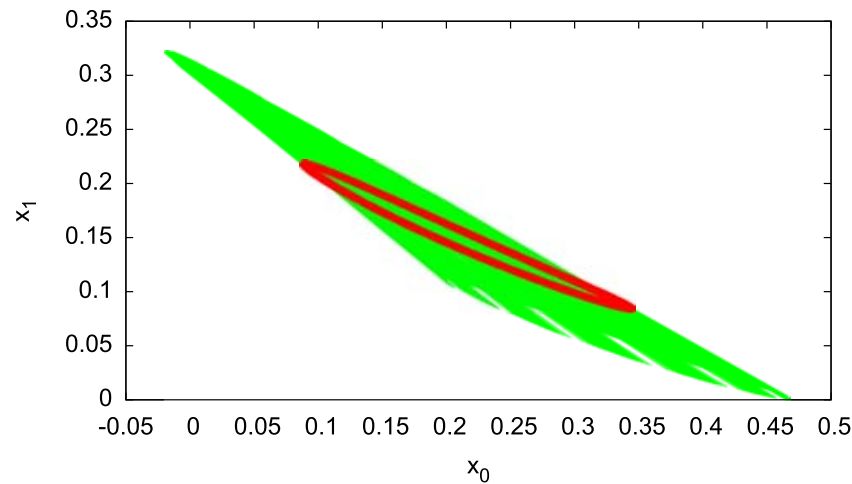

(a)

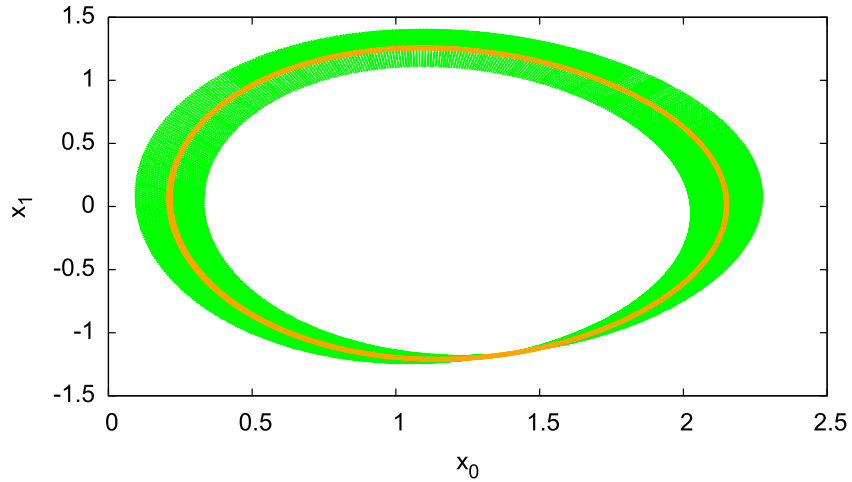

(b)

Fig. 32. A type $A^{*}$ and type $M$ DLBs of the ST1 before the FT3DC at $\omega_{1}=1.284$. (a) A type $A^{*}$ DLB on $\Sigma_{2}$ and (b) a type $M$ DLB on $\Sigma_{3}$.

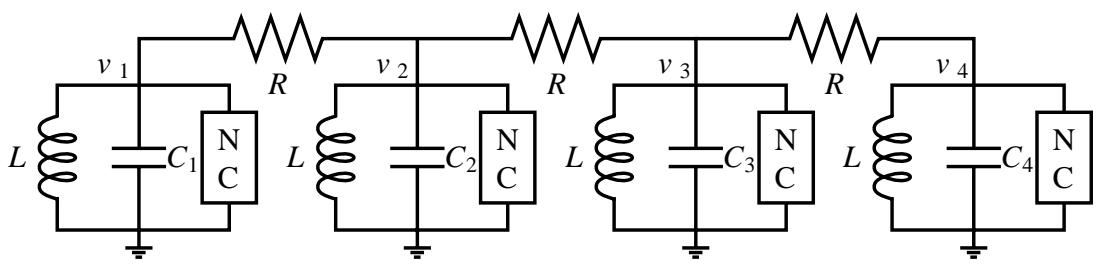

Fig. 33. The ladder of four-coupled VDP oscillators with large frequency detuning. 
The normalized ODE is given as follows:

$$
\left\{\begin{array}{l}
\dot{x}_{0}=x_{1} \\
\dot{x}_{1}=-\epsilon\left(b_{1}^{2}-x_{0}^{2}\right) x_{1}-x_{0}-\alpha\left(x_{1}-x_{3}\right) \\
\dot{x}_{2}=x_{3} \\
\frac{\dot{x}_{3}}{k_{2}^{2}}=-\epsilon\left(b_{2}^{2}-x_{2}^{2}\right) x_{3}-x_{2}-\alpha\left(2 x_{3}-x_{1}-x_{5}\right), \\
\dot{x}_{4}=x_{5} \\
\frac{\dot{x}_{5}}{k_{3}^{2}}=-\epsilon\left(b_{3}^{2}-x_{4}^{2}\right) x_{5}-x_{4}-\alpha\left(2 x_{5}-x_{3}-x_{7}\right), \\
\dot{x}_{6}=x_{7} \\
\frac{\dot{x}_{7}}{k_{4}^{2}}=-\epsilon\left(b_{4}^{2}-x_{6}^{2}\right) x_{7}-x_{6}-\alpha\left(x_{7}-x_{5}\right)
\end{array}\right.
$$

where $x_{0}, x_{2}, x_{4}$, and $x_{6}$ denote each oscillator's output, and $x_{1}, x_{3}, x_{5}$, and $x_{7}$ denote their derivatives, respectively. The parameter $\alpha$ denotes the coupling strength with $0 \leq \alpha \leq 1$, and $\epsilon$ denotes the degree of nonlinearity with $\epsilon \geq 0$. The parameter $k_{i}=C_{1} / C_{i}(i=2,3,4)$ denotes the frequency of oscillator $i$ relative to that of the first oscillator, ${ }^{19}$ and $b_{i}(i=1,2,3,4)$ specifies the amplitude of oscillator $i$. In the following calculation, the bifurcation parameter is $\alpha$, and the other parameters are fixed as $\epsilon=0.1, k_{2}=1.21, k_{3}=1.531, k_{4}=1.679$, and $b_{1}=b_{2}=b_{3}=b_{4}=1$. Figure 34 represents a oneparameter Lyapunov diagram showing FT3NS in
Eqs. (23). The multiplicity of the zero LE increases from two to four as the parameter $\alpha$ decreases. We conjecture that FT3NS bifurcation occurs at $\alpha=0.0426$, because the multiplicity of the zero LE changes from three to four and the DLE touches zero with multiplicity two at $\alpha=0.0426$. Figure 35 shows the DLB of ST1 on $\Sigma=\left\{x_{1}=0, \dot{x}_{1}<0, x_{4}=\right.$ $\left.0, x_{5}>0\right\}$ before the bifurcation at $\alpha=0.044$. The shape of the DLB is of type $F$. Figure 36 shows ST2 on $\Sigma$ after the bifurcation at $\alpha=0.042$. ST1 bifurcates to ST2 as a result of the bifurcation. Because the DLB of the $\mathrm{ST} 1$ is of type $F$, the original DLB of FT3 is of type $F^{3}$. Therefore, an FT3NS bifurcation occurs at $\alpha=0.0426$ in Fig. 34 .

\subsection{Saddle-node cycle bifurcation of FT3 (FT3SNC)}

In this subsection, as an example of FT3SNC, we analyze the same system as Eqs. (22). In this system, FT3SNC of the Type 2 solution is caused by phase locking between the intrinsic frequency of the Type 2 solution and some of the periodic signals. In the following calculation, the bifurcation parameter is $\omega_{1}$, and the other parameters are fixed as $\beta=0.56, \sigma=1.28, m_{1}=0.05, m_{2}=0.03$, $m_{3}=0.01, \omega_{2}=0.33123$, and $\omega_{3}=0.21123$. Figure 37 presents a one-parameter Lyapunov diagram of Eqs. (22). We observe a large frequency-locking region between $\omega_{1}=0.87$ and $\omega_{1}=1.05$. We focus

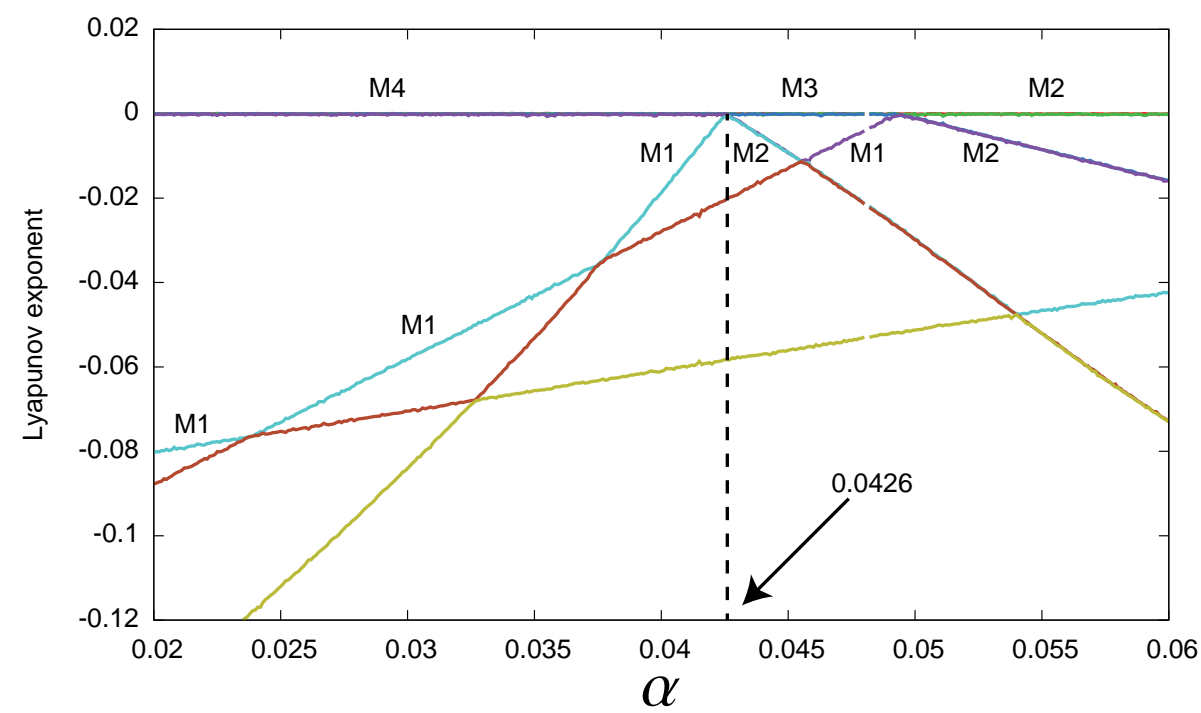

Fig. 34. One-parameter Lyapunov diagram, in terms of $\alpha$, obtained from Eq. (23). FT3NS is demonstrated at the point where the DLE touches zero at $\alpha=0.0426$.

\footnotetext{
${ }^{19}$ If the first oscillator frequency is $f_{1}$, then the second oscillator frequency is $f_{1} / k_{2}$.
} 


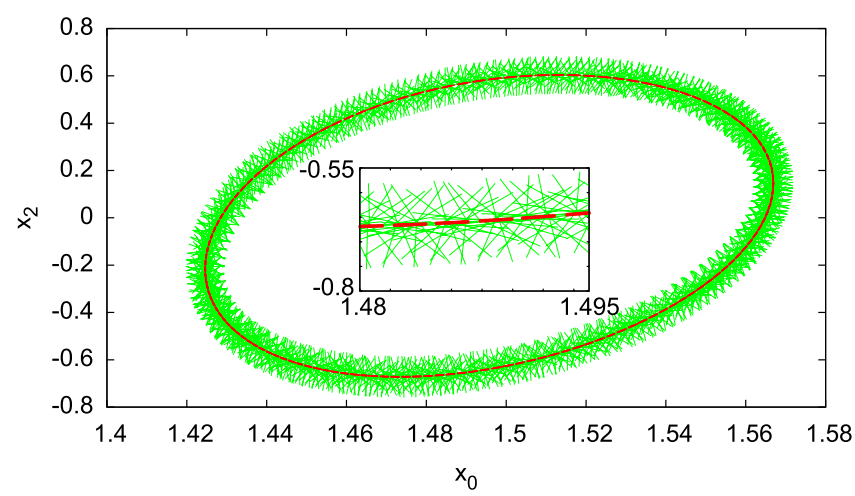

Fig. 35. The type $F$ DLB of ST1 on $\Sigma=\left\{x_{1}=0, \dot{x}_{1}<\right.$ $\left.0, x_{4}=0, x_{5}>0\right\}$ before the FT3NS at $\alpha=0.044$ in Eq. (23).

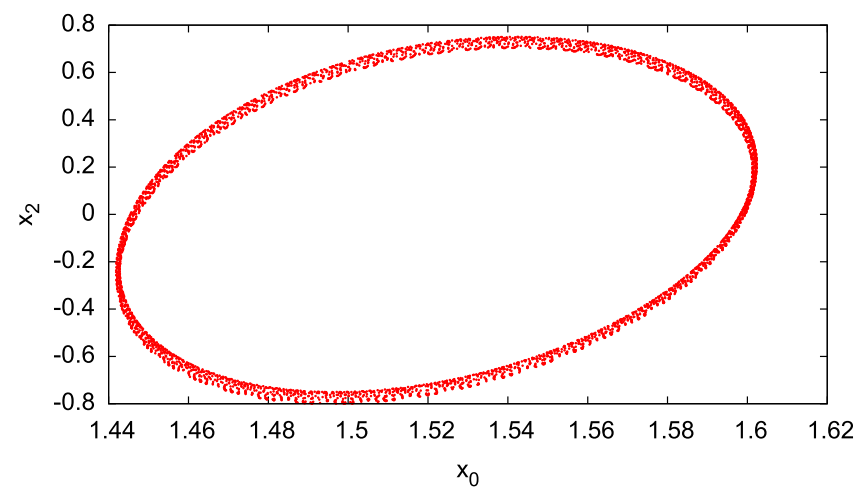

Fig. 36. ST2 on $\Sigma=\left\{x_{1}=0, \dot{x}_{1}<0, x_{4}=0, x_{5}>0\right\}$ after the FT3NS at $\alpha=0.042$ in Eqs. (23).

on this locking region, and calculate MT2, ST1, and DLB of the ST1 around the bifurcation point at $\omega_{1}=1.05$. Figure 38 shows the section tori on $\Sigma_{2}=\left\{x_{2}=0, \dot{x}_{2}>0, x_{4}=0\right\}$ before (red) and after (violet) the bifurcation, at $\omega_{1}=1.03$ and $\omega_{1}=1.0561$, respectively. From Fig. 38, we can infer that ST1 bifurcates to ST2 via the ST1SNC bifurcation. Figure 39 shows the DLB of ST1 on $\Sigma_{1}$ before

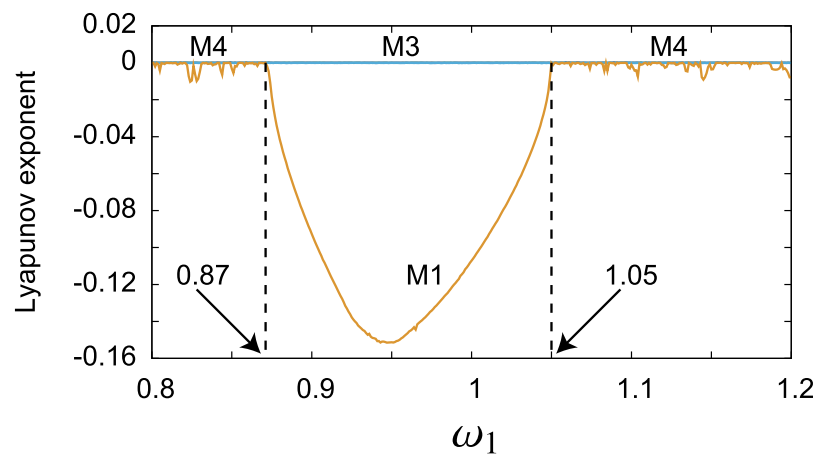

Fig. 37. One-parameter Lyapunov diagram of the Type 2 solution in terms of $\omega_{1}$, demonstrating FT3SNC at $\omega_{1}=1.05$ in the PLL circuit equations (22).

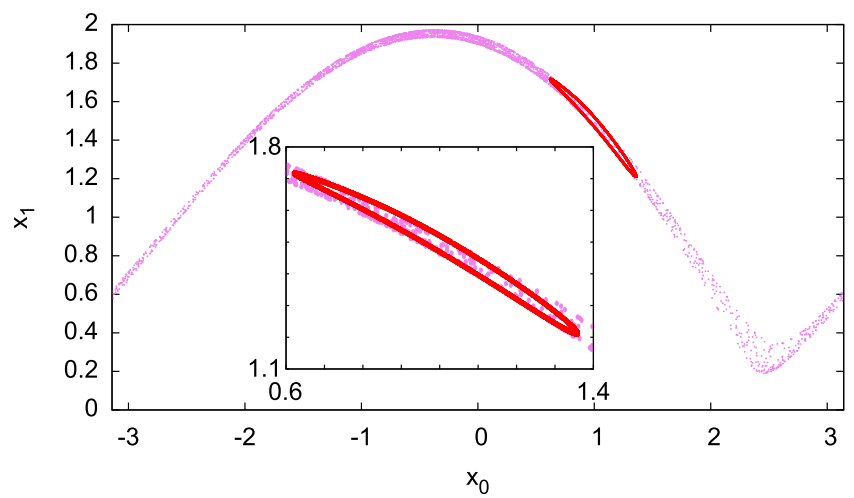

Fig. 38. The superimposed section-tori for the Type 2 solution on $\Sigma_{2}=\left\{x_{2}=0, \dot{x}_{2}>0, x_{4}=0\right\}$ in Eqs. (22) before (red) and after (violet) the FT3SNC bifurcation, at $\omega_{1}=1.03$ and $\omega_{1}=1.0561$, respectively. Note that the attractor before the bifurcation (red) is on the attractor after the bifurcation (violet). The region in the square is a magnified diagram for the attractors.

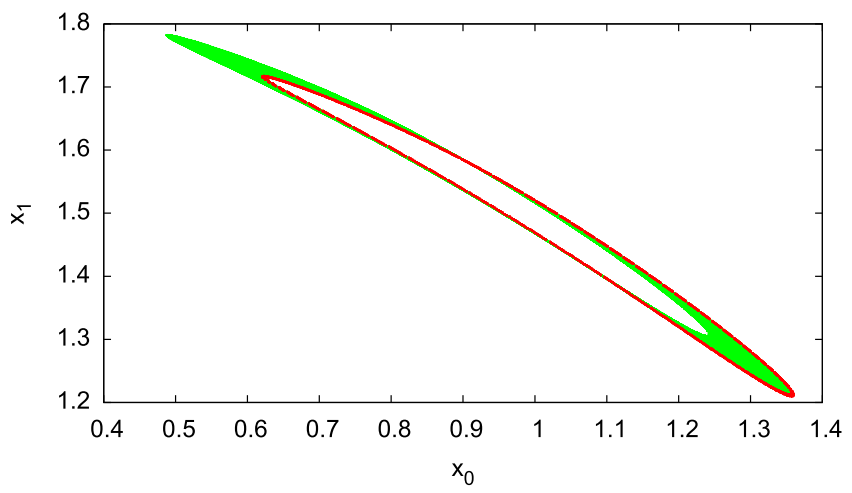

Fig. 39. A type $A^{+}$DLB of ST1 for the Type 2 solution on $\Sigma_{2}=\left\{x_{2}=0, \dot{x}_{2}>0, x_{4}=0\right\}$ before the FT3SNC at $\omega_{1}=1.03$ in Eqs. (22).

the bifurcation at $\omega_{1}=1.03$. The DLB of ST1 is of type $A^{+}$, hence the original DLB of the FT3 is of type $A^{3+}$. This bifurcation is FT3SNC because (1) the multiplicity of the zero LE changes from three to four in Fig. 37; (2) the ST1 before the bifurcation is embedded in the ST2 after the bifurcation in Fig. 38; and (3) the DLB in Fig. 39 is of type $A^{+}$.

\section{Conclusion}

We have classified the local bifurcations of $d$ dimensional tori in maps (MTd) and flows (FTd) for $d \geq 1$. First, we classified the dominant Lyapunov bundles (DLBs) of MTd and FTd. The DLBs of MTd can be classified into four types; namely, types $A^{d+}, A^{d-}, M^{d}$, and $F^{d}$. Furthermore, we presented a normal bifurcation conjecture for MTd; 
namely, a type $A^{d+}$ DLB causes a saddle-node bifurcation (MTdSN), a type $A^{d-}$ DLB causes a perioddoubling bifurcation (MTdPD), a type $M^{d} \mathrm{DLB}$ causes a double covering bifurcation (MTdDC), and a type $F^{d}$ DLB causes a Neimark-Sacker bifurcation (MTdNS). In addition, the DLBs of FTd can be classified in three types; that is, types $A^{d+}, M^{d}$, and $F^{d}$. We also presented a normal bifurcation conjecture for FTd; namely, a type $A^{d+}$ DLB causes a saddle-node bifurcation (FTdSN), a type $M^{d}$ DLB causes a double covering bifurcation (FTdDC), and a type $F^{d}$ DLB causes a Neimark-Sacker bifurcation (FTdNS). We next classified the resonance bifurcations of $\mathrm{MT} d / \mathrm{FT} d$ into two classes: saddlenode cycle $(\mathrm{MT}(d-1) \mathrm{SNC} / \mathrm{FT}(d-1) \mathrm{SNC})$ and heteroclinic cycle $(\mathrm{MT}(d-1) \mathrm{HCC} / \mathrm{FT}(d-1) \mathrm{HCC})$ bifurcations of the $(d-1)$-dimensional tori. The former is a reversible bifurcation, while the latter is an irreversible bifurcation with a hysteresis. The resonance bifurcations of MTd/FTd are represented by a type of global bifurcations of $(d-1)$-dimensional tori that emerge through heteroclinic connections of stable and unstable sets.

We have proposed a method for analyzing bifurcations of MTd/FTd that uses one-dimensional tori in sections (ST1) and zero-dimensional tori in sections (ST0). The bifurcations of ST1 can be classified into five classes: saddlenode (ST1SN), period doubling (ST1PD), component doubling (ST1CD), double covering (ST1DC), and Neimark-Sacker (ST1NS) bifurcations. The bifurcations of ST0 can be classified into four classes: saddle-node (STOSN), period doubling (STOPD), component doubling (ST0CD), and Neimark-Sacker (STONS) bifurcations. We presented a conjecture that MTdSN/FTdSN occurs if ST1SN/ST0SN is observed, MTdPD occurs if $\mathrm{ST1PD} / \mathrm{ST} 0 \mathrm{PD}$ is observed, $\mathrm{MT} d \mathrm{DC} / \mathrm{FT} d \mathrm{DC}$ occurs if ST1CD/ST1DC/ST0CD is observed, and MTdNS/FTdNS occurs if ST1NS/STONS is observed. We further presented typical examples for these bifurcations, both for maps and flows.

\section{Acknowledgments}

The work of second author (K. Kamiyama) was partially supported by Grant-in-Aid for Young Scientists (KAKENHI) (B) No. 50738383. The work of the last author (K. Aihara) was partially funded by ImPACT Program of Council for Science, Technology and Innovation (Cabinet Office, Government of Japan), and CREST, JST. The work of the first and last authors (M. Komuro and K. Aihara) was supported by the Aihara Project, the FIRST program from JSPS, which was initiated by CSTP.

\section{References}

Broer, H., Simo, C. \& Vitolo, R. [2008] "Hopf saddle-node bifurcation for fixed points of $3 \mathrm{~d}$ diffeomorphisms: Analysis of a resonance 'bubble'," Physica D 237, 1773-1799.

Broer, H. W., Huitema, G. B. \& Sevryuk, M. B. [1996] Quasi-Periodic Motions in Families of Dynamical Systems, Lecture Notes in Mathematics, Vol. 1645 (Springer-Verlag).

Endo, T. [1990] "Homoclinic orbits, fractal basin boundaries and bifurcations of phase-locked loop circuits," IEICE Trans. E 73, 828-835.

Kamiyama, K., Komuro, M., Endo, T. \& Aihara, K. [2014] "Classification of bifurcations of quasi-periodic solutions using Lyapunov bundles," Int. J. Bifurcation and Chaos 24, 1430034-1-30.

Kuznetsov, Y. [2004] Elements of Applied Bifurcation Theory, Applied Mathematical Sciences, Vol. 112, 3rd edition (Springer-Verlag).

Oseledets, V. I. [1968] "Multiplicative ergodic theorem: Characteristic Lyapunov exponents of dynamical systems," Trudy MMO 19, 179-210.

Ruelle, D. [1979] "Ergodic theory of differentiable dynamical systems," IHES Publ. Math. 50, 27-58.

Ruelle, D. \& Takens, F. [1971] "On the nature of turbulence," Comm. Math. Phys. 20, 167-192, http:// projecteuclid.org/euclid.cmp/1103857186. 